WING DEFLECTION ANALYSIS OF 3D PRINTED WIND TUNNEL MODELS

\author{
A Thesis \\ presented to \\ the Faculty of California Polytechnic State University, \\ San Luis Obispo
}

\author{
In Partial Fulfillment \\ of the Requirements for the Degree \\ Master of Science in Aerospace Engineering
}

by
Matthew Gerald Paul

June 2017 
(C) 2017

Matthew Gerald Paul

ALL RIGHTS RESERVED 
COMMITTEE MEMBERSHIP

TITLE: Wing Deflection Analysis of 3D Printed Wind Tunnel Models

AUTHOR: Matthew Gerald Paul

DATE SUBMITTED: June 2017

COMMITTEE CHAIR: Graham Doig, Ph.D.

Associate Professor of Aerospace Engineering

COMMITTEE MEMBER: Aaron Drake, Ph.D.

Associate Professor of Aerospace Engineering

COMMITTEE MEMBER: $\quad$ Eric Kasper, Ph.D.

Professor of Civil Engineering

COMMITTEE MEMBER: Kurt Long, M. S.

Laboratory Manager, NASA Ames Fluid

Mechanics Laboratory 


\section{ABSTRACT \\ Wing Deflection Analysis of 3D Printed Wind Tunnel Models}

This work investigates the feasibility of producing small scale, low aerodynamic loading wind tunnel models, using FDM 3D printing methods, that are both structurally and aerodynamically representative in the wind tunnel. To verify the applicability of this approach, a $2.07 \%$ scale model of the NASA CRM was produced, whose wings were manufacturing using a Finite Deposition Modeling 3D printer. Experimental data was compared to numerical simulations to determine percent difference in wake distribution and wingtip deflection for multiple configurations.

Numerical simulation data taken in the form of CFD and FEA was used to validate data taken in the wind tunnel experiments. The experiment utilized a wake rake to measure 3 different spanwise locations of the wing for aerodynamic data, and a videogrammetry method was used to measure the deflection of the wingtips for structural data. Both numerical simulations and experiments were evaluated at Reynolds numbers of 258,000 and 362,000 at 0 degrees angle of attack, and 258,000 at 5 degrees angle of attack.

Results indicate that the wing wake minimum in the wind tunnel test had shifted approximately $8.8 \mathrm{~mm}$ at the wingtip for the Nylon 910 wing at 258,000 Reynolds number for 0 degrees angle of attack when compared to CFD. Videogrammetry results indicate that the wing deflected 5.9mm, and has an $18.6 \%$ difference from observed deflection in FEA. This reveals the potential for small scale wind tunnel models to be more representative of true flight behavior for low loading scenarios. 


\section{ACKNOWLEDGMENTS}

I would like to start by thanking my advisor, Dr. Graham Doig, for not only aiding me in this project, but also being proactive in ensuring my success, both inside and outside of this research. I likely would not have gotten as far as I have in the aerospace industry without your tutelage and guidance, and certainly would not have the love for wind tunnel testing that I now have by simply working with you on projects. It has been an honor to be your graduate student, and I wish nothing but the best for you and your future projects in the Cal Poly Aerospace Department.

I am eternally grateful for my girlfriend Christina, for always being there when I needed another leg to stand on, and not being afraid to force me to focus on my work. You were there when I spent the long hard weeks of data analysis in front of a computer to reassure me and help me on my way, and I will never forget it.

To my family and friends who supported me and kept me in their thoughts, even when

I severed contact from almost everyone. Your support through this project was invaluable and always there when I needed it. 


\section{TABLE OF CONTENTS}

Page

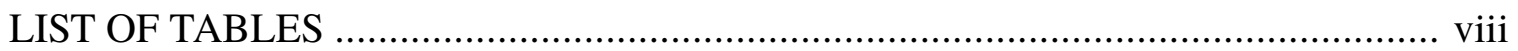

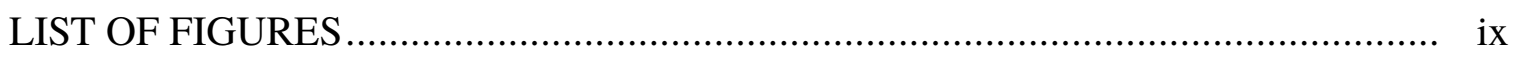

CHAPTER

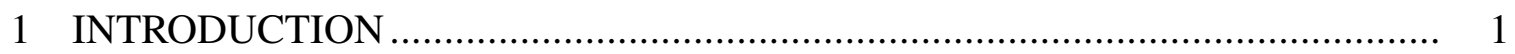

1.1 Wind Tunnel Experiments and Numerical Simulations ......................... 1

1.2 Motivations for Investigation............................................................ 2

1.3 State of the Art ......................................................................... 3

1.3.1 Elastic Wind Tunnel Models ................................................. 3

1.3.2 Coupled Numerical Simulations ......................................... 6

1.3.3 Additive Manufacturing...................................................... 6

1.4 Methodology of this Work …………………………..................... 8

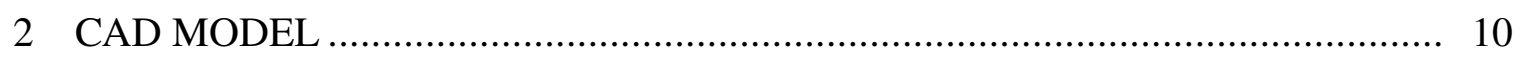

2.1 Configuration Metrics .................................................................. 10

2.2 Manufacturing Model ................................................................... 11

2.2.1 Wing Assembly ............................................................ 11

2.2.2 Fuselage ……........................................................... 13

2.2.3 Tunnel Mounting ............................................................. 17

2.3 CFD Fluid Domain ................................................................... 19

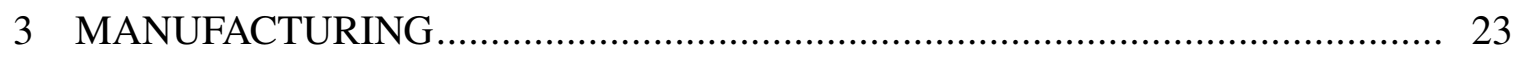

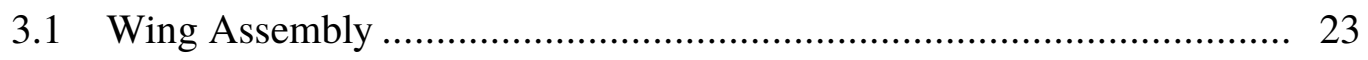

3.1.1 3D Printing ................................................................. 24

3.1.2 Post Processing and Assembly …..................................... 27

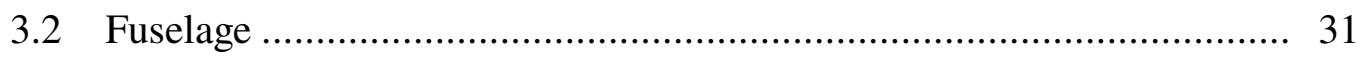


3.3 Wall Mounting ................................................................................. 32

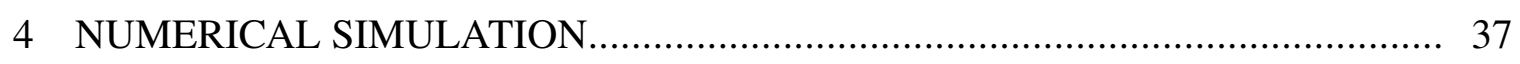

4.1 Computational Fluid Dynamics........................................................... 37

4.1.1 Meshing ...................................................................... 37

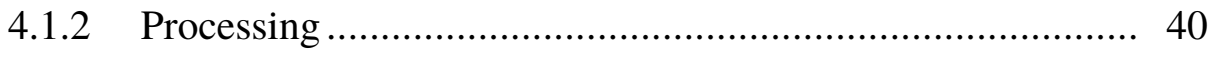

4.2 Finite Element Analysis ...................................................................... 44

4.2.1 Meshing …….............................................................. 45

4.2.2 Processing ...................................................................... 48

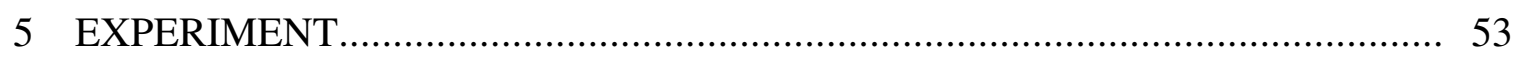

$5.1 \quad$ Setup and Preliminary Testing …………………………………... 53

$5.2 \quad$ Wake Rake .................................................................................. 55

5.3 Videogrammetry ....................................................................... 57

5.3.1 Hardware Settings ...................................................... 57

5.3.2 Software Settings............................................................ 58

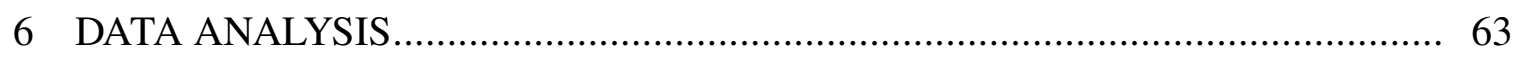

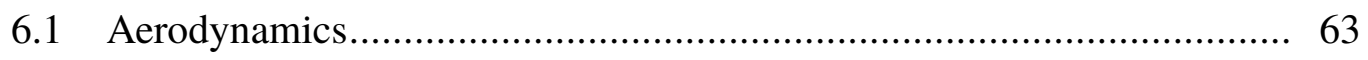

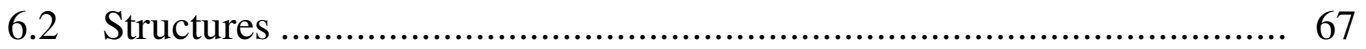

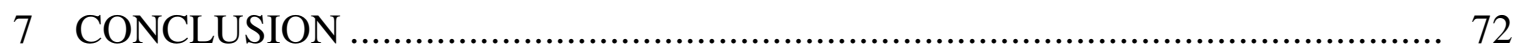

7.1 Future Work Considerations............................................................... 73

7.1.1 Experimental ............................................................ 73

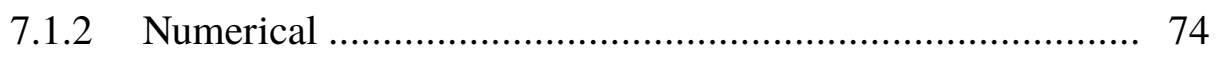

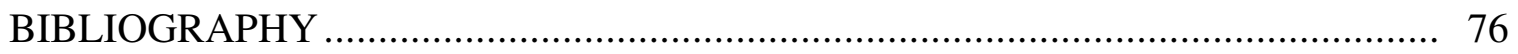

APPENDICES

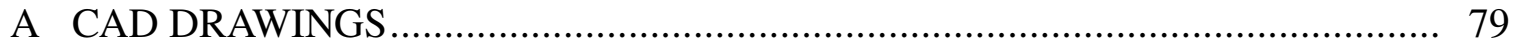

B MESHING PARAMETERS ……………................................................... 84 


\section{LIST OF TABLES}

Table

3.1 Rapid prototyping materials for the CRM wing sections ${ }^{[12]}$, ${ }^{[14]}$

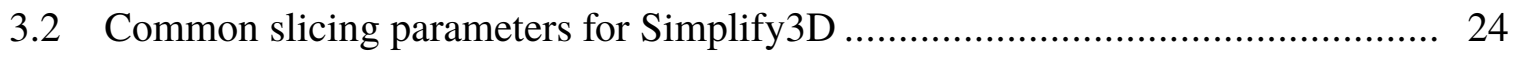

3.3 Varied printing parameters for Simplify3D .............................................. 25

4.1 Mesh statistics for both modeled cases .................................................. 40

4.2 Mesh convergence for the $25 \mathrm{~m} / \mathrm{s}, 0$ angle of attack configuration; percent difference based off deviation from finest mesh ......................................... 42

4.3 Mesh convergence for the 5 degrees angle of attack configuration; Percent difference based off deviation from finest mesh ....................................... 50

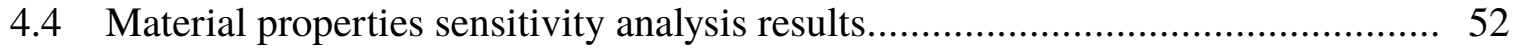

5.1 $\mathrm{Y}$ and $\mathrm{Z}$ axis locations of the traverse for the wake rake; Distances are measured

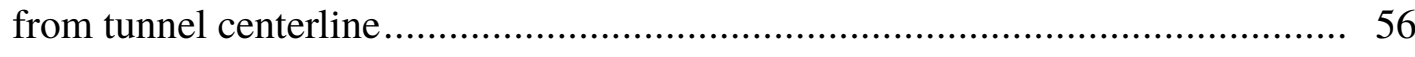

6.1 Change in $\mathrm{Z}$ location of wake peaks for each configuration when compared to CFD

6.2 Deflection results from the videogrammetry experiment performed in the Cal Poly Low Speed Wind Tunnel ................................................................. 69

6.3 Results of the FEA simulations compared to the videogrammetry experiment..... 70

B.1 Global meshing parameters for both angles of attack............................... 84

B.2 Global meshing parameters for the FEA simulation …............................. 84 


\section{LIST OF FIGURES}

Figure

Page

1.1 Depiction of wing geometry shapes based on loading scenario ${ }^{[3]} \ldots \ldots \ldots \ldots \ldots \ldots \ldots . . . . . . . . .3$

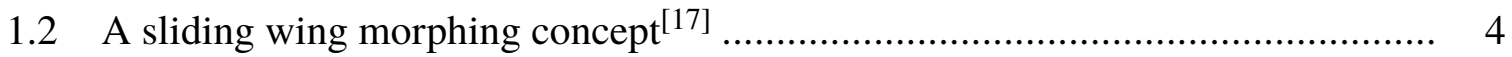

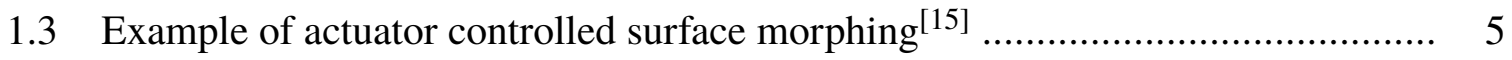

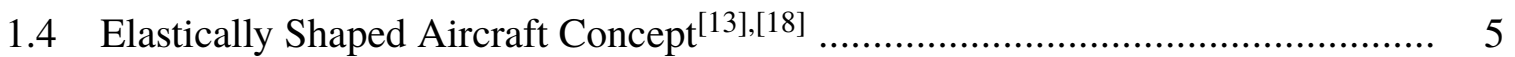

1.5 Example of surface roughness produced by sequential layers of $3 \mathrm{D}$ printing ${ }^{[8]} \ldots \quad 7$

1.6 NASA Common Research Model in the Ames Research Facility ${ }^{[16]}$.................. 9

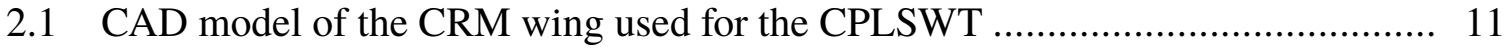

2.2 Depiction of extrusions made in the tipwing and midwing for slotting purposes.. 12

2.3 Wing assembly construction in Solidworks prior to printing ......................... 13

2.4 Scaled down fuselage CAD prior to OML editing ...................................... 14

2.5 Cavity extrusions produced for slotting the wing and tunnel block to the fuselage 14

2.6 Holes cut into the assembly to allow shear pin access and removal ................... 15

2.7 Extrusion added to fuselage to escape the tunnel wall boundary layer ................ 16

2.8 CAD of the tunnel mounting hardware to hold the model stable in the wind tunnel 17

2.9 See through schematic of the tunnel block and cylinder assembly .................... 18

2.10 Depiction of tunnel mounting assembly to test section wall ........................... 18

2.11 Depiction of tunnel mounting assembly to test section wall........................... 19

2.12 Test section fluid domain used for input into CFD meshing ........................... 20

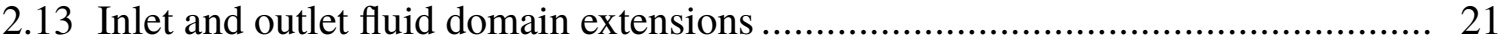

2.14 Laminar zones for CFD laminar to turbulent transition................................ 22

3.1 Simplify3D print preview example of the midwing at $100 \mathrm{~mm}$ above the print bed 26 
3.2 Example of parts after they have been printed .............................................. 28

3.3 Wing pieces after sanding and before bonding to showcase slotting method ........ 28

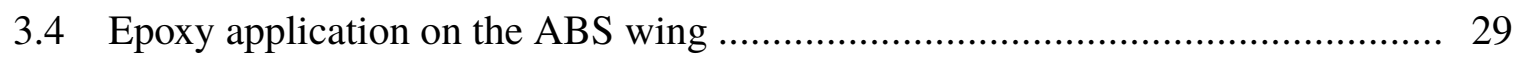

3.5 First coat of paint on the ABS wing .......................................................... 31

3.6 Fuselage sections after having primer applied to the wetted side ....................... 32

3.7 Final test ready model with the ABS wing ................................................... 33

3.8 Individual parts used in the tunnel mounting assembly .................................... 34

3.9 Mounting assembly prior to the attachment of the tunnel block .......................... 34

3.10 Tunnel block attachment with a digital level for precise angle of attack .............. 35

3.11 Wind tunnel model installed in the test section............................................... 36

4.1 Bodies used to specify area of increased mesh density in Ansys ........................ 38

4.2 Slices in mesh for observation of inflation layer generation in Ansys .................. 39

4.3 Region of cell refinement to improve cell skew and mesh quality ....................... 40

4.4 Final mesh views for the 0 degree angle of attack simulation............................. 40

4.5 Residual results for each Fluent case ......................................................... 41

4.6 Comparison of turbulence models for Low Reynolds number transition in Field-

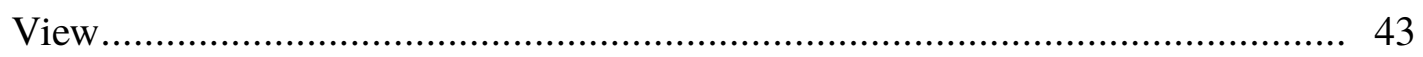

4.7 Example of contact region for the basewing to the midwing ............................. 46

4.8 Mesh views for the FEA simulation ............................................................ 47

4.9 Pressure loading vectors at each node for the $25 \frac{\mathrm{m}}{\mathrm{s}}$ loading case ........................ 48

4.10 Fixed support for all loading cases ........................................................... 49

4.11 Structured mesh for FEA element type sensitivity analysis ................................ 51

5.1 Tethering of the ABS wing for pre-test check loads ........................................... 54

5.2 Oil flow attachment and separation for Cal Poly CRM ...................................... 55

5.3 Various views of the 5-hole probe used in the wake study …............................. 57

5.4 View that each camera has of the wing configuration during testing ................... 59 
5.5 Dot tracking initialization in Matlab for 2 configurations .............................. 60

5.6 Dots being tracked by the $\mathrm{x}$-axis camera ............................................. 61

5.7 Screen captures of the dot tracking in Matlab during processing ...................... 62

6.1 Total pressure ratios and their vertical locations for all wings and configurations

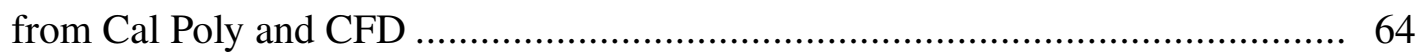

6.2 Pressure drop peak locations from tunnel centerline for both wings at $25 \mathrm{~m} / \mathrm{s} \ldots \ldots 65$

6.3 Wing wake comparison from the Cal Poly wake rake probe ............................ 66

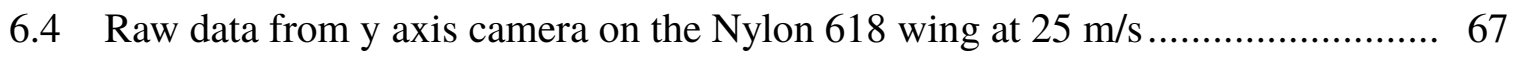

6.5 Clipped data from y axis camera on the Nylon 618 wing at $25 \mathrm{~m} / \mathrm{s} \ldots \ldots \ldots \ldots \ldots \ldots . . . . . .68$

6.6 Average data from y axis camera on the Nylon 618 wing at $25 \mathrm{~m} / \mathrm{s} \ldots \ldots \ldots \ldots \ldots \ldots \ldots . . . \ldots 8$

6.7 Undeformed and deformed wing result from FEA, with indication of probe

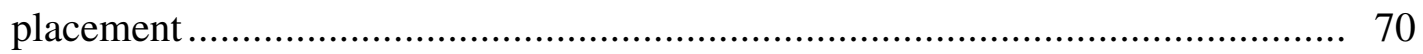

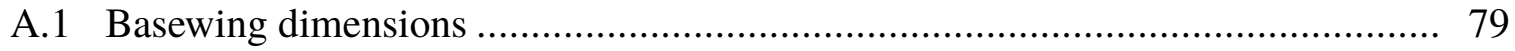

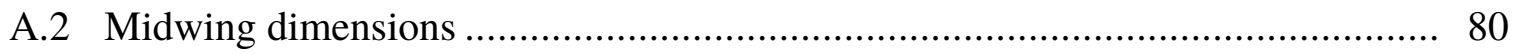

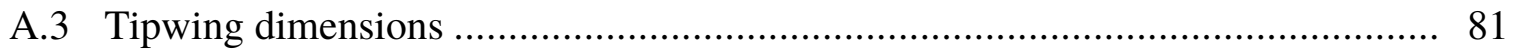

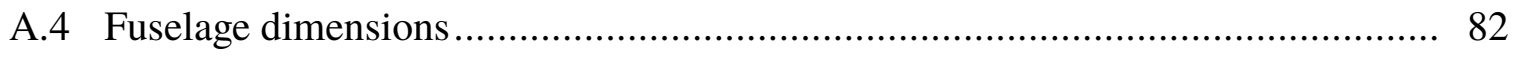

A.5 Tunnel mounting assembly dimensions ….......................................... 83 


\section{INTRODUCTION}

\subsection{Wind Tunnel Experiments and Numerical Simulations}

In the current stage of aerodynamic analysis, the wind tunnel model is still one of the most accurate methods of determining the performance of a body in a fluid. Wind tunnel testing is being used in an increasingly wider array of applications, varying from determining lift and drag characteristics of an aircraft to identifying chemical saturation content of pipe leak. From an industrial standpoint, wind tunnel testing is required for almost all aircraft to verify aerodynamic, structural, and control performance of that aircraft. These experiments are not only vital in terms of safety precautions, but also much cheaper, easier, and safer than producing a full-scale test flight article to obtain the information needed. This understanding has lead to ever more advanced testing techniques and concepts related to wind tunnel experiments to improve the quality and efficiency of the data obtained.

However, these advancements have lead to an increase in complexity of the test methods necessary to perform a wind tunnel experiment, as well as the cost and resources increase for various investigations. This has lead to the development and use of virtual simulations, such as Computational Fluid Dynamics (CFD) and Finite Element Analysis (FEA), to decrease the amount of time, money, and resources required to obtain the information needed for a particular study. Coupling information obtained from a wind tunnel model with numerical simulations of that model will eventually lead to an improved end user product, or at least an end product that is more accurate had wind tunnel testing been dismissed.

With the advancements in model manufacturing and numerical simulations available to the public, it is deemed possible to produce a small scale wind tunnel model whose deflection and aerodynamic phenomena are represented in a wind tunnel experiment for 
low loading scenarios. Through the same process used to produce a jig model, a user would be able to use CFD and FEA to determine the aerodynamics and model deflection present for multiple low Reynolds flow regimes and geometric orientations, and use 3D printing to alter the interior and exterior of that model such that it matches those numerical simulations.

\subsection{Motivations for Investigation}

There is room for improvement in regard to the design and manufacture of models for wind tunnel testing, specifically scale models of air vehicles for both commercial and military applications. Currently, models made for wind tunnel applications are primarily done via subtractive manufacturing, and are designed such that the models do not become elastically or plastically deformed under strong aerodynamic forces. This requires the models to be made from either high strength aluminum or steel to ensure the safe operation of wind tunnel equipment and preservation of the wind tunnel model. While practical, this method inhibits the model's ability to deform under such loads, leading to the potential for an inaccurate representation of that model in a real world scenario.

Industrial wind tunnel test engineers, aerodynamicists, and structural engineers alike are aware of the issue, and it is common practice to create a "jig model" to circumvent this issue ${ }^{[3],[9],[13],[18]}$. The jig model is created through a process of both aerodynamic and structural numerical simulations to determine the deflection the model should undergo for a particular loading test or Reynolds Number regime. Once a solution has converged, the deflected model shape is "subtracted" from the $1 \mathrm{~g}$ loaded shape to produce a geometry reminiscent of what the actual body should appear to be in a real life scenario, as seen in Figure 1.1. This geometry is then made in addition to the original wind tunnel model to provide more accurate aerodynamic data at the deflected body design point. 


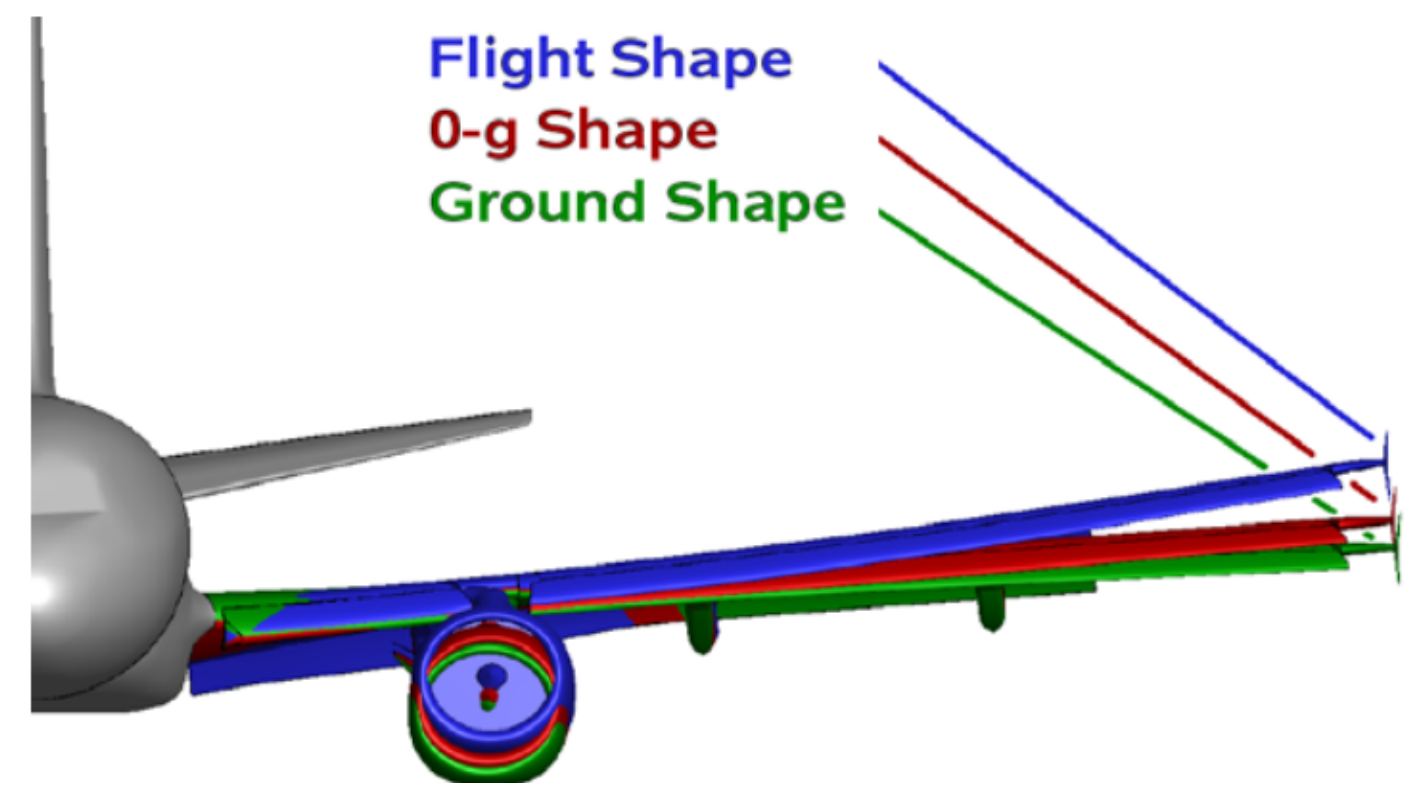

Figure 1.1: Depiction of wing geometry shapes based on loading scenario ${ }^{[3]}$

While this method is acceptable to obtain the data required in the investigation, it is not necessarily the most efficient. This method requires not only the procurement of many different geometric shapes, depending on the depth of the study, but also additional testing time of the model. This increases the resources necessary to obtain the information as well as the cost of that information.

\subsection{State of the Art}

\subsubsection{Elastic Wind Tunnel Models}

While the majority of wind tunnel models are made primarily not to deflect during testing, experiments have been done using elastic models for various purposes. One such method is to use a "surface morphing" method to actively or passively deflect the model during testing[13],[15],[17]. While the purpose of these models is usually intended to serve other aerodynamic purposes, such as seamless control surfaces or variable camber wings, it is occasionally employed to produce a deflection in the model that is present during some flow 
regime of the model. This concept could be employed to deflect a model according to the desires of the user based on actual flight data or CFD.

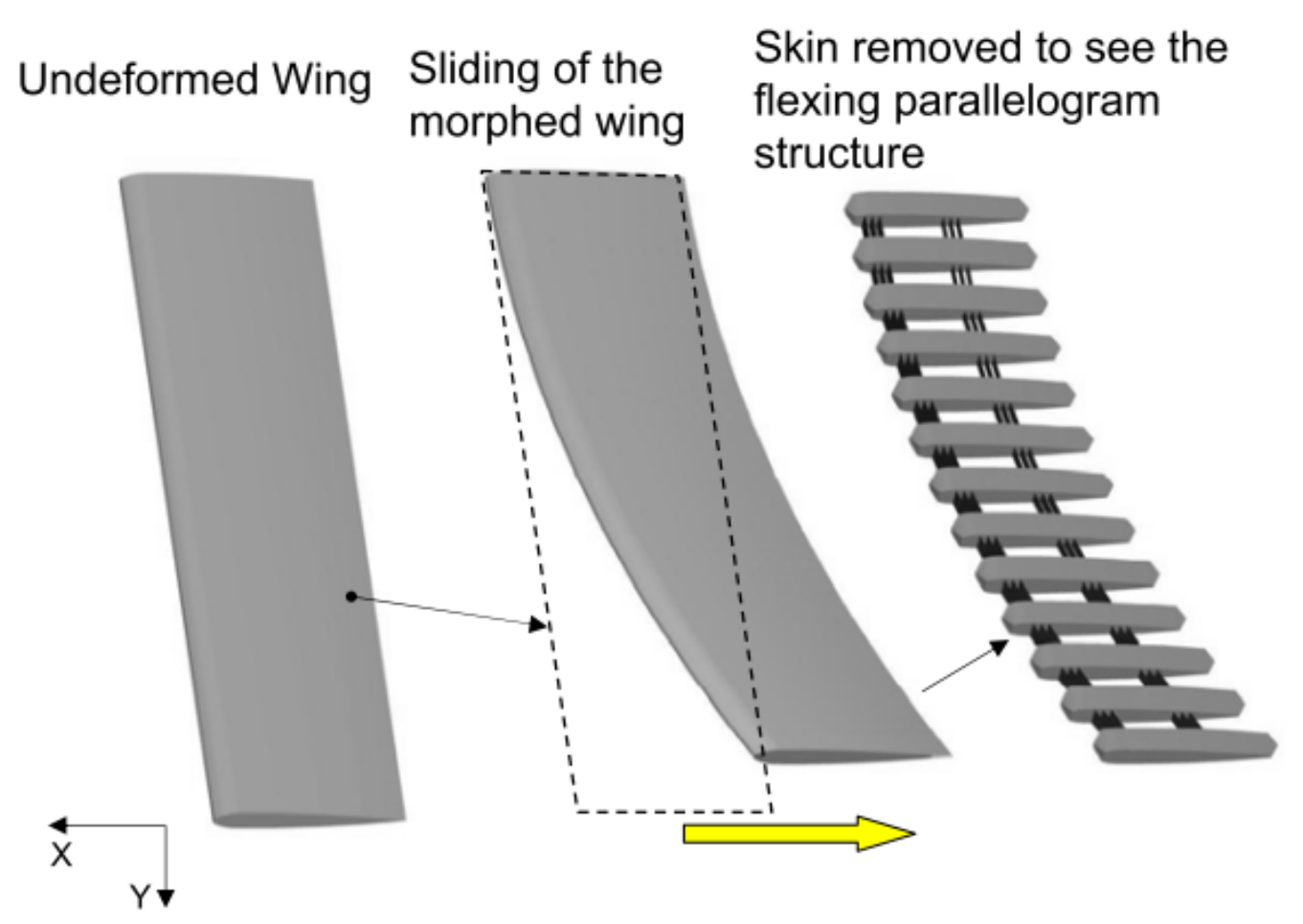

Figure 1.2: A sliding wing morphing concept ${ }^{[17]}$

However, this method has multiple drawbacks, the foremost being complexity. For active surface morphing, tools must be placed inside the skin of the wing and maintain the ability for user control outside of the model and tunnel. This is usually done using actuators or servos, seen in Figure 1.3, though can be done through other methods as well. Another drawback is availability and procurement of special materials. Certain alloys and materials have proven the ability to morph their shape without the need of complex mechanisms to control such activity. However, these materials are not always readily available, and can be expensive if they are.

NASA has made the beginning steps in the field of a static-aeroelastic wind tunnel model for its "Elastically Shaped Aircraft Concept (ESAC)", and is the most relevant work for this study ${ }^{[18]}$. NASA set out to explore whether it could predict the aerodynamics 


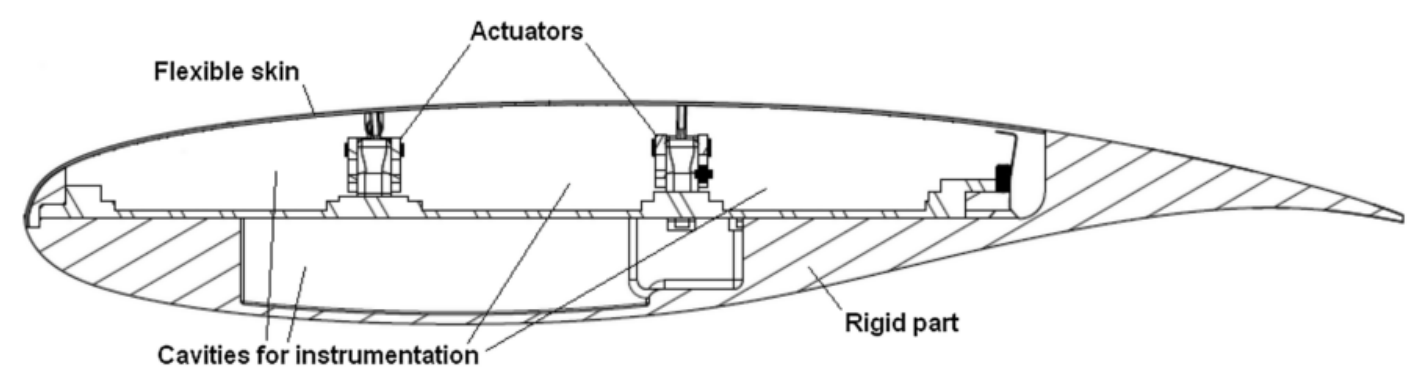

Figure 1.3: Example of actuator controlled surface morphing ${ }^{[15]}$

and structural shape of the Generic Transport Model with a 10\% wing deflection using numerical simulations, shown in Figure 1.4. This concept was then experimentally tested for verification of the prediction model ${ }^{[13]}$. While Nguyen used the ESAC to explore the merit of a continuous control surface, it was still shown that the prediction methods produced by Ting were in fact accurate. While many of the conclusions and procedures of these two works will be utilized in this study, it should be noted that this study differs in two ways. The first is that the hypothesis being explored here involves predicting the aeroelastic properties for multiple flow scenarios, rather than designing to a specific deflection point. The second is that this work focuses on using additive manufacturing methods, rather than subtractive manufacturing methods to produce the models in question.

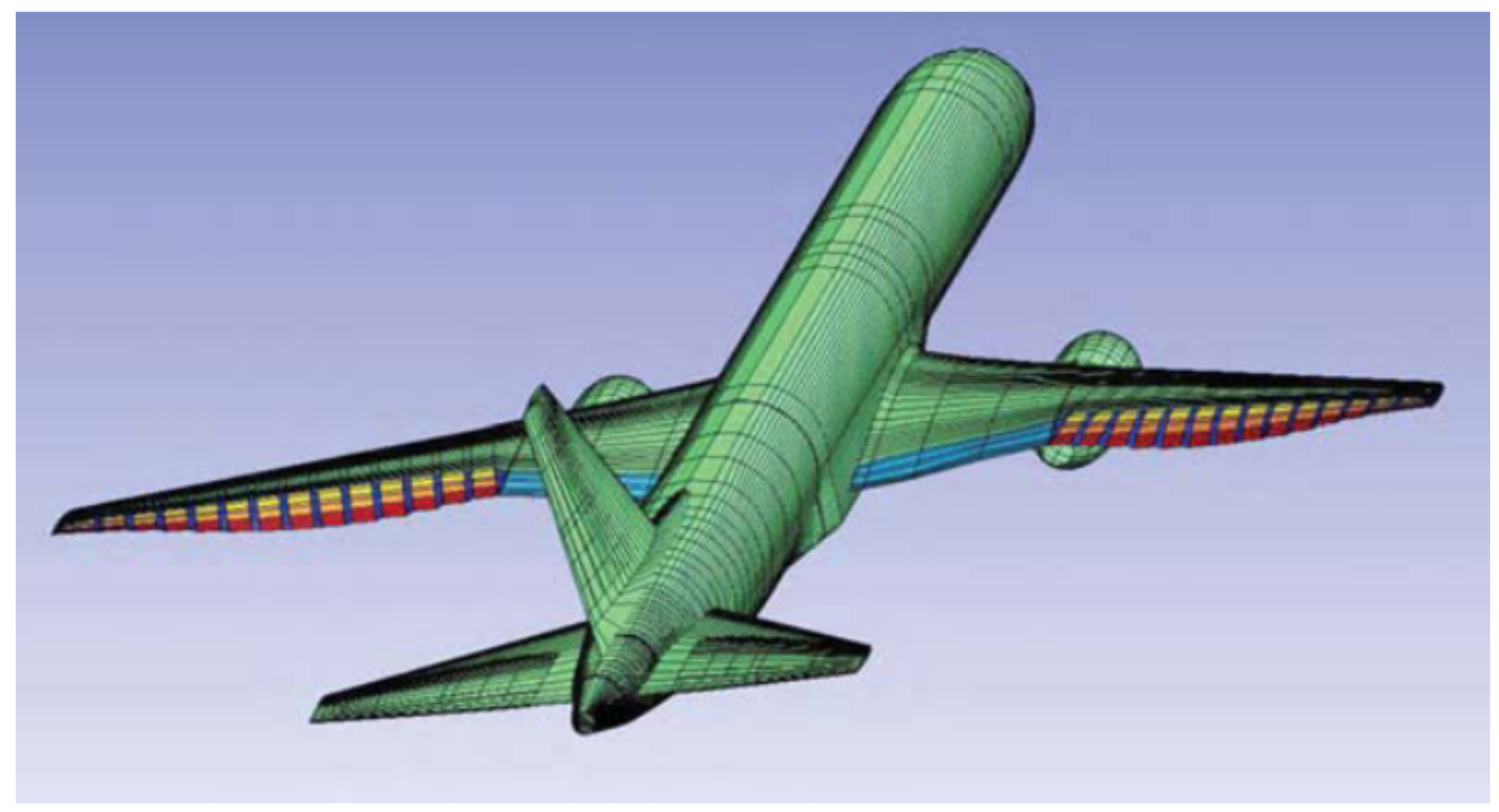

Figure 1.4: Elastically Shaped Aircraft Concept ${ }^{[13],[18]}$ 


\subsubsection{Coupled Numerical Simulations}

In order for jig models to made for wind tunnel testing, and for the purposes of this study, coupled numerical simulations utilizing both CFD and FEA are necessary to predict the outcome of experimental data. It is therefore imperative to ensure that these methods have proven correlation to observed phenomena, whether in a wind tunnel or flight test experiment, such that it can be used in this study. Airbus performed a fluid-structure interaction simulation to verify flight test data of the Airbus A340-300 using NASTRAN ${ }^{[10]}$. Results of this study proved the correct representation in the numerical simulations to the flight test data. A similar study was performed at transonic speeds by Hooker, who instead compared to wind tunnel model data rather than flight test data ${ }^{[9]}$. It was shown that using Navier-Stokes CFD methods, coupled with structural FEM, was sufficient to produce

jig models for wind tunnel testing. However, Hooker did go on to mention that further definitions of the chordwise model was necessary for a more accurate prediction. Finally, a more modern application of coupled numerical simulations was performed by Cella, who found good agreement between wind tunnel model data and predictions of commercially available structural and fluid dynamic codes ${ }^{[6]}$.

\subsubsection{Additive Manufacturing}

The process of producing parts via additive manufacturing, or 3D printing, is appealing primarily because of the relatively low cost of production, part lead time, ease of use, and overall user control of the end product. This is especially appealing for wind tunnel models that have complex geometries and are difficult or impossible to produce with subtractive manufacturing. Typically, 3D printed wind tunnel models are produced via stereolithography (SLA) as it is not only structurally stable, but can be manufactured to very low tolerance values. This is especially important from an aerodynamics perspective, where the surface 
roughness of a part has a significant effect on the flow field and subsequent data ${ }^{[8]}$. The issue here is that SLA printers and the material used by it can be costly, and therefore outside procurement means for many institutions. A much more cost effective method of rapid prototyping, and more widely used, is done through Finite Deposition Modeling (FDM). This method involves melting a filament spool of thermoplastic, and rebuilding it layer by layer based on a slicing profile of a model. However, is not typically used for professional wind tunnel tests on the grounds that it is not strong enough to withstand high aerodynamic loads and that its surface finish is not fine enough ${ }^{[7]}$. It should be noted that this surface roughness of FDM, illustrated by Figure 1.5, can be mitigated through post-processing of the models such as sanding or the use of paint.

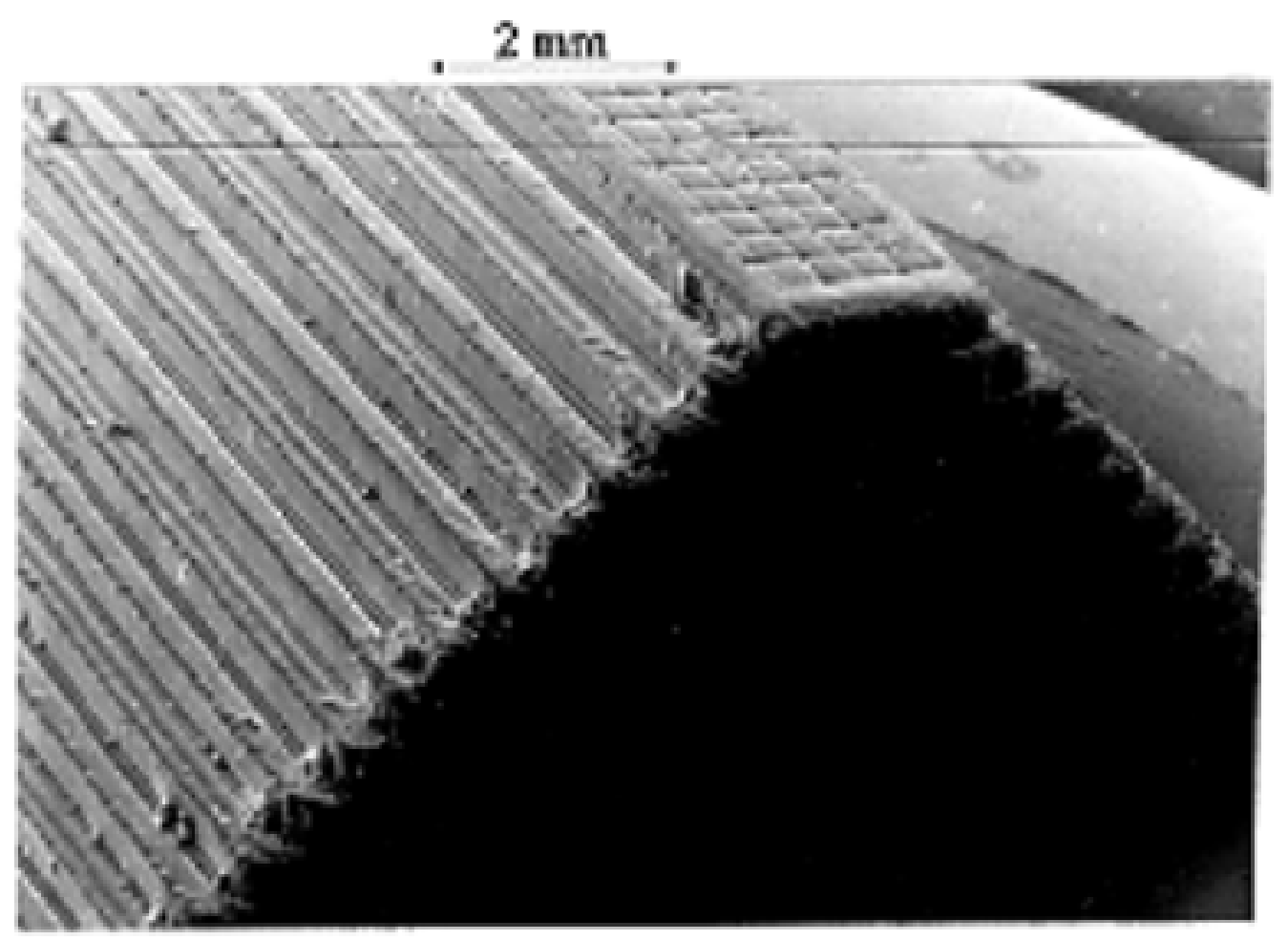

Figure 1.5: Example of surface roughness produced by sequential layers of $3 \mathrm{D}$ printing ${ }^{[8]}$

Structural integrity of FDM models is a much bigger concern for professional grade wind tunnel models due to the risk that these models impose on the test equipment. However, 
the ability of these models to be produced quickly and cheaply often mitigate these risks for some institutions. It is for these reasons that an FDM printer will be used for this study, on the grounds that the integrity of the experiment will be maintained as long as the model only deforms elastically, not plastically. Attempts to predict the elastic deflection of the model will be performed in FEA, and therefore will require material property data using ASTM standards ${ }^{[1],[2]}$. Investigations of the material properties of FDM parts have been

performed using commercially available printers and commonly used materials ${ }^{[19]}$.Due to the limitation of resources available for this project, material properties for each model printed will be obtained by prior experiments by the manufacturer ${ }^{[14],[12]}$

\subsection{Methodology of this Work}

This study will seek to investigate the prediction of both aerodynamic and structural data of a $2 \%$ scale wind tunnel model in multiple flow regimes using a 1-way, coupled analysis of CFD and FEA. The NASA Common Research Model (CRM) was chosen as the configuration to be tested due to its large library of available data, and its relevance in both the wind tunnel and numerical simulation communities. The wind tunnel model used by NASA, seen in figure (1.6), consists of a Wing, Body, Nacelle, and Pylon (WBNP). However, only the wing and body will be considered in the scope of this study.

To ensure predictability for low Reynolds flow regimes and loading scenarios, different configurations and materials of this model will be tested. The model will undergo testing Reynolds numbers of 258,000 and 362,000 at an angle of attack of 0 degrees, and 258,000 at 5 degrees. Furthermore, the wing will be produced using ABS, Nylon 618, and Nylon 910 printing material via FDM printing methods, and will be printed using a Lulzbot Taz 6 FDM printer. The combination of these configurations and manufacturing materials will produce a broad enough range to verify prediction of aerodynamic and structural phenomena using CFD and FEA codes within the ANSYS software suite. Experimental data will be acquired 


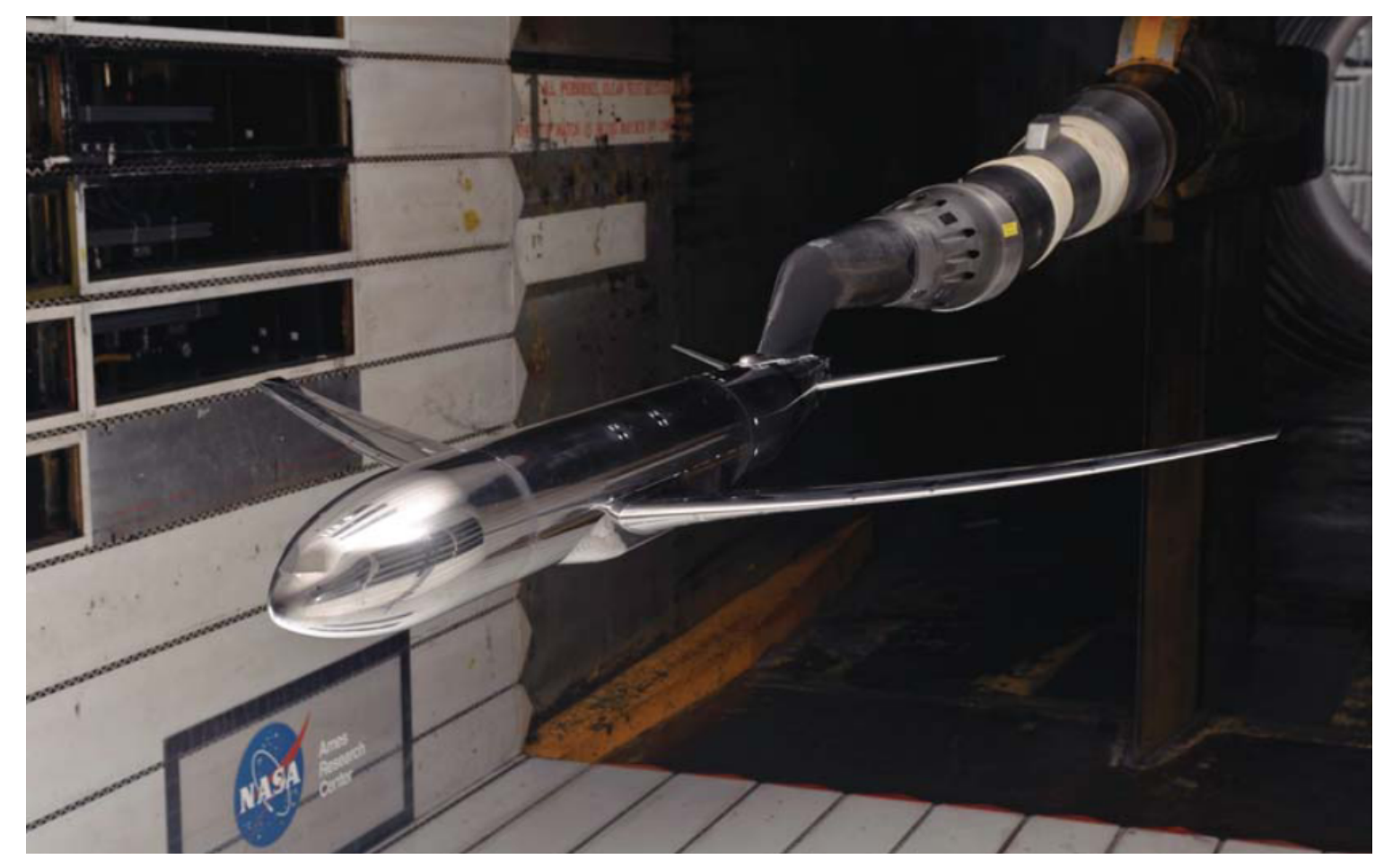

Figure 1.6: NASA Common Research Model in the Ames Research Facility ${ }^{[16]}$

non-intrusively for structural data by using videogrammetry, while aerodynamic data will be obtained using a 5-hole probe. Each of these experimental and numerical methods will be explained in greater detail in further sections. Finally, both numerical and experimental data will be analyzed, and conclusions will be made based on that analysis to determine the feasibility of FDM wind tunnel models under low aerodynic loading scenarios. 


\section{CAD MODEL}

The wind tunnel model for this research consisted of a semispan configuration of the NASA CRM with the fuselage and wing, and removing the engine nacelle as well as the horizontal and vertical stabilizers, as they were not necessary for the study. Solidworks was the software used for most of the CAD editing necessary to reshape the model as needed for the experiment. The CAD model served as a basis for providing mesh inputs for numerical simulations as well as slicing profiles for the 3D printer. Additionally, the CAD model served as a check on the deviation and manufacturing error present in the $3 \mathrm{D}$ printer from virtual model to actual model. Detailed drawings and dimensions of all parts made in Solidworks can be viewed in Appendix A

\subsection{Configuration Metrics}

Due to constraints present in the CPLSWT, edits were made on the existing CAD file of the CRM to better integrate into the test apparatus. First and foremost of these edits included scaling down the model to fit in the 3 foot by 4 foot test section. The desire for the experiment was to achieve the highest Reynolds number available in the CPLSWT without having a tunnel wall boundary layer affect the airflow over the surfaces being investigated. This led to locating the fuselage of the semispan model on the wall of the tunnel, rather than the floor, to allow a larger wingspan without penetrating a wall boundary layer. This lead to a $2.07 \%$ scale version of the full scale CRM. The dimensions of the scale model used for this study are shown in Figure 2.1. 


\subsection{Manufacturing Model}

\subsubsection{Wing Assembly}

The wings produced for this project required alterations in order to fit in the $280 \mathrm{~mm} x$ $280 \mathrm{~mm} \times 250 \mathrm{~mm}$ volume available on the Lulzbot Taz 6 3D printer. The semispan wing for the project, seen in Figure 2.1, needed to be split in a minimum of three sections to appropriately fit on the print bed. For notation purposes, these three sections are denoted "tipwing" for the outermost section, "midwing" for the middle section, and "basewing" for the innermost section. This notation was used for the duration of the project, and can be seen in Figure 2.1.

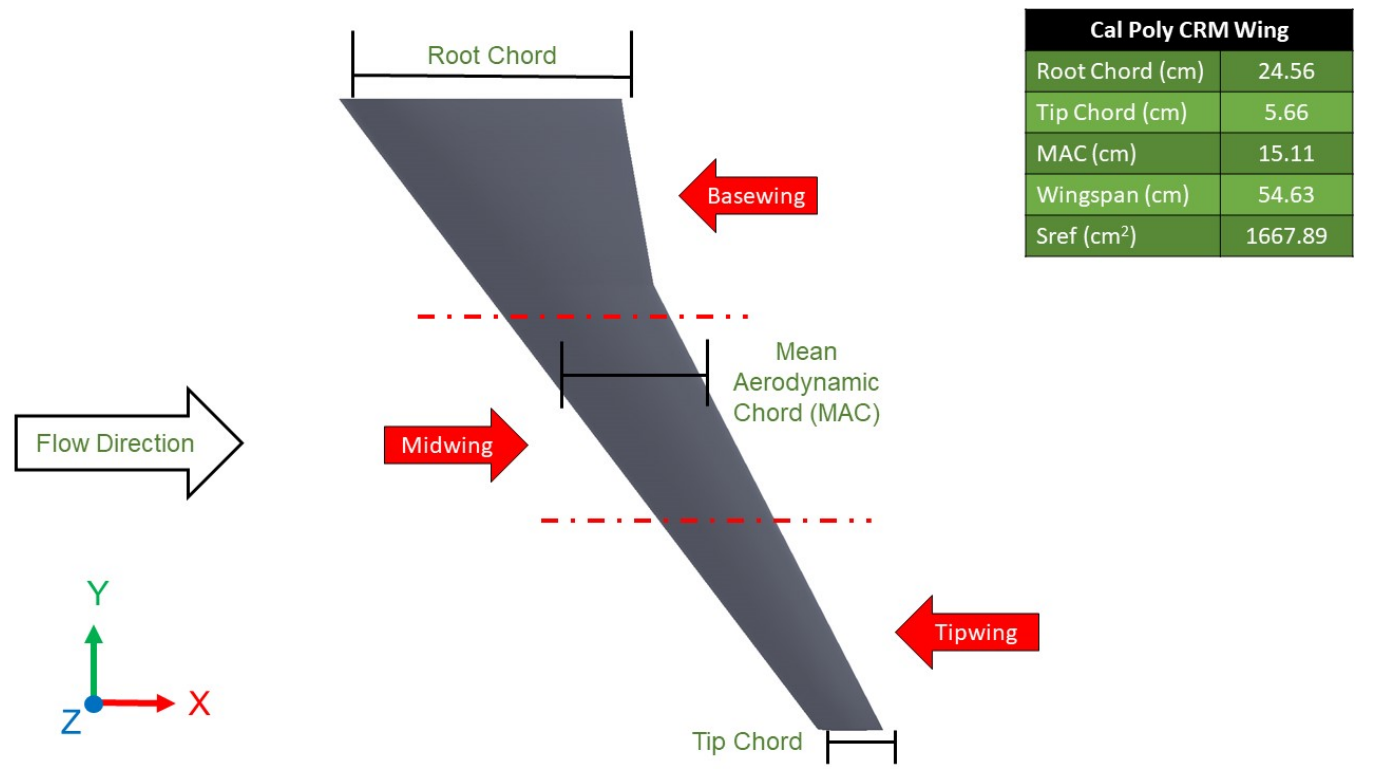

Figure 2.1: CAD model of the CRM wing used for the CPLSWT

In order to slot the wing pieces properly so that they are aligned and act as a continuous piece after post-processing, a male and female slotting configuration was introduced to each section. These extrusions and cuts into the CAD model consisted of ellipses that were extruded parallel to the leading edge of the wing such that they did not protrude past the outer mold line of the joining section. The process was produced first on the tipwing, in the 
form of a male extrusion. The male extrusion of the tipwing then produced a female cut into the midwing using a cavity command in SolidWorks. The cavity was then inflated by approximately $5 \%$ to account for the printer extrusion widths, imperfections, and tolerances. The result of these edits is shown in Figure 2.2.

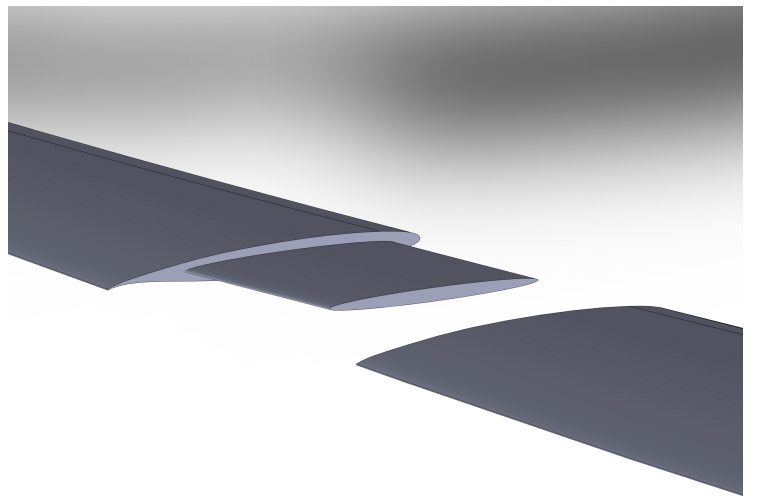

(a) Isometric view of the tipwing male extrusion

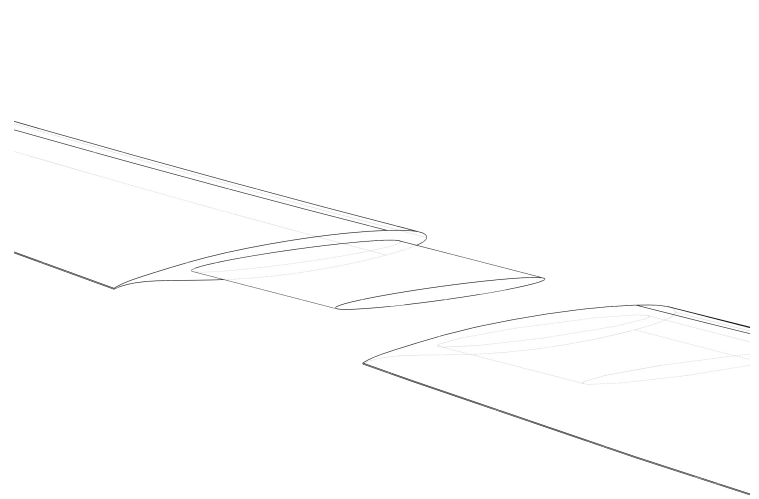

(b) Transparent view for observation of the midwing female extrusion

Figure 2.2: Depiction of extrusions made in the tipwing and midwing for slotting purposes

This process was replicated for the midwing joining to the basewing, but with minor alterations to the elliptical extrusion due to the increase in surface area available. Finally, the extrusion for the basewing to slot into the tunnel mounting assembly was extruded perpendicular to the fuselage, rather than parallel to the wing leading edge, to reduce the complexity in the milling process used for the tunnel mounting assembly. Figure 2.3 shows these extrusions and a view of the assembly in Solidworks.

Once a confirmation in SolidWorks had been done to ensure that all pieces would fit together properly and that there was no part interference, the tipwing, midwing, and basewing were all converted to .stl files for input into Simplify3D slicing software. The file output to .stl for SolidWorks was altered to produce the highest density mesh as possible for lengths and curvatures. While this increases the file size of the part, it reduces the amount of tessellation present in the model in Simplify3D, and consequently, on the final manufactured part. 


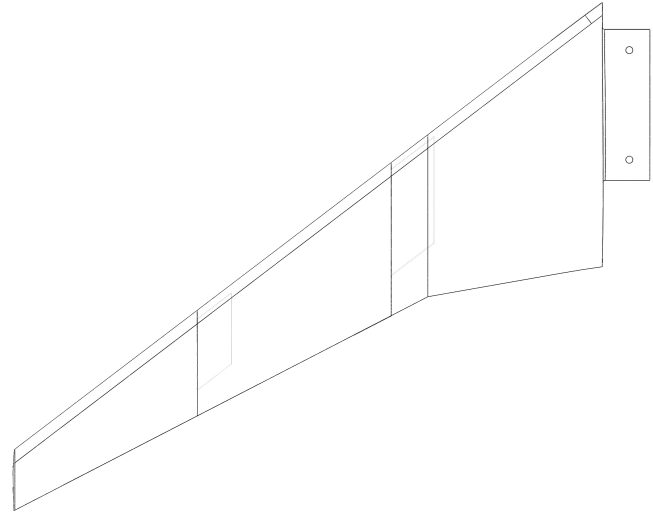

(a) Transparent view of the wing assembly

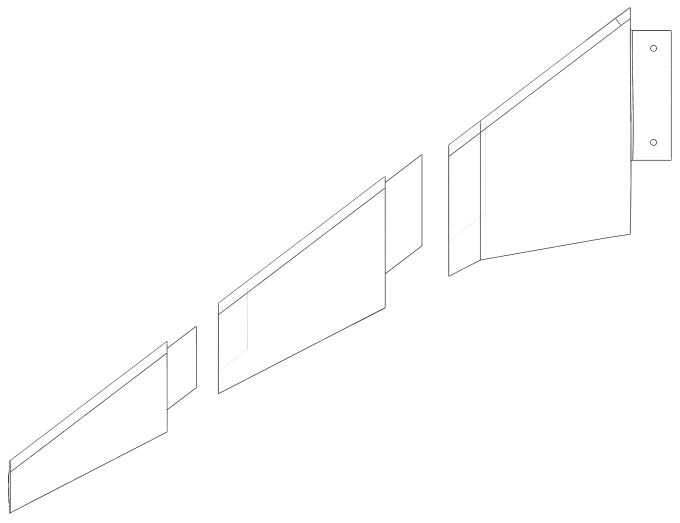

(b) Transparent view of the exploded assembly

Figure 2.3: Wing assembly construction in Solidworks prior to printing

\subsubsection{Fuselage}

The fuselage section for the manufactured part used traditional subtractive manufacturing methods, and thus was not subject to some of the constrictions present on the wing assembly. The original outer-mold-line of the fuselage is shown in Figure 2.4

Once scaled, the fuselage required further edits to allow both itself and the wing to slot to the tunnel mounting. This involved using a similar process performed in creating the slots for the wing assembly. The male extrusion created on the basewing of the wing assembly was subtracted from the fuselage. Additionally, a 5\% inflation was created during this cavity command to account for tolerancing and error. Another subtraction command was created on the symmetry plane of the fuselage to allow the tunnel mounting block to slot into the fuselage (more on the tunnel mounting block in the next section). The outcome of these edits can be seen in Figure 2.5.

After making the subtractions in the fuselage to slot both the wing and tunnel mounting block, each component needed to be held in place. Two $6.35 \mathrm{~mm}(1 / 4 ")$ holes were subtracted vertically through the wing, fuselage, and tunnel mount block to hold the assembly together. 


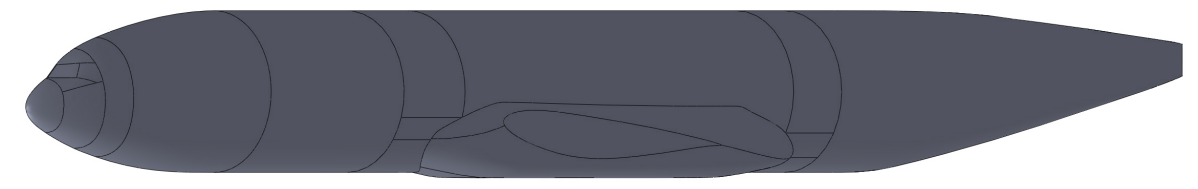

Figure 2.4: Scaled down fuselage CAD prior to OML editing
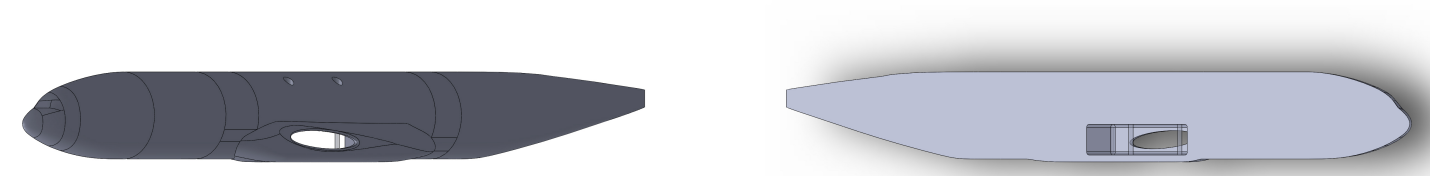

(a) Test section side of the fuselage with extruded slot (b) Window side of the fuselage with extruded slot for for the basewing the tunnel block

Figure 2.5: Cavity extrusions produced for slotting the wing and tunnel block to the fuselage

Furthermore, the holes going through the fuselage were countersunk to a diameter of $12.7 \mathrm{~mm}(1 / 2 ")$ to allow space for a nut and bolt fastening configuration. However, this 
configuration was later changed to utilize a shear pin instead of a nut and bolt combination. The result of these subtractions can be seen in Figure 2.6.

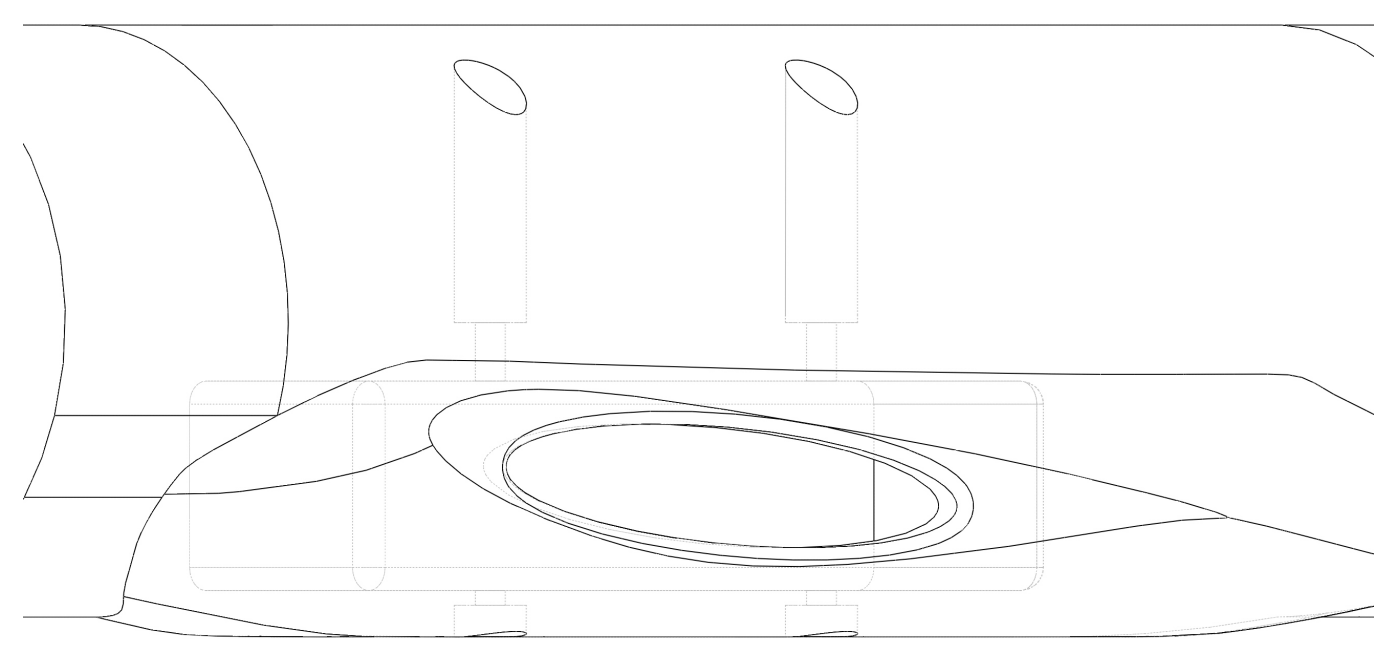

Figure 2.6: Holes cut into the assembly to allow shear pin access and removal

The last addition to the fuselage CAD model involved extruding the symmetry plane of the fuselage out in order to escape the boundary layer created by the wind tunnel walls. Assuming the flow along the wall of the test section is turbulent, which is based on the presence of a small backwards facing step occurring from the contraction to the test section, a calculation was done using Equation 2.1 for flow at $25 \mathrm{~m} / \mathrm{s}$ to estimate the height of the boundary layer.

$$
\frac{\delta}{x} \tilde{=} \frac{0.16}{R e_{x}^{(1 / 7)}}
$$


Here, the Reynolds number characteristic length is based on the length of the test section wall at $3.657 \mathrm{~m}\left(12^{\prime}\right)$, and the boundary layer location along the wall is the distance from the beginning of the test section to the nose of the fuselage, and equal to 11.10 chord lengths. Using Equation 2.1 it was found that the boundary layer thickness at the beginning of the fuselage was approximately $1.2 \mathrm{~cm}$. This number was then rounded up to an additional fuselage extrusion of $2 \mathrm{~cm}$ to ensure the fuselage was outside the wall boundary layer of the test section. Figure 2.7 depicts the additional extrusion added to the fuselage.

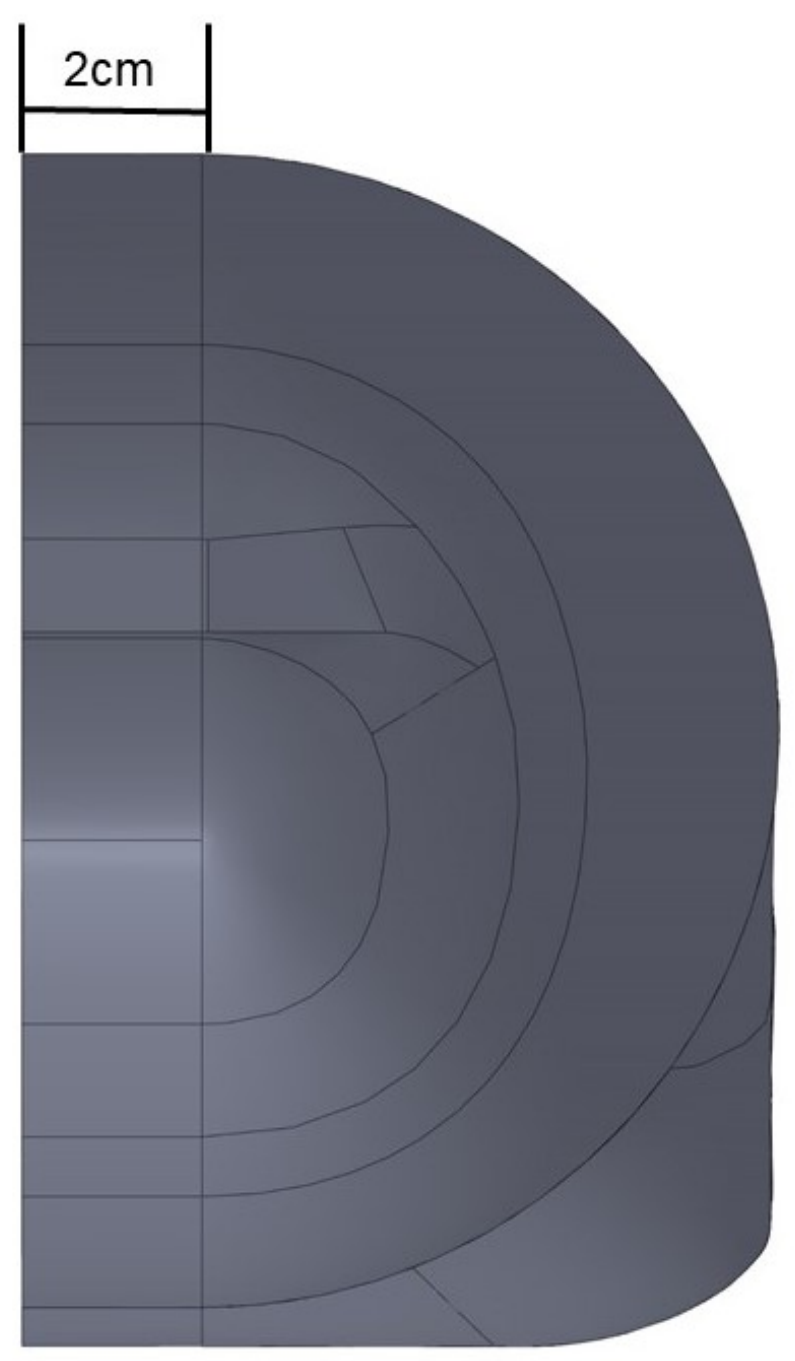

Figure 2.7: Extrusion added to fuselage to escape the tunnel wall boundary layer 


\subsubsection{Tunnel Mounting}

The tunnel mounting assembly was created in CAD from scratch to accommodate the fuselage and wing models and satisfy tunnel constraints. The tunnel mounting consists of a mounting block that attaches to the wing and fuselage, a cylinder that connects the mounting block to the outside of the wind tunnel wall, and a degree wheel with mounting holes to attach to the outside of the wind tunnel. Figure 2.8 shows these three pieces.

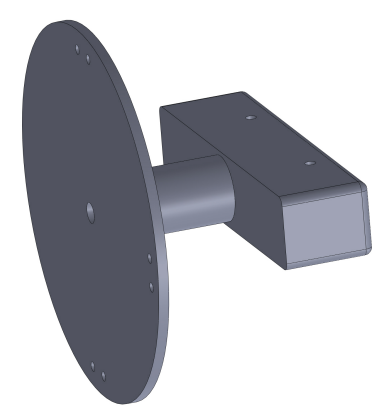

(a) Tunnel mounting hardware assembly

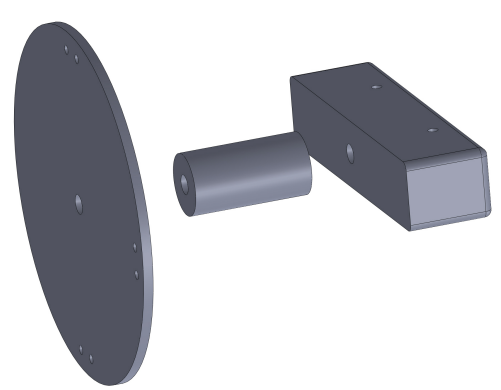

(b) Tunnel mounting exploded assembly view

Figure 2.8: CAD of the tunnel mounting hardware to hold the model stable in the wind tunnel

The cylinder is attached to the degree wheel and mounting block via two countersunk $12.7 \mathrm{~mm}(1 / 2 ")$ diameter threaded holes on either end of the cylinder. A high strength bolt combined with a locking washer is utilized to secure the assembly together. The high strength bolt ensures that there is little or no deflection occurring in the mounting

The degree wheel attaches to the outside of the tunnel wall by utilizing the T-slot extrusions present along the test section. The wheel is then bolted to the T-slot in 3 locations to ensure no translation or rotation while the test is being conducted. This design of the tunnel mounting assembly allows the cylinder section to be attached to the degree wheel, and then sent through the brush seals at the tunnel wall. The cylinder is held to the 


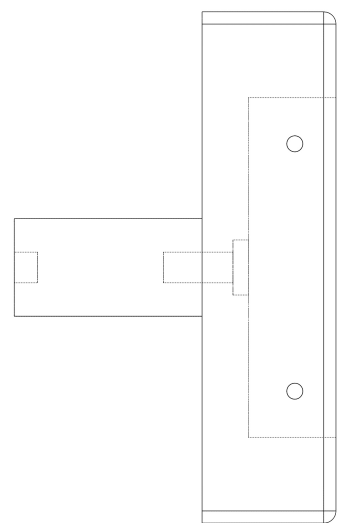

(a) Tunnel block and cylinder schematic top view

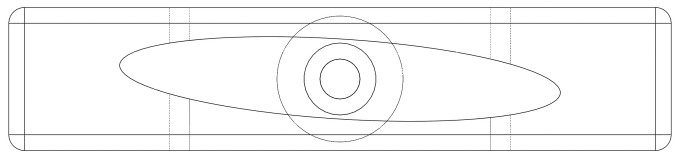

(b) Tunnel block schematic front view

Figure 2.9: See through schematic of the tunnel block and cylinder assembly

degree wheel by using an identical bolt and lock washer that was used to secure the cylinder to the tunnel block.

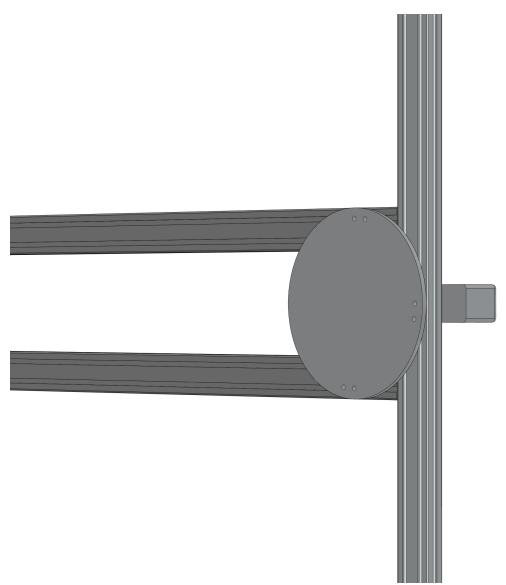

(a) View of assembly from outside the tunnel

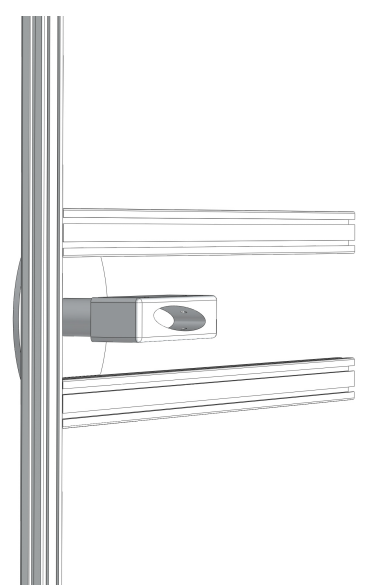

(b) View of assembly from inside the test section

Figure 2.10: Depiction of tunnel mounting assembly to test section wall

All parts of the wind tunnel model are combined into an assembly for verification. Once it has been shown that there is not part interference and that everything fits as it should, the part files are exported wither for 3D printing or CNC machining. The final Solidworks assembly for the CRM to be used in the wind tunnel is shown in Figure 2.11. 


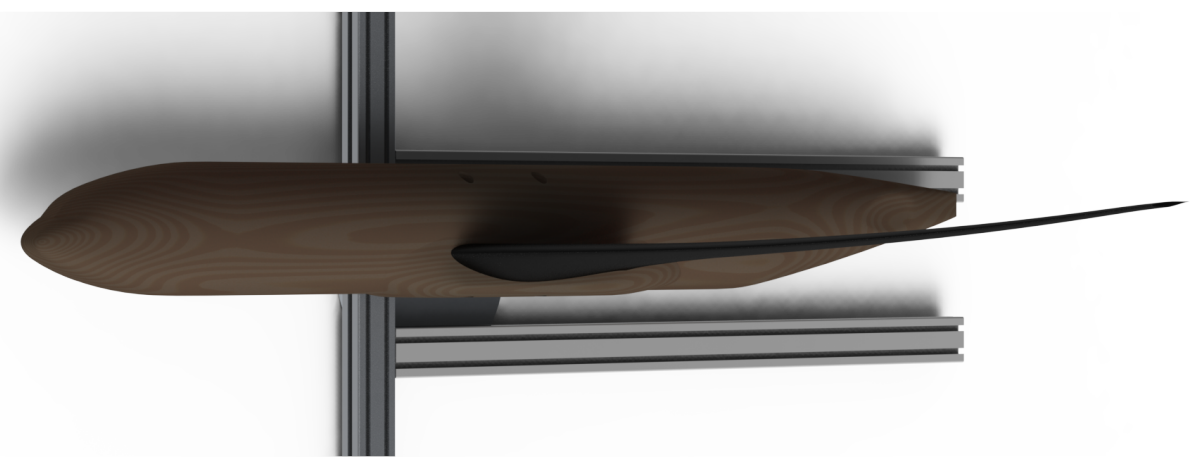

(a) 0 degrees angle of attack

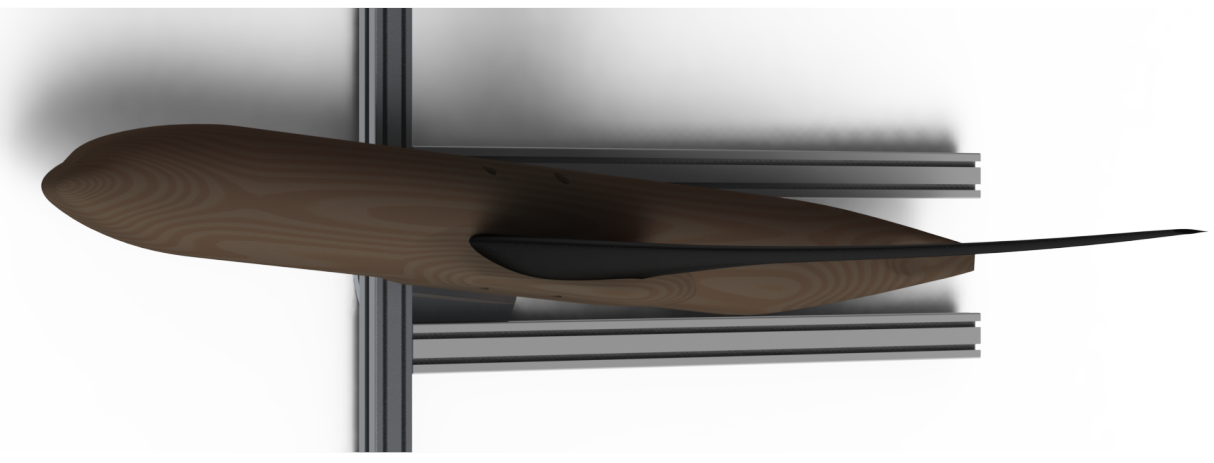

(b) 5 degrees angle of attack

Figure 2.11: Depiction of tunnel mounting assembly to test section wall

\subsection{CFD Fluid Domain}

A fluid domain for CFD purposes was created in SolidWorks utilizing the CAD model created for the manufacturing model. However, because this model is only concerned with geometries actively interacting with the flow, only the fuselage and the wing outer mold lines are used. Furthermore, the holes present in the fuselage were filled before creating the fluid domain model because these holes will be covered during the experimental process. 
The first step to creating the fluid domain was accurately measuring and modeling the dimensions of the CPLSWT test section. This resulted in a $3.657 \mathrm{~m} \times 1.168 \mathrm{~m} \times 0.863 \mathrm{~m}$ (144" x 46" x 34") test section fluid volume. Once this volume was created, the wall of the test section was aligned with the symmetry extrusion of the fuselage as it would be in the wind tunnel, with the nose of the fuselage 11.10 chord lengths aft of the beginning of the test section. After properly aligning the model with the fluid domain in SolidWorks, the fuselage and wing solid models were "subtracted" from the test section, resulting in a cavity present in the test section model seen in Figure 2.12

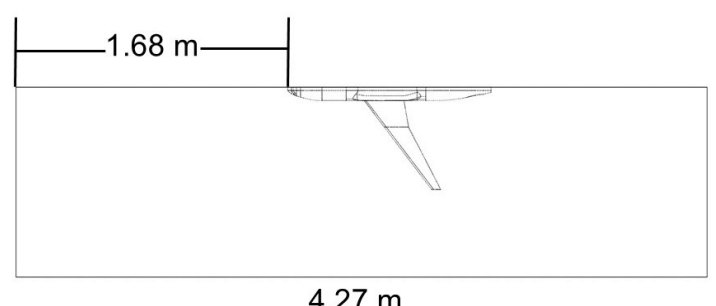

(a) Top view

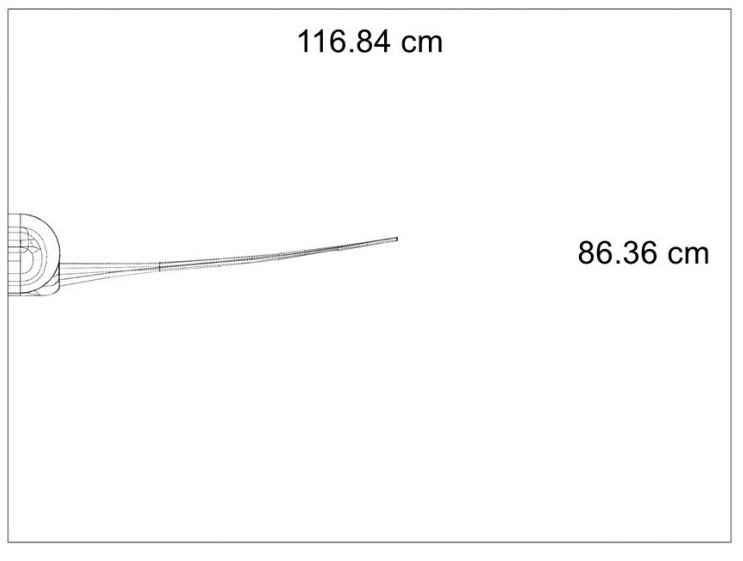

(b) Front view

Figure 2.12: Test section fluid domain used for input into CFD meshing

Flow into and out of the test section begins further out than at the beginning and end of the test section. In the wind tunnel, flow is brought in via the inlet, constricted through a contraction section, then exits the test section through a diffuser where it enters the fan. However, while it is necessary to expand the fluid volume to accommodate the flow before and after the test section, it is not necessary to directly model these tunnel components. If we assume that the boundary layer in the tunnel begins at the start of the test section, and that the flow speed is relatively constant until reaching the model, then the fore and aft sections of the wind tunnel can be modeled as volumes whose width and height are identical to the test section's cross sectional area. The length of these volumes, however, need to be long enough such that flow before the model has time to develop, and flow after 


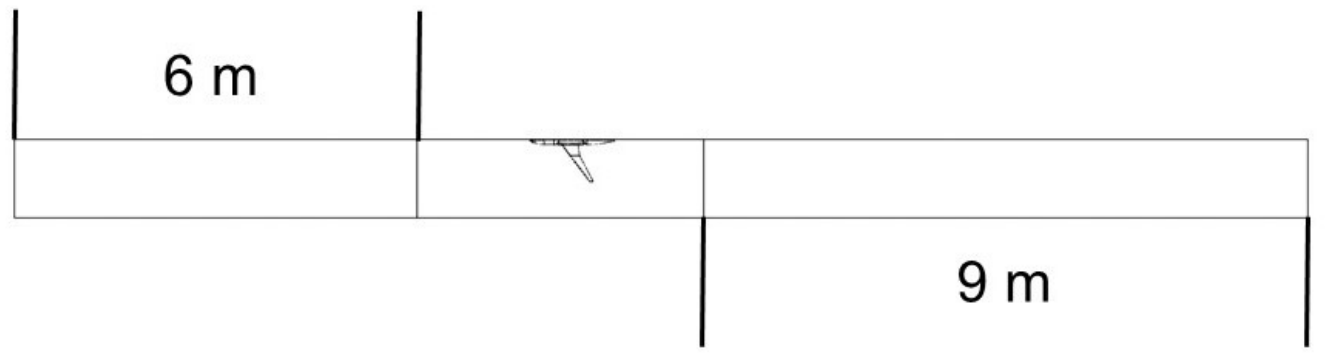

Figure 2.13: Inlet and outlet fluid domain extensions

the model is resolved before reaching the end of the domain. This understanding lead to the development of a fore section length of 39.71 chord lengths and an aft section length of 59.56 chord lengths, and can be seen in Figure 2.13

Because of the large amount of fluid movement occurring after the model in the test section, it was necessary to increase the mesh density in the area surrounding and aft of the wing and fuselage. While the meshing tool in Ansys allows the user to specify a spherical section to directly increase the density, it does not offer the user as much control from a modeling perspective. To prepare for this necessary increase of mesh density in the wake of the model, a separate body was created in SolidWorks surrounding the wing and fuselage, and extends to the end of the fluid domain. This body does not affect the fluid domain in any way, and allows the meshing tool in Ansys to increase the cell density within the volume of this body. The result is additional flow refinement in areas of interest and large flow movement. Furthermore, because the flow behind the 0 degree angle of attack simulation will be easier for the CFD software to resolve than the 5 degree angle of attack simulation, the 5 degree angle of attack wake body extends further down the fluid domain. This will cause the mesh in the wake of the 5 degree model to be finer for more of the fluid aft of the wing. The result of these body refinements can be viewed in Section 4.1.1

Finally, two more fluid domains were created in order to apply a boundary layer transition point in the fluid simulation. This is done to ensure that the boundary layer transition point for the experimental model and numerical model are relatively the same, thus reducing error 
in comparing the results. Typically, the location for this transition is placed at $5 \%$ of the local chord ${ }^{[4],[11]}$. However, due to the complexity of placing this transition point on the experimental model, the transition was placed at a constant location instead, at $7 \%$ of the MAC. This location on the fuselage was assumed to be at the beginning of the windshields of the cockpit for simplicity. Using these locations as starting points, two bodies were extruded toward the inlet of the fluid domain, one for the wing and one for the nose of the fuselage, notated "wing-body" and "nose-body" respectively. The overlapping volume between the wing-body, wing, nose-body, and nose is subtracted from the wing-body and nose-body to ensure the fluid domain in the test section is preserved. This subtraction can be seen in Figure 2.14. When these bodies are imported into Fluent, they can be specified in the software as "laminar-zones" thus ensuring the transition point is fixed in the numerical simulations.

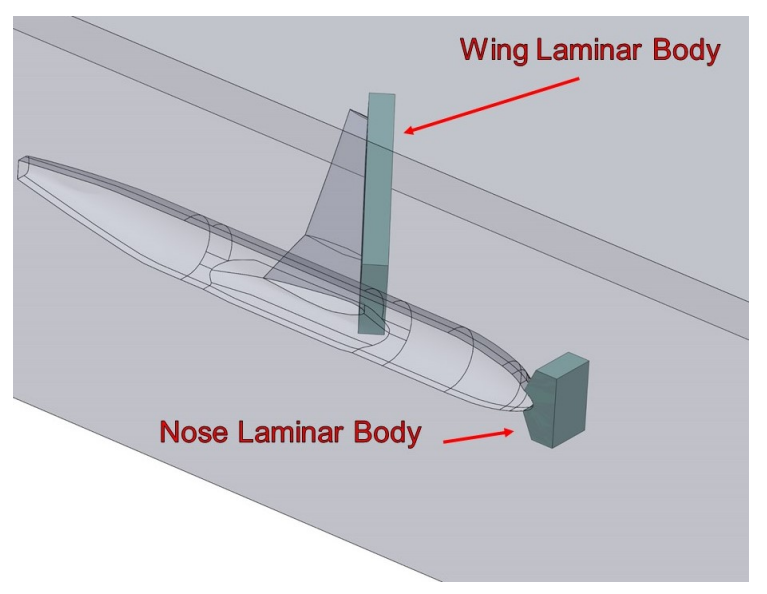

(a) Laminar bodies on the model

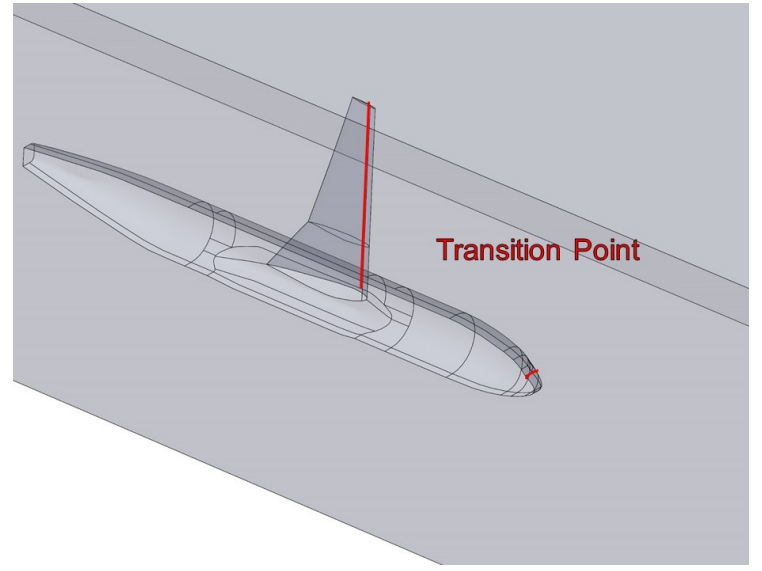

(b) Point of transition in CFD

Figure 2.14: Laminar zones for CFD laminar to turbulent transition 


\section{MANUFACTURING}

\subsection{Wing Assembly}

Three different wings were made using ABS, Nylon 618, and Nylon 910 FDM printing material to act as the experiment variables in the additive manufacturing processes. Each of these materials differs in strength and stiffness, and the specifications of these materials are presented in Table 3.1 .

Table 3.1: Rapid prototyping materials for the CRM wing sections ${ }^{[12]}{ }^{[14]}$

\begin{tabular}{|c|c|c|c|}
\hline & Taulman - Nylon 618 & IC3D - ABS & Taulman - Nylon 910 \\
\hline Elastic Modulus & $153 \mathrm{MPa}$ & $986 \mathrm{MPa}$ & $665 \mathrm{MPa}$ \\
\hline Poisson's Ratio & 0.40 & 0.35 & 0.31 \\
\hline Density & $1.134 \frac{\mathrm{g}}{\mathrm{cm}^{3}}$ & $1.05 \frac{\mathrm{g}}{\mathrm{cm}^{3}}$ & $1.11 \frac{\mathrm{g}}{\mathrm{cm}^{3}}$ \\
\hline
\end{tabular}

These materials were chosen based on their variation in elasticity, relative difficulty of manufacturing using a 3D printer, and their overall use by other institutions. Nylon 618 is the most flexible material and Nylon 910 is the stiffest material, with ABS occupying the middle ground. All three materials were stored in a moisture lock out box with silica gels when not in use in an attempt to keep moisture absorption to a minimum. However, due to the marine layer present in San Luis Obispo, other measures were necessary as moisture was still being absorbed by the material. A dehydrator was used instead to "bake" each material before and during the printing process. This involved placing the material to be used inside a dehydrator at 74 degrees Celsius (165.2 degrees Fahrenheit) for 3 hours prior to printing, and continuing the dehydration process for the duration of the printing profile. This ensured that no moisture was absorbed by the specimens during printing, thus reducing the cavities produced by evaporation during the FDM printing process. 


\subsubsection{D Printing}

Once the material being used had been dehydrated and the printer turned on, a slicing profile was created for all wing sections. To maintain a degree of continuity in the wing sections, an identical slicing profile was used for the tipwing, midwing, and basewing. All slicing profiles were created using Simplify3D slicing software, and then manufactured by a Lulzbot Taz 6, Single Extruder, 3D FDM Printer.

Due to the nature of the individual materials, it was not possible to use an identical profile when manufacturing the wing sections while using ABS as compared to Nylon 618 and Nylon 910. This is primarily due to printing temperature, and how each material reacts in the real world once being heated and then cooled to room temperature. However, most of the print settings carried over between materials, especially when they simply involved geometric specifications instead of temperature specifications. These settings are presented in Table 3.2, and apply to the tipwing, midwing, and basewing profiles. Because the amount of settings used in the slicing profile is large, only the settings of major importance are presented in Table 3.2.

Table 3.2: Common slicing parameters for Simplify3D

\begin{tabular}{|c|c|c|c|c|c|c|}
\hline $\begin{array}{c}\text { Layer } \\
\text { Height }\end{array}$ & $\begin{array}{c}\text { Perimeter } \\
\text { Lines }\end{array}$ & $\begin{array}{c}\text { Extrusion } \\
\text { Width }\end{array}$ & $\begin{array}{c}\text { Infill } \\
\%\end{array}$ & $\begin{array}{c}\text { Infill } \\
\text { Structure }\end{array}$ & $\begin{array}{c}\text { Top Solid } \\
\text { Layers }\end{array}$ & $\begin{array}{c}\text { Bottom Solid } \\
\text { Layers }\end{array}$ \\
\hline $0.2 \mathrm{~mm}$ & 2 & $0.6 \mathrm{~mm}$ & $50 \%$ & $\begin{array}{c}\text { Honey- } \\
\text { comb }\end{array}$ & 5 & 4 \\
\hline
\end{tabular}

The major differences between materials used to manufacture the wings are shown in Table 3.3, and are primarily concerned with heating and cooling the materials as they print, though there are additional settings listed that are not temperature dependent. Another difference in the printing of the materials is that the Nylon configurations were required to print without ooze control. This forced the printer to seldom, if ever, lift up from its current 
printing position and traverse to a new position. This allowed less control over where the nozzle begins and ends a layer extrusion, thus limiting the ABS to the same regulations.

Table 3.3: Varied printing parameters for Simplify3D

\begin{tabular}{|c|c|c|c|}
\hline $\begin{array}{c}\text { Print } \\
\text { Temperature } \\
\left(C^{o}\right)\end{array}$ & Nylon 618 & ABS & Nylon 910 \\
\hline $\begin{array}{c}\text { Bed } \\
\text { Temperature } \\
\left(C^{o}\right)\end{array}$ & $\begin{array}{c}110, \text { Ramped down by 5 } \\
\frac{C^{o}}{2 \text { layers }}, \text { 65 after 18 layers }\end{array}$ & 110 & 250 \\
\hline $\begin{array}{c}\text { Cooling Fan } \\
\text { Filament } \\
\text { Diameter }\end{array}$ & Off & $\begin{array}{c}\text { Linear ramping for layers } \\
\text { less than 15 seconds }\end{array}$ & Off \\
\hline $\begin{array}{c}\text { Print Bed } \\
\text { Material }\end{array}$ & PEI glass and PVA glue & $3 \mathrm{~mm}$ & $2.85 \mathrm{~mm}$ \\
\hline
\end{tabular}

These settings were tested on numerous practice parts to ensure that each manufactured part retained its shape, stuck well to the print bed, and experienced little warping or splitting. However, it is understood that while steps were taken to ensure all parts produced by the 3D printer were identical to their CAD counterpart, this rarely holds true during manufacturing. The most common problem experienced when printing was material warping, layer shifts, and layer splits. All of these issues are explained in detail in the next section.

Once the print settings and STL file had been uploaded into Simplify3D, a printing preview was performed on each part to verify print settings, toolpaths, and overall print quality prior to running. The printing preview allows the user to view each individual layer or line that the toolhead will make during the user specified process. This allowed for viewing both the interior and exterior of the wing before starting the print, and an example of this for the midwing can be seen in Figure 3.1. Other preview settings can be altered for user investigation, such as active toolhead when using a multi-extruder configuration or 


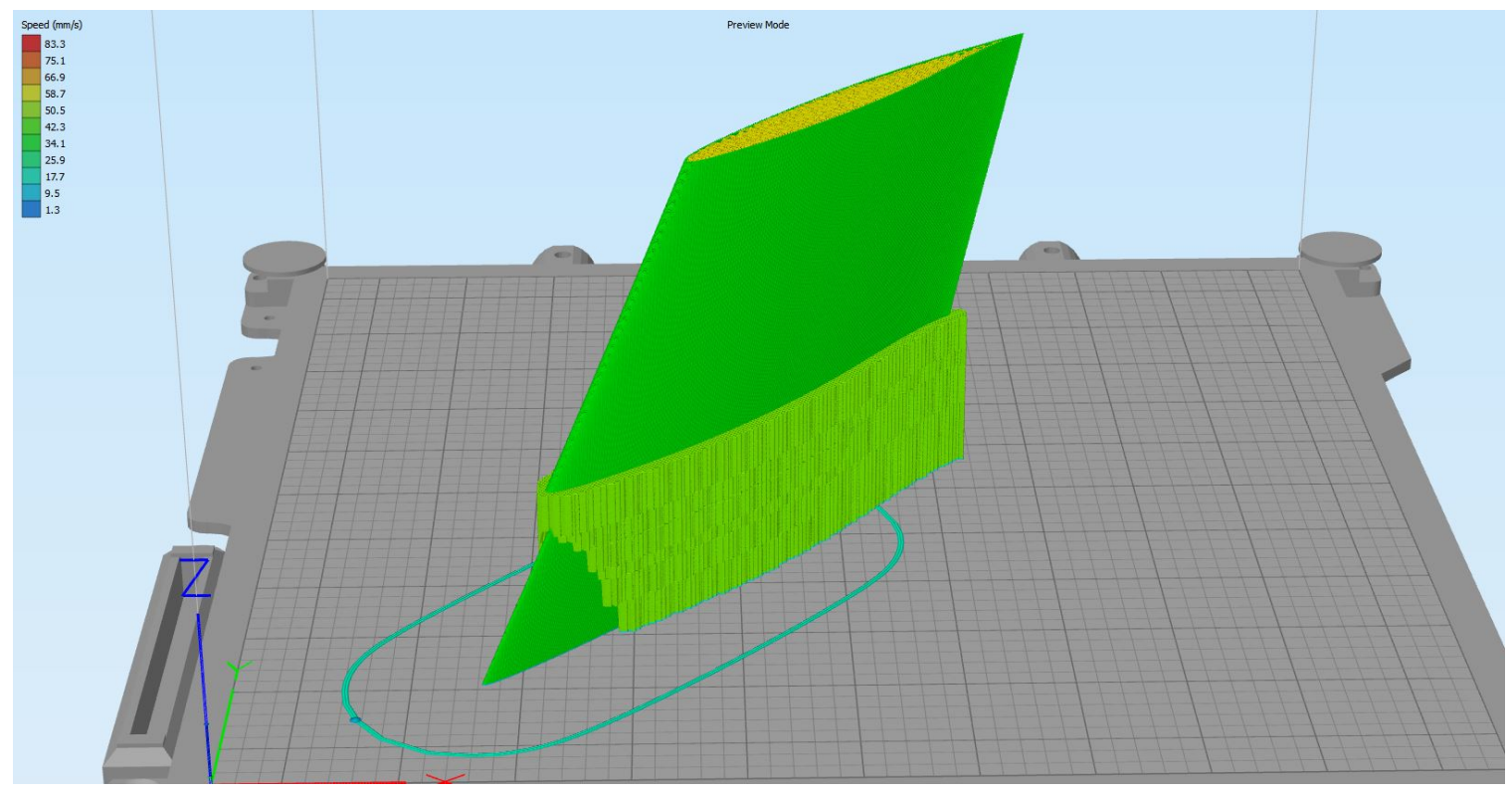

Figure 3.1: Simplify3D print preview example of the midwing at $100 \mathrm{~mm}$ above the print bed

print speed (shown in Figure 3.1). However, only print speed preview was used for wing manufacturing to verify proper cooling for ABS and Nylon parts.

After completing a verification of the print preview that the settings would behave as desired, the printing process was started. The printer would first home itself by depressing limit switches in all 3 axes, and specifying these locations as zero points. After homing, the printer retracted any filament in the nozzle by $10 \mathrm{~mm}$ to prevent oozing during the plate leveling process. Further ooze prevention was carried out through a wiping process involving the toolhead scraping across a felt strip attached to the print bed. This ensured that any filament stuck to the nozzle was removed. This is necessary because the plate leveling process required a metal-to-metal contact for electricity to pass through the nozzle. Often times, the nozzle wiping across the felt strip was inadequate to remove enough material from the nozzle to expose the metal beneath for the electric connection. When this was the case, a Scotchbrite pad was utilized to remove excess material from the nozzle. 
The bed leveling process for the lulzbot Taz 6 consisted of the nozzle on the toolhead lightly tapping each corner of the print bed twice. The corners of the print bed include a small circular metal plate, and when the nozzle touches this metal plate, an electrical connection is made and is registered by the Arduino present in the lulzbot. The Arduino then records the distance traveled in $\mathrm{x}, \mathrm{y}$, and $\mathrm{z}$ since it was homed and stores this data in a matrix. Once each corner of the plate is categorized in the matrix, geometry calculations are performed to determine if the print bed is tilted in any direction and where the hard limits of the bed are located. These geometric corrections are then applied to the slicing profile to ensure a more accurate part.

Once the plate was leveled, the toolhead began heating both the print bed and nozzle to the settings specified by the user. Once the thermoresistors present in the toolhead registered a temperature within 5 degrees Celsius of the setpoint, the extruder motor retrieved the $10 \mathrm{~mm}$ it retracted prior to leveling. Once the filament was back in the nozzle, the extrusion process began. For the CRM wings, this involved 2 skirt layers to ensure that filament was properly loaded into the nozzle, followed by the slicing profile developed in the print settings menu of Simplify3D. An example of a few wing pieces immediately after being printed are shown in Figure 3.2

\subsubsection{Post Processing and Assembly}

The nature of the manufacturing process for the wings required extensive amounts of postprocessing in order to ensure a wind tunnel ready model. This involved a large amount of sanding and filing on the surface of the part and the elliptical inserts. Because each 3D printed part is extruded in layers, and because these layers are not infinitely small, they produce "ridges" in the final part, similar to the edge of a US quarter or nickel. These ridges were sanded away from each wing piece until it was determined that the surface was smooth and no longer contained ridges. This sanding also caused the surface finish of the parts to 


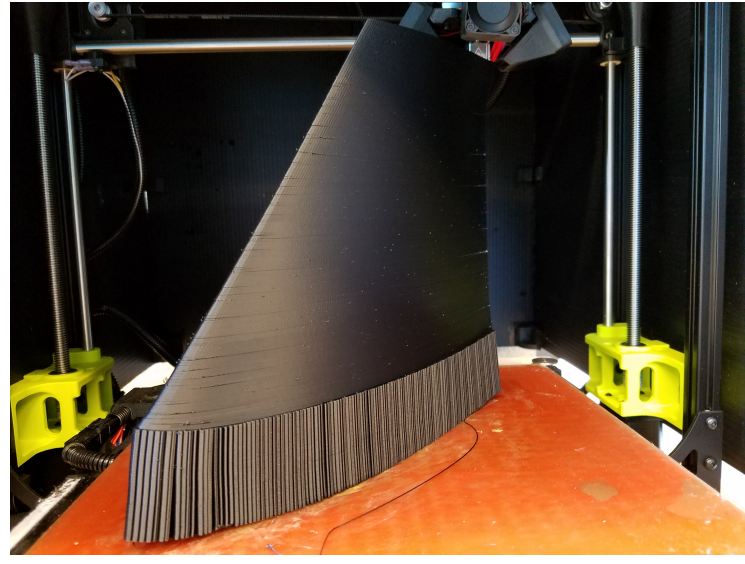

(a) ABS base wing after printing

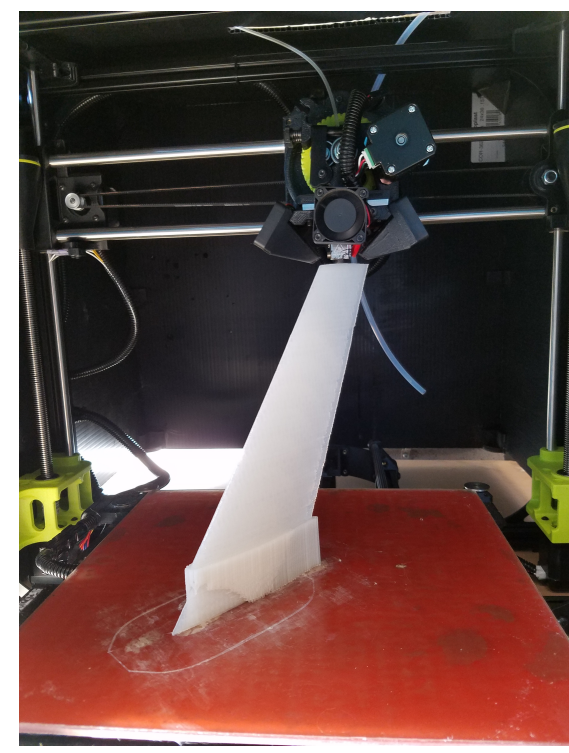

(b) Nylon 618 tip wing after printing

Figure 3.2: Example of parts after they have been printed

appear matte rather than polished, which was ideal for the wind tunnel parts. Furthermore, the tight tolerances present in the elliptical extrusions required that additional material removal be done on them so that they slotted well into its adjacent wing piece. Figure 3.3 depicts the ABS wing pieces after they had been sanded and prior to final assembly.

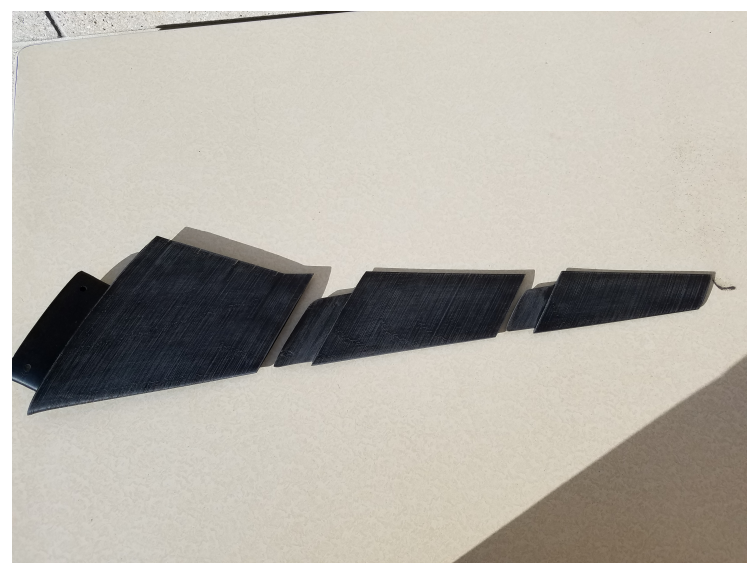

(a) ABS wing pieces after sanding

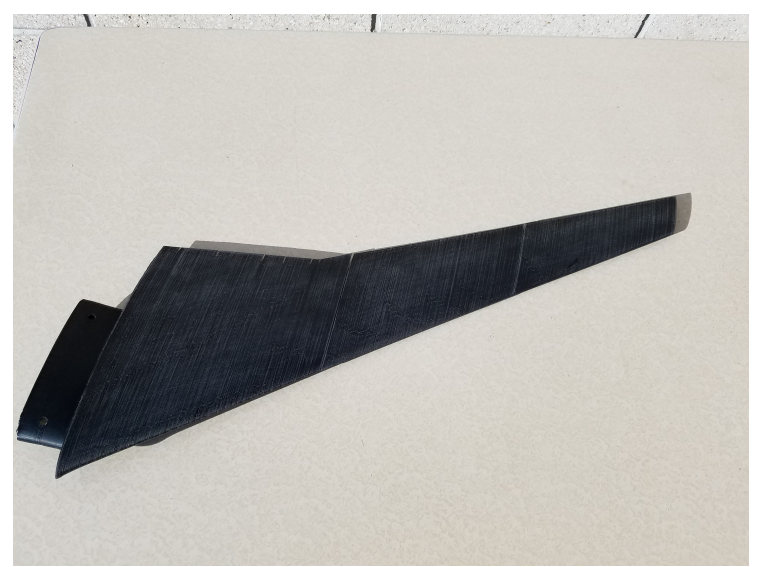

(b) ABS assembled wing pieces before epoxy bonding

Figure 3.3: Wing pieces after sanding and before bonding to showcase slotting method

Once confirmation was made that the wing pieces slotted together well with no backlash, preparations were made to epoxy the pieces together. The epoxy compound used for all 


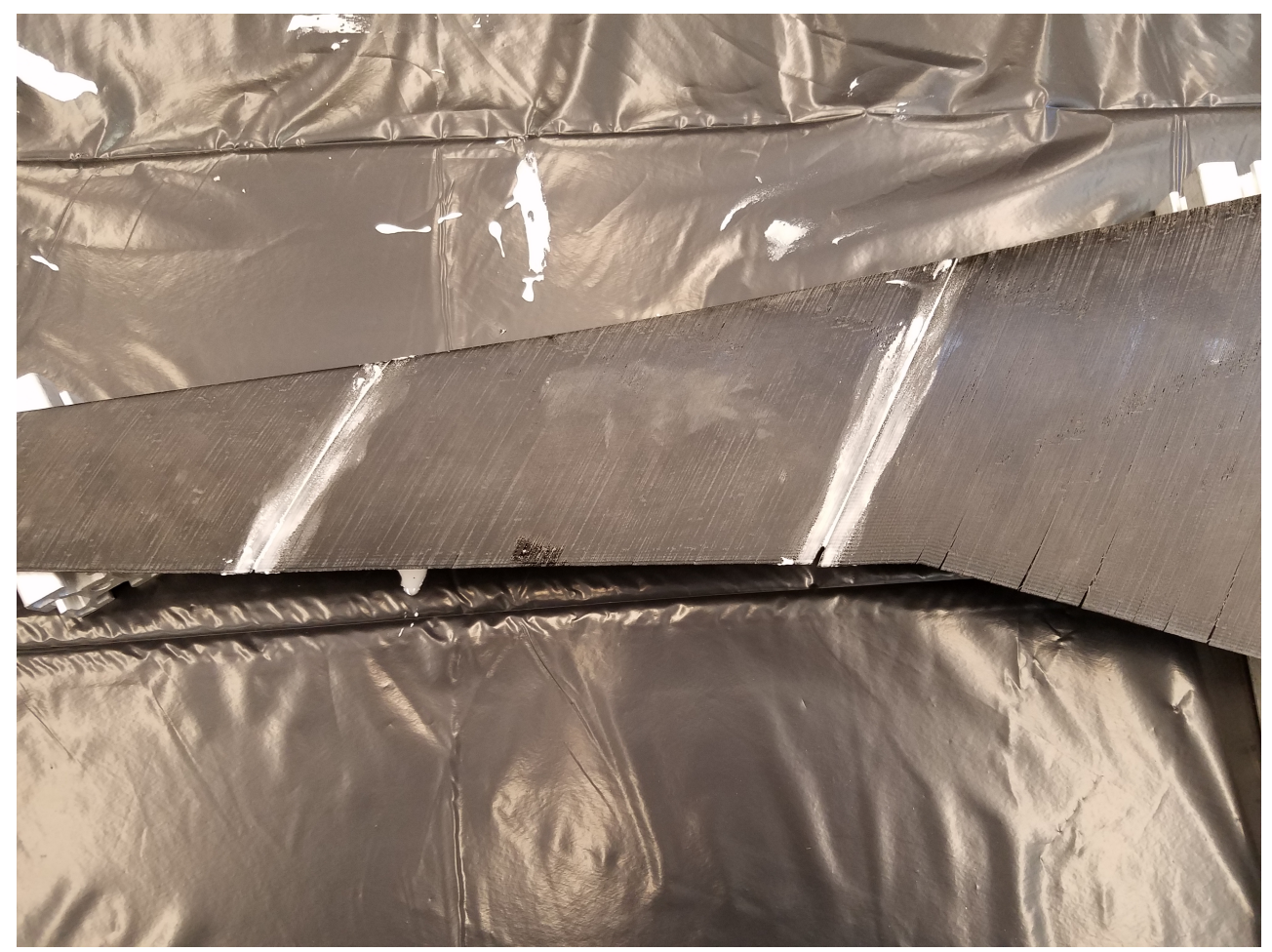

Figure 3.4: Epoxy application on the ABS wing

wing materials consisted of a West Systems Epoxy and Hardener mixture combined with glass microballoons for density variation. The hardener and epoxy were mixed according to the instructions provided with the compounds. However, the microballoons involved a much less robust process, and consisted of adding additional balloons to the mixture until a "honey" texture was achieved. Once the desired texture was obtained, the mixture was coated to the elliptical extrusion of the tipwing and midwing, and slotted into the midwing and basewing respectively. Furthermore, the mixture was coated over the seam between joining wing pieces to ensure the part was water tight once the epoxy had cured overnight. Figure 3.4 shows the ABS wing immediately after having been assembled with the epoxy mixture applied.

A 24 hour period was required for the compound to completely cure. Upon curing, additional attention to the wing was needed to account for the cracks, splits and small cavities present in the wing. Layer splitting occurs when the previous layer has cooled to 
a point where it cannot properly bond to the next layer. The layer that has been extruded, by contrast, is very hot, and begins to warp as it cools when it cannot bond properly. The result is "part splitting", is prevalent on ABS, especially in areas with thin cross sections, such as the wing trailing edge. This splitting effect can be seen in Figure 3.2.

However, due to the chemical makeup of ABS, this problem was eliminated through the use of an ABS-Acetone compound, called an "ABS slurry". Because ABS dissolves when introduced to acetone, whether liquid or solid, a chemical mixture of the slurry was made by mixing acetone with excess ABS parts. Similar to the epoxy mixture, ABS pieces were continually dissolved until the slurry reached the texture desired, with a viscosity similar to honey. This mixture was then applied to the splits, cavities, and other defects in the wing pieces to create a water tight, continuous wing that is still composed of only the ABS plastic and epoxy compound. It should be noted that this process was not necessary for the nylon materials due to their robust bonding properties with subsequent layers from the extruder.

After filling gaps in the wing materials after the assembly, the wings were painted with matte black paint. The primary purpose for this painting was to reduce laser reflection during PIV testing. Because the matte black paint absorbs more light than other colors, less laser light can reflect off the surface, reducing potential harm to both people and cameras. The paint also acted as a filler compound to even out the ridges left behind by the printing process that sanding could not remove. In light of this fact, each wing underwent 3 coats of painting and sanding before a final spray paint application. Figure 3.5 shows the an example of this paint after being applied to the ABS wing, and Figure 3.7 shows both the wing and fuselage after being completely post-processed and ready for wind tunnel testing. 


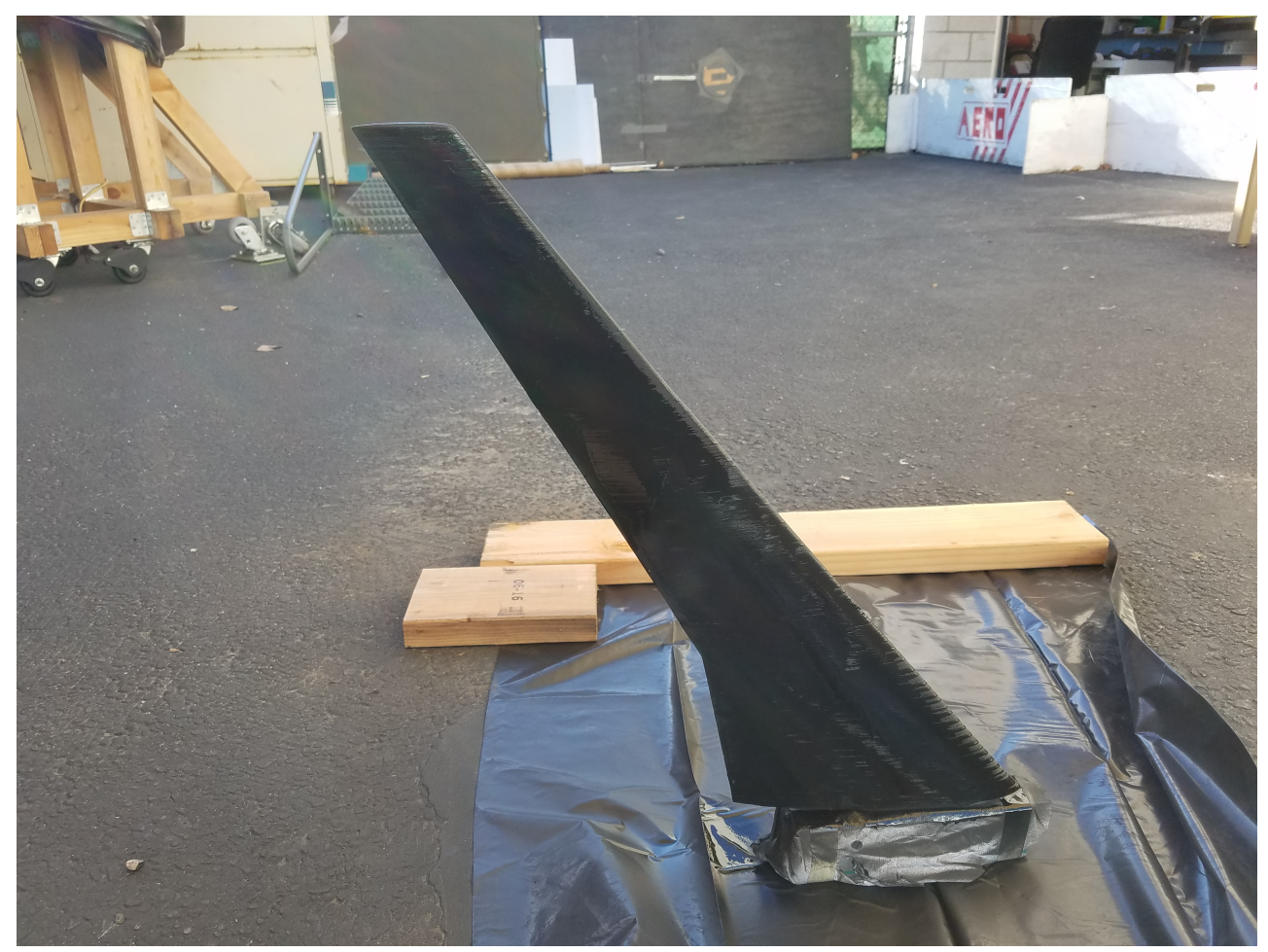

Figure 3.5: First coat of paint on the ABS wing

\subsection{Fuselage}

The fuselage manufacturing involved using a $\mathrm{CNC}$ machine to create the contours provided by the Solidworks model. Originally, the fuselage was to be made of foam for simplicity factors. However, upon observing the small cross sectional area present at the intersection between the tunnel mounting and the fuselage, the material was changed to wood to avoid any fracturing of this area. Furthermore, because of the shape of the fuselage, it was necessary to manufacture the body in two sections. The body was split after the cavity for the tunnel mounting and wing, and can be seen in Figure 3.6. Figure 3.6 shows the fuselage sections after a coat of primer has been applied to them as well as after they have been sanded. Similar to the wings, sanding could only take out so many ridges left behind from the manufacturing process. This forced the fuselage to undergo the same amount of painting as the wings to ensure an even and smooth surface for wind tunnel testing. 


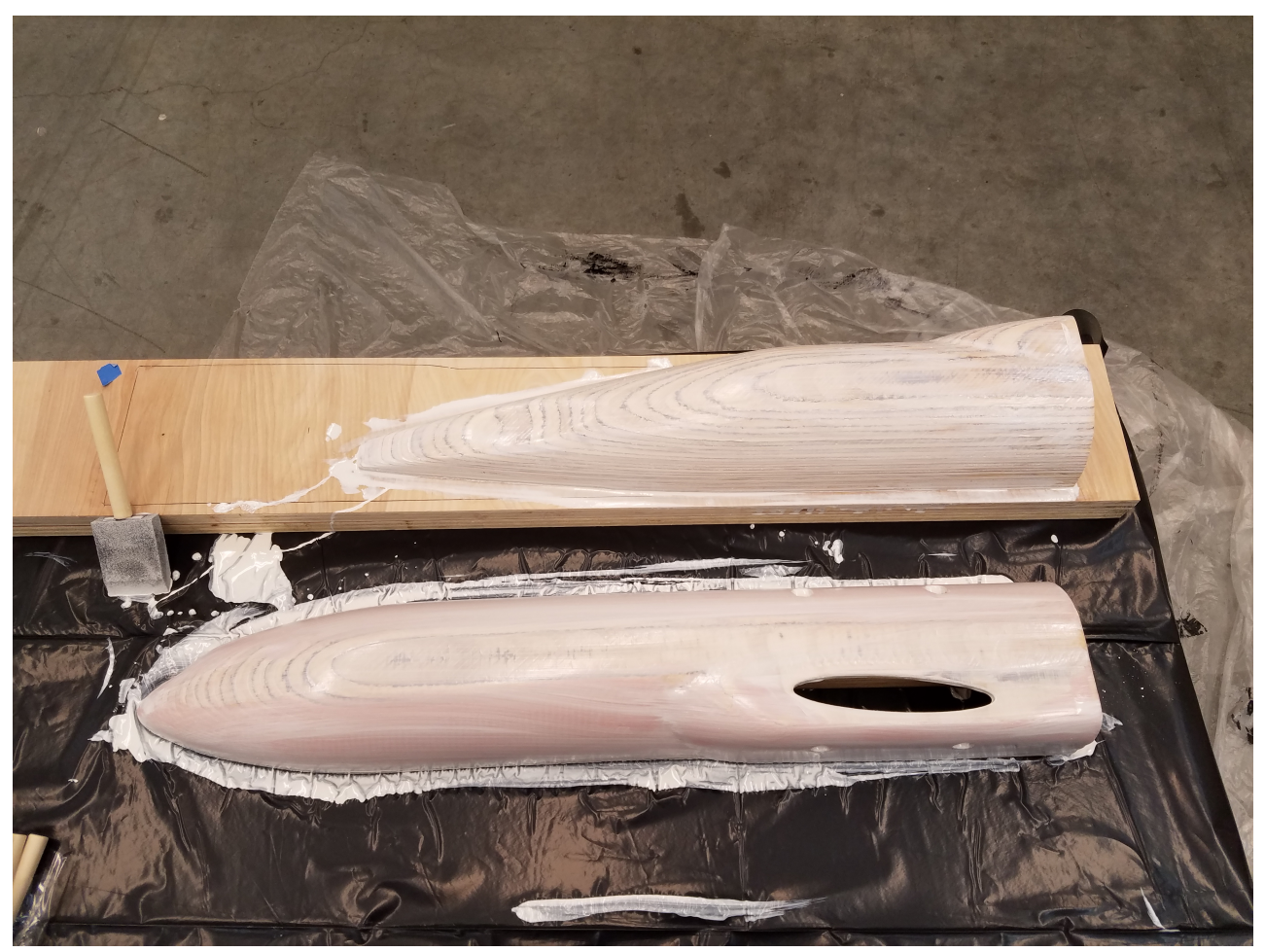

Figure 3.6: Fuselage sections after having primer applied to the wetted side

In order to bring the parts together to act as one unit, the same epoxy compound used on the wings was used to join the two fuselage sections together. Because the fuselage is heavier than the wings, it was decided that more was needed to ensure that the two pieces stayed together. This was accomplished by bolting the additional $2 \mathrm{~cm}$ boundary layer extrusion to the fore and aft sections of the fuselage. These bolts were applied on the window side of the fuselage such that the attach well to each section without intruding into the wetted area of the fuselage. After 3 coats of paint and additional sanding, the wind tunnel model was ready for testing, and can be seen in Figure 3.7

\subsection{Wall Mounting}

The mounting assembly for the model was made entirely of aluminum as it was a load bearing member of the experiment. The mounting consisted of wind tunnel block, cylinder connector, and degree wheel mount. The three parts can be seen in Figure 3.8. The degree 


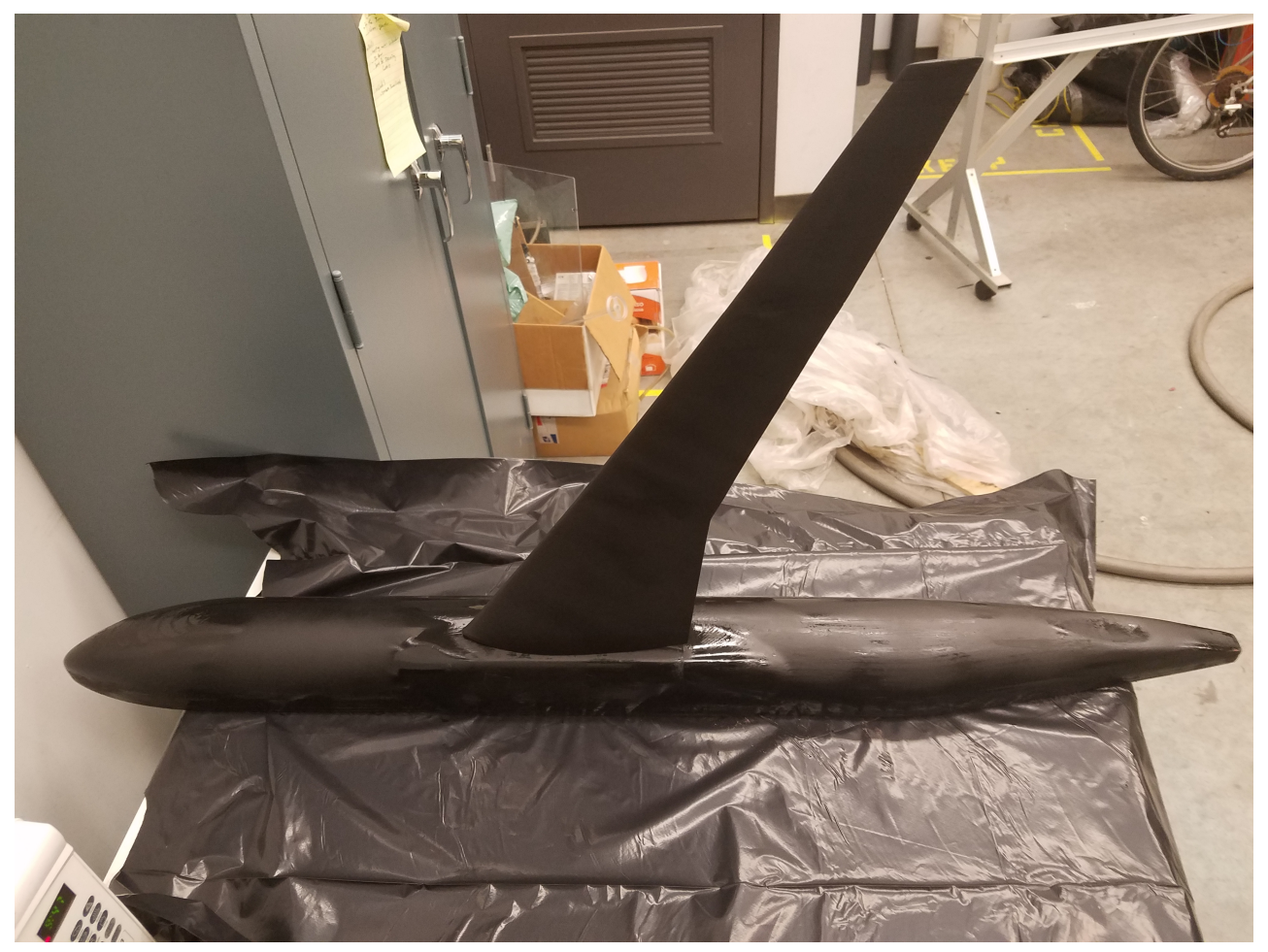

Figure 3.7: Final test ready model with the ABS wing

wheel and tunnel block were connected to the cylinder using two high carbide 0.5 " bolts and lock washers to increase bolt tension and reduce slippage.

The degree wheel was mounted to the outside of the wind tunnel wall, as depicted in Figure 3.9a. The mounting configuration was bolted down in three places to ensure no rotation or translation along the T-slot extrusions along the tunnel wall. Furthermore, the cylinder diameter was made large enough to reduce deflections due to aerodynamic loading induced by the wing of the model, but small enough such that it fit through the brush seals present in the tunnel wall. The assembly before applying the tunnel block can be seen in Figure 3.9b

The tunnel block was then attached to the end of the cylinder after the degree wheel had successfully been mounted to the outside of the tunnel. The block could not be attached beforehand because it was too wide to fit through the brush seal slot in the tunnel wall. The block was fastened to the cylinder using one of the high carbide bolts and a lock washer. 


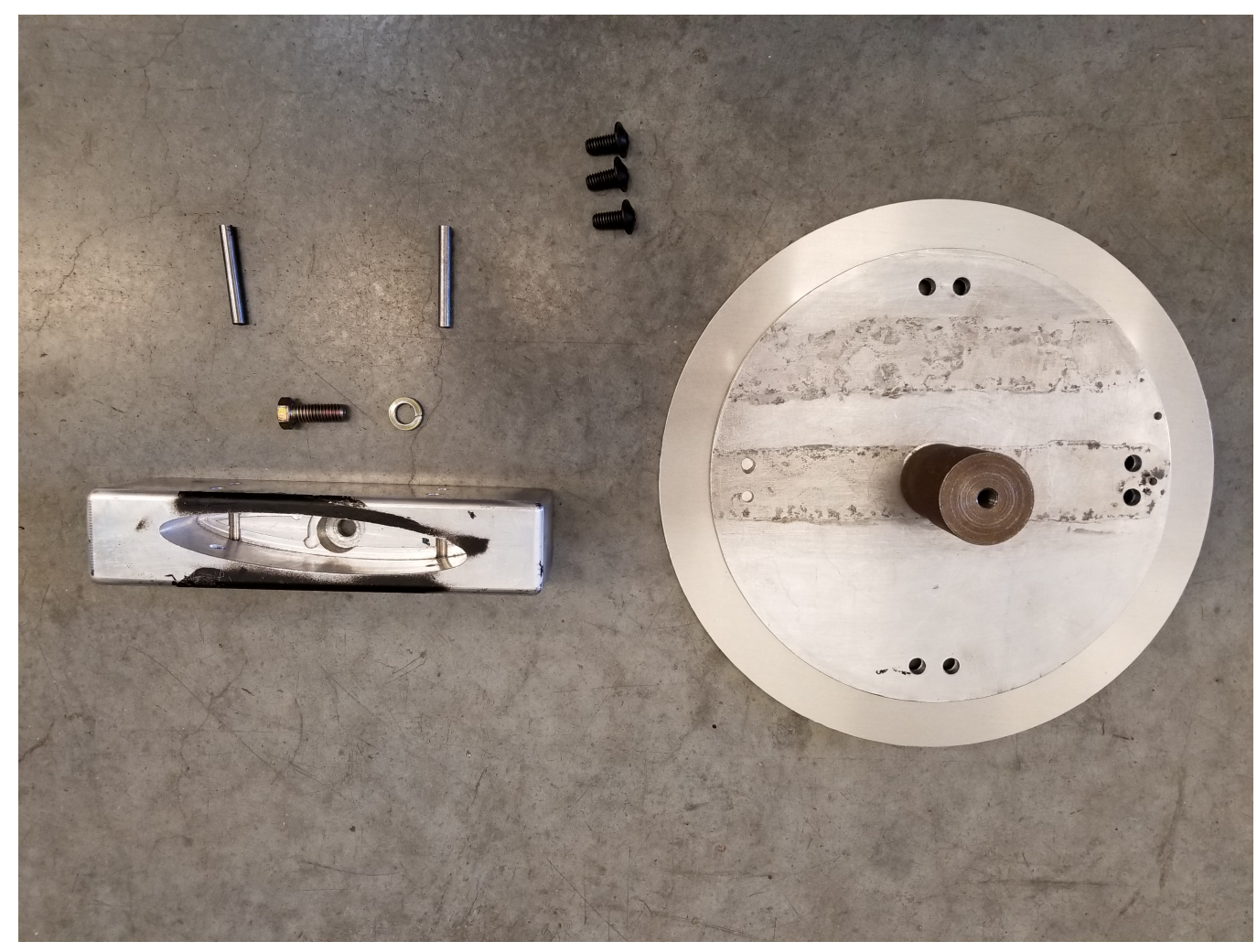

Figure 3.8: Individual parts used in the tunnel mounting assembly

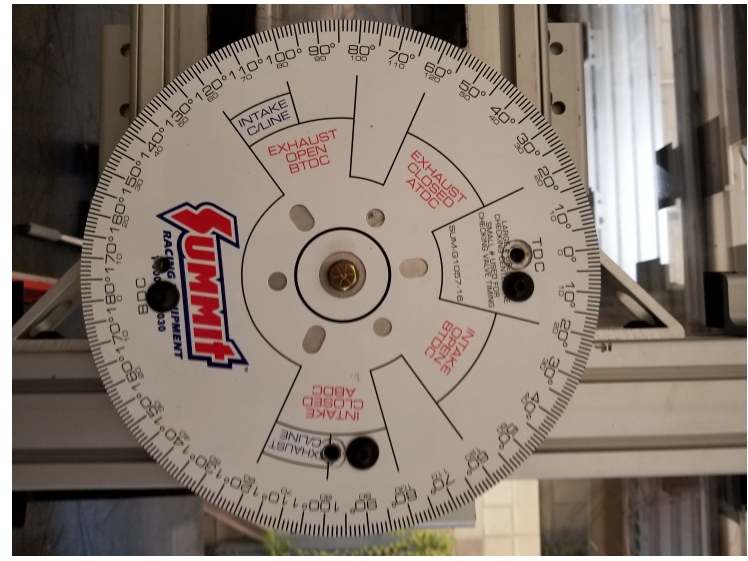

(a) Degree wheel mounting to the outside of the tunnel

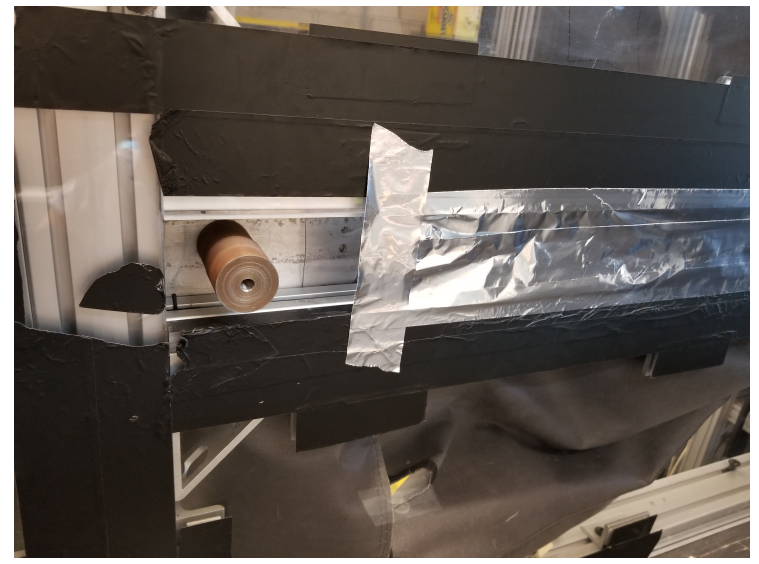

(b) Cylinder protruding through slot in the tunnel wall from the degree wheel

Figure 3.9: Mounting assembly prior to the attachment of the tunnel block

To ensure that the degree wheel rotation matched the block, the degree wheel was set to 0 degrees and a digital level was placed on the block during assembly to ensure that the angle of the block was also 0 degrees after tightening the bolt. The mounting assembly after attaching the block can be seen in Figure 3.10. 


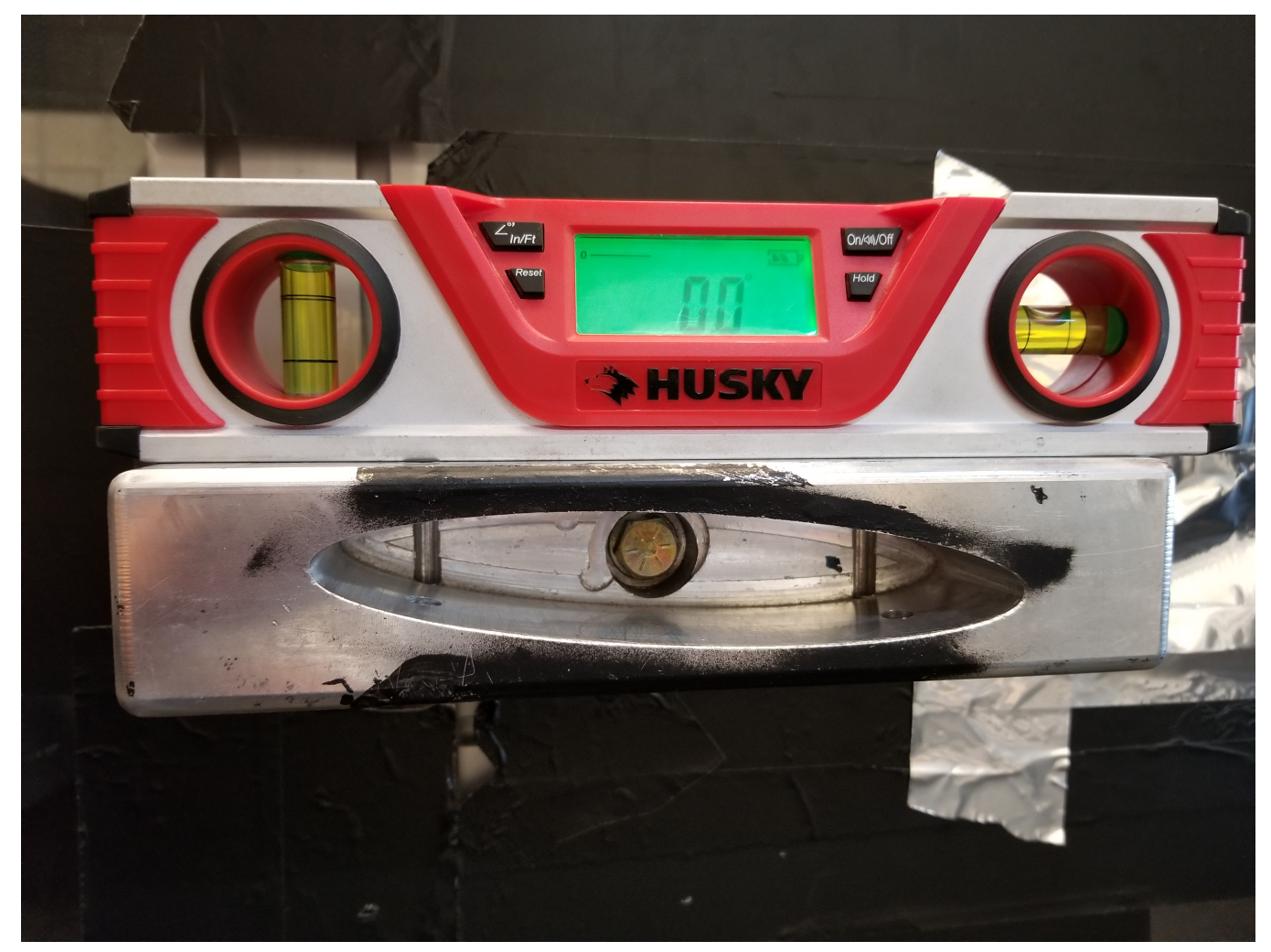

Figure 3.10: Tunnel block attachment with a digital level for precise angle of attack

After the tunnel mount assembly was complete, the fuselage was slotted onto the wind tunnel block until the back of the fuselage was flush with the test section window. Once slotted, the wing could be inserted through the fuselage and into its place in the mounting block. Finally, the fuselage and wing were kept in place on the block using two 0.25 " shear pins through the holes in the fuselage, wing, and mounting block. These holes present in the fuselage were then covered with matte black aluminum tape to create a more water-tight model. The model was then ready to begin testing, and can be seen in Figure 3.11 


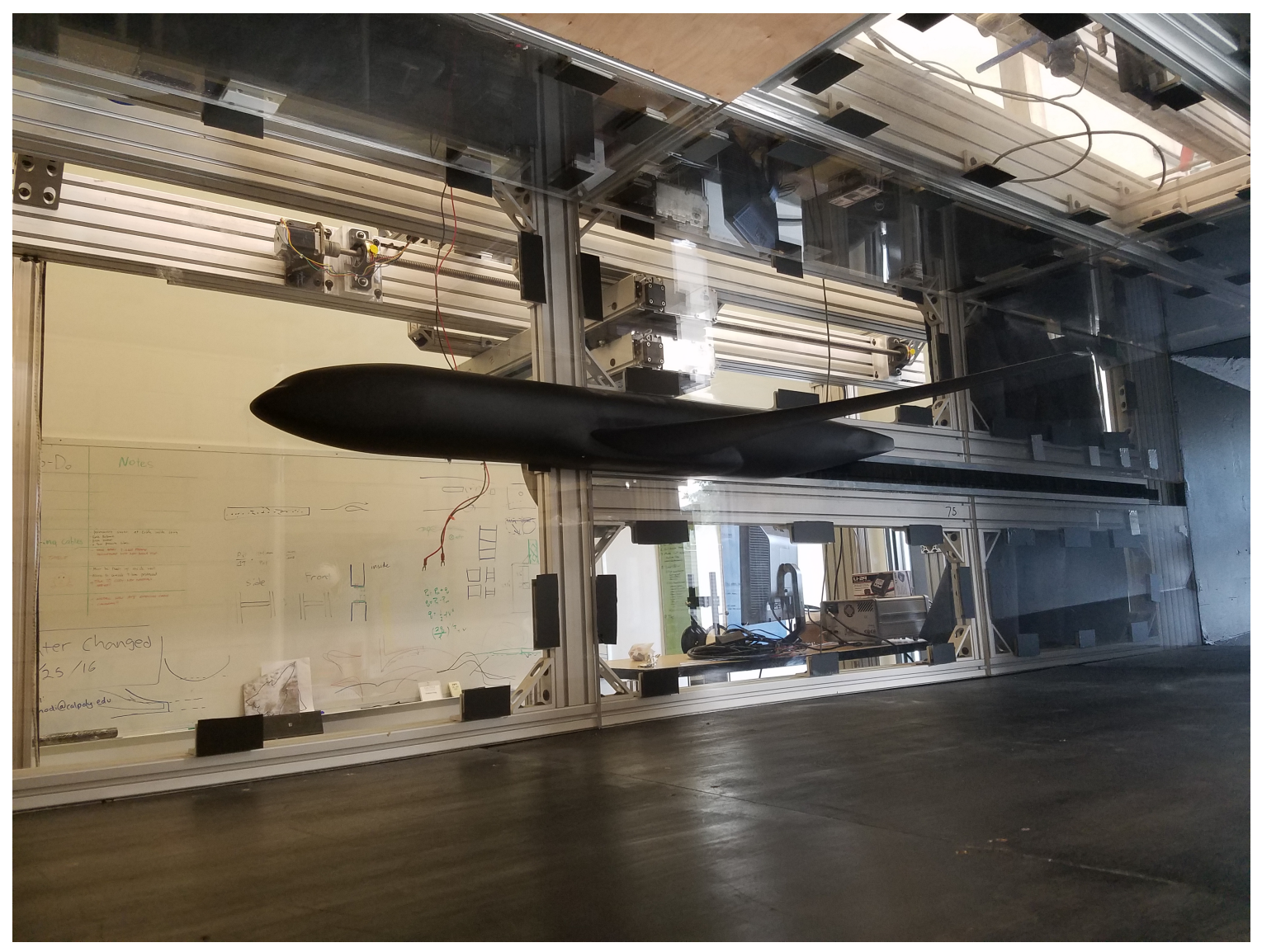

Figure 3.11: Wind tunnel model installed in the test section 


\section{NUMERICAL SIMULATION}

\subsection{Computational Fluid Dynamics}

The CFD portion of the numerical simulations involved importing the fluid model from Solidworks to be meshed by Ansys Mechanical, solved by Ansys Fluent using a steady state RANS simulation, and post processed in FieldView. The process also involved saving and exporting pressure loads along the wing surfaces such that they could be imported as mechanical loads for the FEA simulations.

\subsubsection{Meshing}

Mesh settings for the fluid domain were selected such that it met the requirements for the turbulence models selected for processing with enough quality to satisfy a convergent solution. To facilitate additional quality, an advancing front algorithm was used, as the model involved primarily unstructured tetrahedron cells. The regions denoted for the nozzle and contraction bodies utilized a hexehedral mesh due to their simplistic geometry. The primary global settings for the CFD model at 0 and 5 degrees angle of attack are displayed in Appendix B. These are not all the settings available for the global mesh changes in Ansys, but simply the values that have been altered from their default setting to produce a finer, higher quality mesh.

These settings help refine areas in the fluid domain, however additional refinement was necessary for areas closer to solid bodies and the wakes of those bodies. The first additional refinement was to increase the mesh density in the area before and after the wing by utilizing a body of influence command. This told the mesher to create higher density cells where 
the fluid body intersected any portion of the "mesh body" created in Solidworks, and can be seen in Figure 4.1 for 0 degrees angle of attack. This is the only case where the 0 and 5 degree angle of attacks mesh settings differ. Because the flow in the wake of the 0 degree simulation was found to be relatively simple and easy to resolve, a smaller body was used. Both cases specified an element size inside the body of influence of 3.8e-003 m.

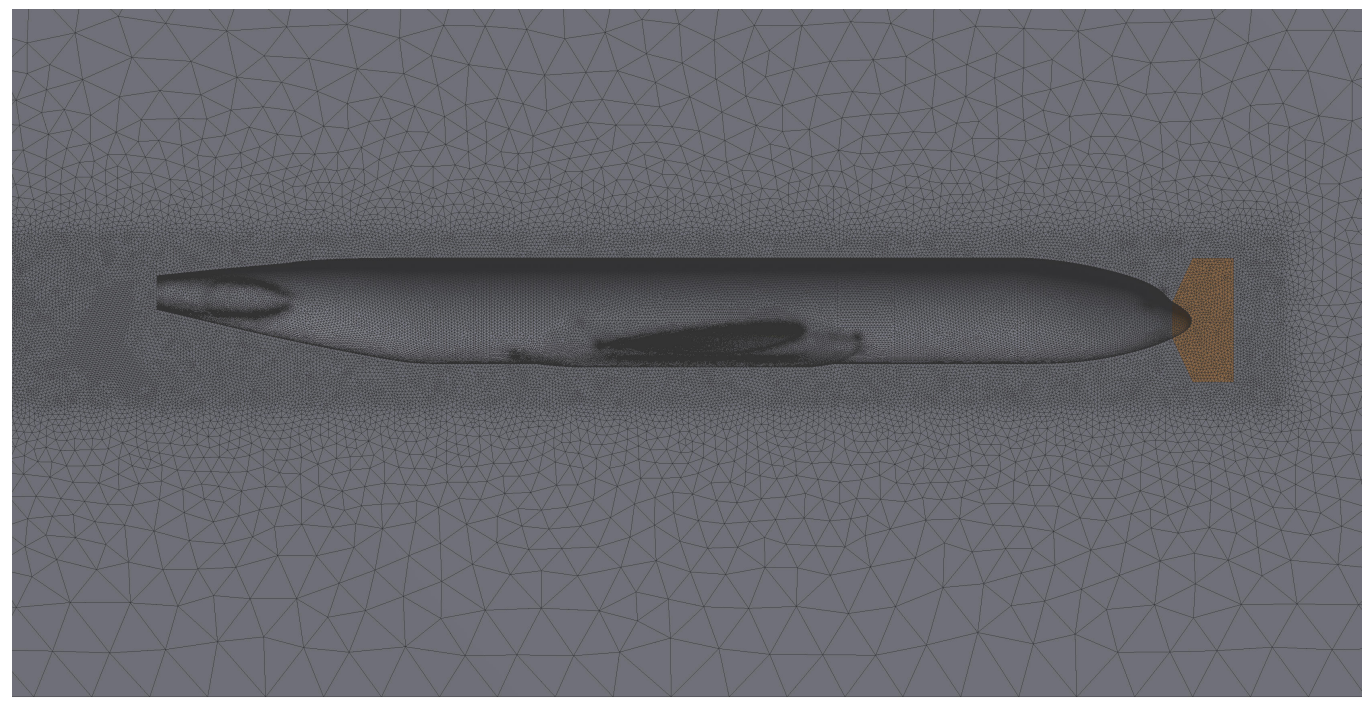

Figure 4.1: Bodies used to specify area of increased mesh density in Ansys

The next necessary change to the mesh was the addition of an inflation layer along all walls or bodies in the flow to adequately capture the boundary layer present in those areas. The wing, fuselage, and test section walls all utilized identical methods of inflation across their respective surfaces. A "last aspect ratio" option was used, with a first layer height of 4e-006 m to insure an initial $y^{+}$value of 1 , which was necessary for the turbulence models used (discussed in the next section). Furthermore, 15 layers were extended out from the surfaces with a final aspect ratio of 3 . An example of this inflation layer can be seen in Figure 4.2. The inflation layers in the contraction and nozzle bodies were produced with less fidelity to reduce mesh size and computation time. The default settings for inflation were used with the exception of extending the layers to 15 such that they matched well with the inflation layers produced by the test section walls. 


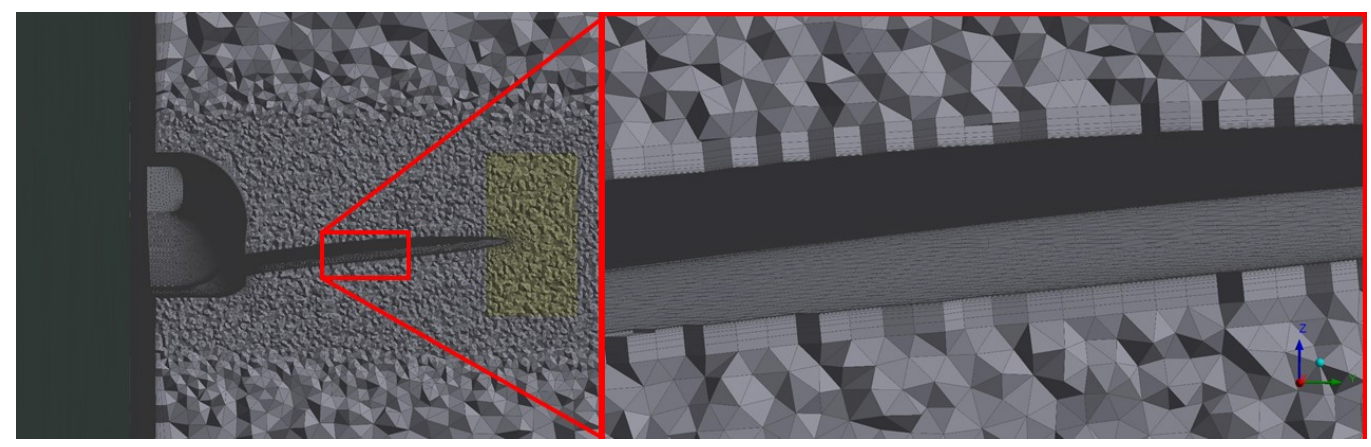

(a) X-axis slice of the mesh

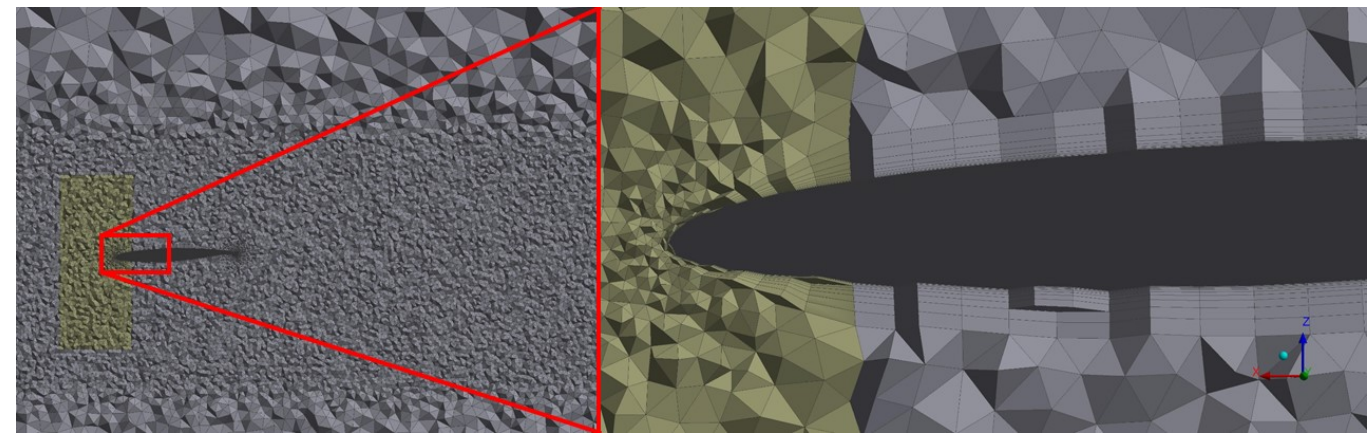

(b) Y-axis slice of the mesh

Figure 4.2: Slices in mesh for observation of inflation layer generation in Ansys

Upon multiple meshing iterations, problems were found with the continuity of the mesh near the trailing edge and the wingtip of the model. To improve the quality in these areas, a face sizing command was utilized to specify a locally smaller cell size to override the global settings. This was also the case for areas of the wing close to the fuselage. Because of the tight corners of the model, cells placed in these areas were becoming skewed beyond what the solver was able to handle. An example of this refinement can be seen in Figure 4.3. Furthermore, to ensure that the cells along the wing were fine enough to capture additional flow metrics, and edge sizing was placed on the leading edge of the wing that propagated along the wing surfaces. This caused the cells on the bottom and top surface of the wing to be further reduced in size from the global setting, and can be viewed in Figure 4.2.

The meshes produced for the 0 degree and 5 degree angle of attack simulations were then saved and imported into fluent for processing. It should be noted that only the 0 degree angle of attack case was simulated for 25 and 35 meters per second, while the 5 degree 


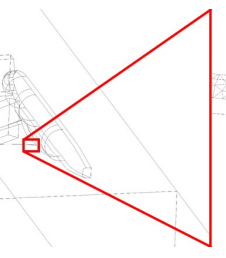

$\therefore$

Figure 4.3: Region of cell refinement to improve cell skew and mesh quality

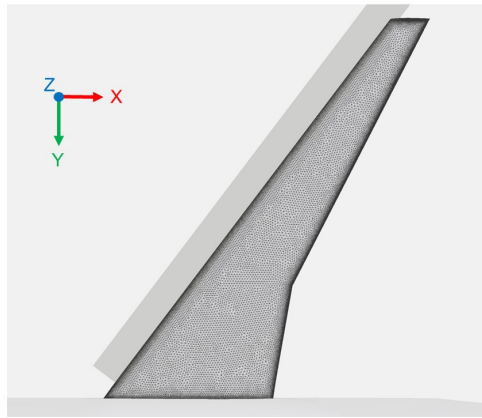

(a) Surface mesh

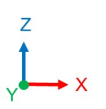

(b) Fluid domain mesh

Figure 4.4: Final mesh views for the 0 degree angle of attack simulation

angle of attack case only underwent a 25 meter per second simulation. The final mesh sizes and major statistics for each model are shown in Table 4.1.

Table 4.1: Mesh statistics for both modeled cases

\begin{tabular}{|c|c|c|}
\hline & 0 Angle of Attack & 5 Angle of Attack \\
\hline Elements (Millions) & 31.57 & 37.64 \\
\hline Nodes (Millions) & 7.62 & 8.73 \\
\hline First Layer Height (mm) & 0.004 & 0.004 \\
\hline Skew (Avg) & 0.22529 & 0.2463 \\
\hline Element Quality (Avg) & 0.70856 & 0.6814 \\
\hline
\end{tabular}

\subsubsection{Processing}

Processing of the fluid mesh was done as a Reynolds Averaged Navier Stokes (RANS) simulation using Fluent. The solution utilized the SIMPLEC solver due to its ability to better resolve the simulation than the SIMPLE solver. Second order momentum equations were also utilized to be consistent with the second order function associated with the pressure discretization at cell faces. Furthermore, the fluid domain was assumed to have 
a $2 \%$ turbulence intensity to match the approximate cal poly tunnel turbulence intensity, with a length scale of $4 \mathrm{~mm}$, based on $2 \%$ of the MAC. The entire domain was assumed to be turbulent flow with the exception of the laminar zones discussed in Chapter 2. The simulations were run at 35 and 25 meters per second at 0 angle of attack, and 25 meters per second at 5 degrees angle of attack. The residuals were tracked and plotted actively during the simulation to verify when convergence was reached, as no hard limits on residual convergence were set. Convergence was considered achieved when the residuals did not change by more than $1 \mathrm{e}-2$ over 100 iterations. The residuals for each configuration are shown in Figure 4.5.

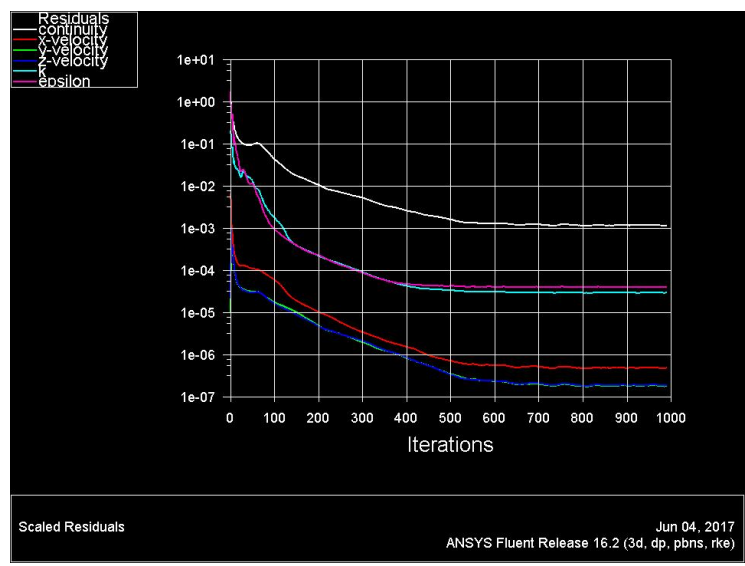

(a) $25 \mathrm{~m} / \mathrm{s}$ residuals

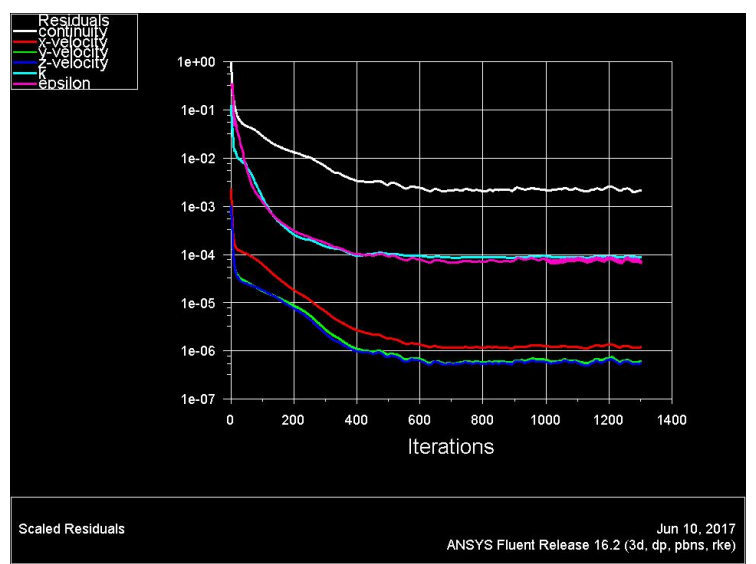

(b) $35 \mathrm{~m} / \mathrm{s}$ residuals

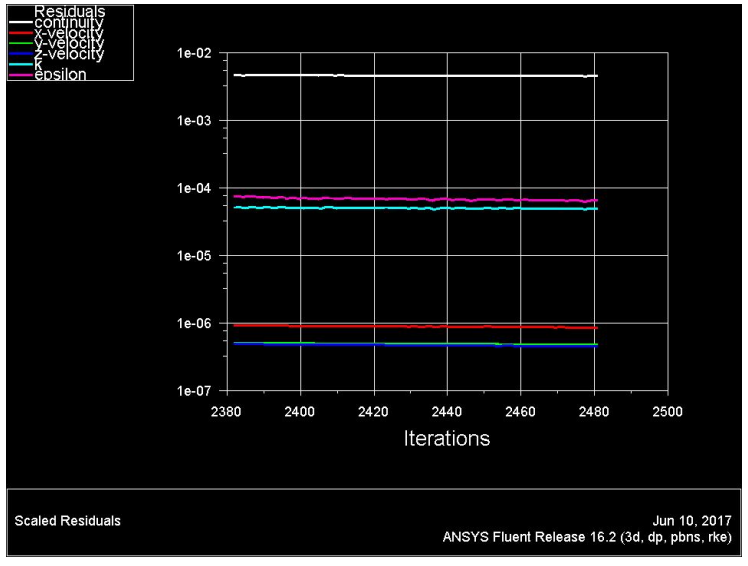

(c) 5 aoa residuals

Figure 4.5: Residual results for each Fluent case 
Once solution convergence was confirmed, a mesh independence study was performed to ensure that the fluid domain mesh was refined enough to not influence the results of the simulation. This was done by producing a coarse, medium, and fine mesh for the 0 angle of attack configuration, and observing changes in lift and drag coefficient. The mesh sizes and percent change in aerodynamic coefficients are shown in Table 4.2. Looking at the table, we see that the lift coefficient between meshes encounters less than $1 \%$ change. However, the drag coefficient is a bit more significant at over $1 \%$ change between the fine and medium mesh. This may be more of a cause of poor mesh quality in regions close to the junction between the wing and fuselage rather then the mesh size itself. While not ideal, this study is not overly concerned with the overall drag numbers of the model, and more concerned with a specific location in the wake and the lift force along the wing.

Table 4.2: Mesh convergence for the $25 \mathrm{~m} / \mathrm{s}, 0$ angle of attack configuration; percent difference based off deviation from finest mesh

\begin{tabular}{|c|c|c|c|}
\hline & Coarse & Medium & Fine \\
\hline Elements (Millions) & 15 & 20 & 31 \\
\hline$C_{D} \%$ difference & 0.8258 & 1.1790 & - \\
\hline$C_{L} \%$ difference & 0.0165 & 0.0271 & - \\
\hline
\end{tabular}

Because of the turbulence assumption for the fluid domain, a turbulence model for the simulation needed to be selected. The mesh first layer height for all simulations was made such that a $y^{+}$of 1 was produced prior to the simulation, which is necessary for some of the turbulence models in Fluent. The $y^{+}$metric is a number that describes how coarse or fine a mesh is near a wall in the fluid, and is based on the local Reynolds number of the flow. The SST k- $\omega$ and Realizable k- $\epsilon$ turbulence models were chosen for investigation due to their ability to resolve the flow in this range. Both models were used to solve the simulation, and observations of their residuals and final answers were noted. It was found after solving that the SST model predicted flow separation along most of the wing surface, and is shown in Figure 4.6.This phenomena occurred for multiple meshes and configurations, and was therefore discarded as the appropriate turbulence model for the 


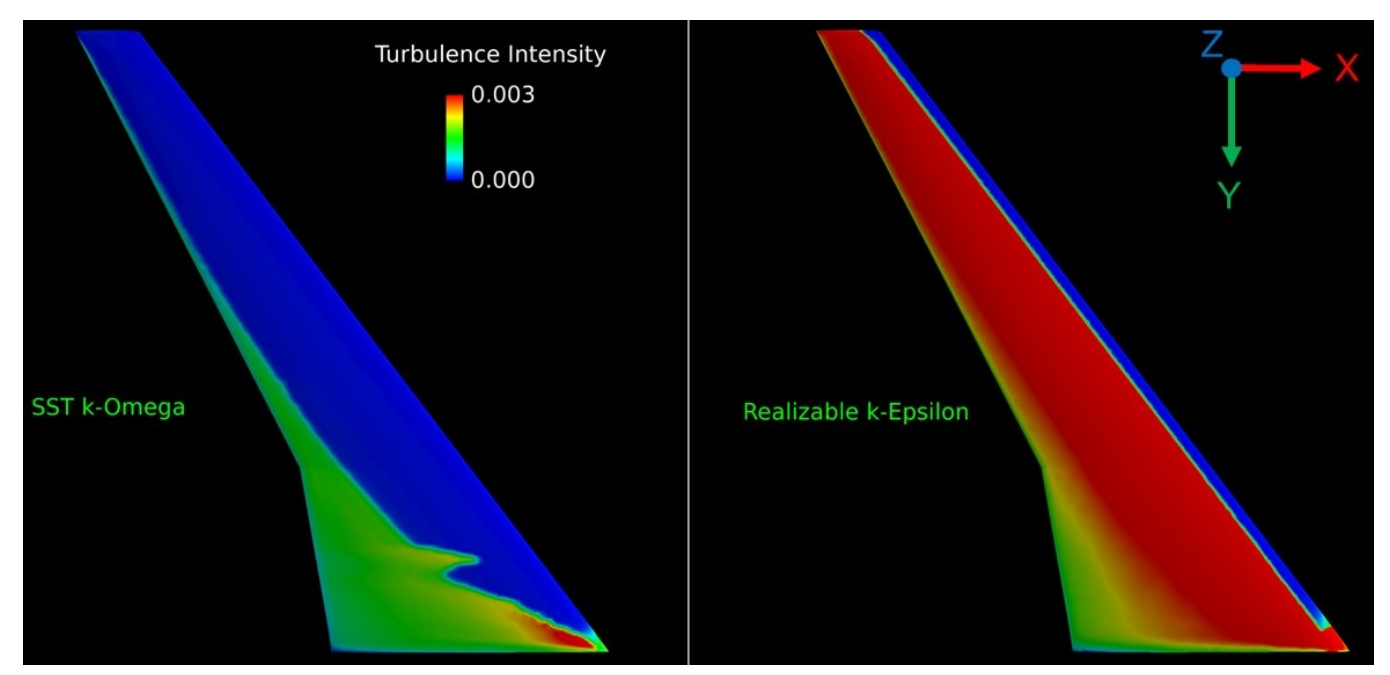

(a) Contour of turbulence intensity, indicating separation zone present in SST k- $\omega$ turbulence model compared to the Realizable k- $\epsilon$ model

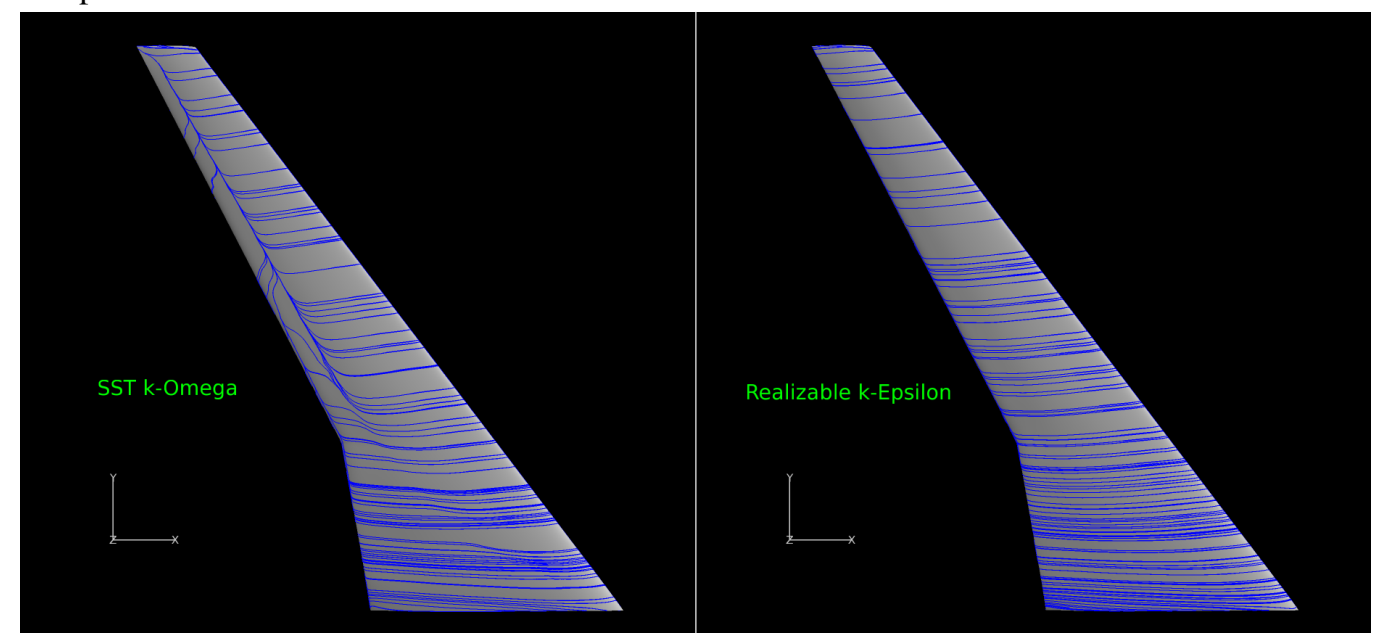

(b) Comparison of the surface streaklines of the SST vs. Realizable turbulence models

Figure 4.6: Comparison of turbulence models for Low Reynolds number transition in FieldView

simulation. This was justified due to a flow visualization experiment discussed in Section 5.1 that confirmed that flow was attached to the wing for all configurations considered. Therefore, a realizable k- $\epsilon$ turbulence model with pressure gradient effects and an enhanced wall function was utilized for all configurations. 


\subsection{Finite Element Analysis}

The solid domain used for FEA utilized the model produced during manufacturing CAD design. This model was meshed and solved in Ansys Mechanical to determine the nodal deflection of each point in the mesh of the model under the loads imported from the CFD simulation, with emphasis on determining the z-axis deflection at the wingtip. However, additional simplifications and assumptions were made prior to import into Ansys Mechanical for meshing.

1. The combined deflection of the tunnel mounting assembly and the fuselage is small enough such that they have little to no effect on the overall wing deflection, and can be removed from the model. This is based on prior calculations indicating a maximum deflection of less than 0.01 millimeters for both the fuselage and the tunnel mounting assembly.

2. The connections between the tipwing, midwing, and basewing act as bonded pairs, and therefore transfer loads and deflections unimpeded by the material properties of the epoxy compound used. Because the construction of the wing used a bonding scheme rather than a bolting assembly, this assumption is correct.

3. Both materials simulated behave as homogeneous and isotropic materials, whose effective density is based on the inherent density of the material and the \% infill used during printing. While the assumption is not truly reminiscent of the behavior of FDM parts, it is necessary to obtain a sufficient solution in a short amount of time and computing resources when compared to an anisotropic solution build up.

4. The simulation behaves as a linear-elastic loading scenario. This assumption is based on the understanding that the loads imparted on the wing are not enough to cause 
plastic deformation, and that the experiment was not ran enough times to incorporate fatigue.

With these assumptions, FEA solutions were produced for each loading scenario and for each Nylon wing, resulting in 6 total FEA cases. The material properties used during the simulations are listed in Table 3.1. Because each wing used a 50\% infill command during printing, the effective density for the simulations are $50 \%$ of the density listed in Table 3.1.

\subsubsection{Meshing}

Prior to meshing, the connections needed to be specified in Ansys. This involved selecting the faces on the basewing that are bonded to the midwing and vice versa and specifying their contact type. The same process is applied to the midwing and tipwing contact region. As mentioned previously, a bonded contact was assumed for the assembly pieces. Ansys also allows the user to make additional inputs into the FEA solver to better describe the behavior of the connection. Primarily, the Ansys defaults were used for contact calculation with the exception the "behavior" and "formulation" settings.

Behavior was specified to be asymmetric and formulation was selected to utilize MPC (Multi-Point Constraint). Asymmetric behavior, also known as "one-pass contact", specifies one part as the "contact" and one part as the "target". Deflections applied at the contact region are passed from the contact to the target only, and is more efficient in solving simple models. However, as a verification that the model was still accurate, simulations were run with 2-pass contact. The results yielded less than $1 \%$ change in $\mathrm{Z}$ deflection, which led to 1-pass contact being used for further simulations. For the two connections in the FEA solution, the female connection was specified as the contact, while the male connection was the target, and can be seen in Figure 4.7. The MPC formulation allows for a truly 


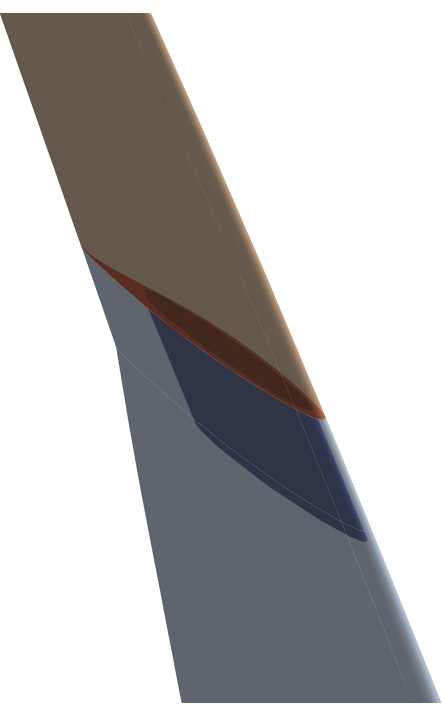

(a) Contact between the basewing and midwing

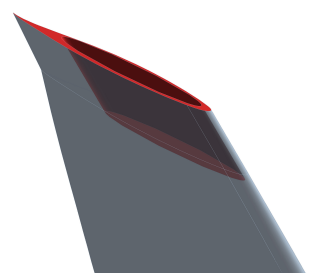

(b) Basewing contact faces

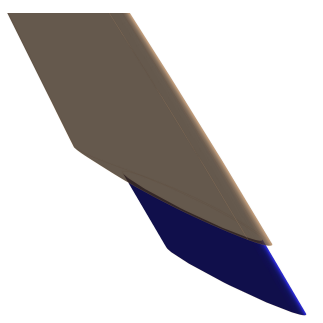

(c) Midwing target faces

Figure 4.7: Example of contact region for the basewing to the midwing

linear solution with no penalty on the model for penetration or separation of parts from one another.

The mesh constructed for the assembly utilized a conformal, unstructured mesh, and is consistent across all cases. Similar to meshing for CFD, many of the program defaults were selected in lieu of additional editing. The global mesh settings that were altered for the FEA simulation are listed in Appendix B. Additional refinement was necessary for the leading edge, trailing edge, and wingtip due to the global settings producing too coarse of a mesh in these areas to properly define the shape of the model. The element sizes in these regions were constrained to be a size of $8 \mathrm{e}-004 \mathrm{~m}$ to resolve the mesh to a proper refinement. The result of these mesh settings lead to a mesh size of 1.11 million elements, and can be viewed in Figure 4.8.

Upon construction of a mesh, loads could then be imported onto the model from the CFD simulations. The process first involved identifying the node locations of pressure 


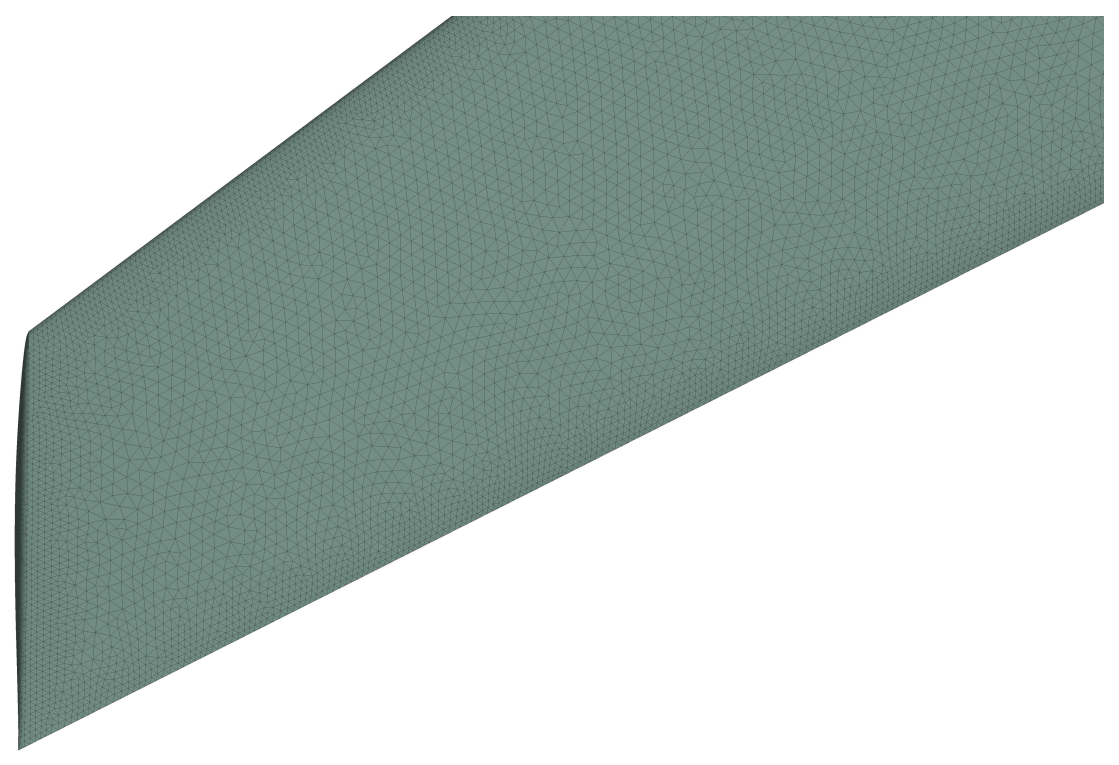

(a) Zoomed view of top
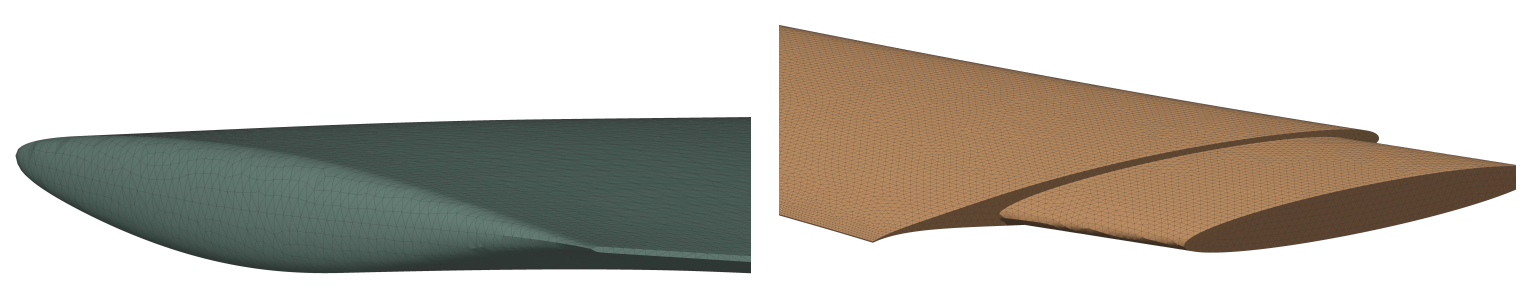

(b) Wingtip and trailing edge mesh

(c) Contact mesh on midwing

Figure 4.8: Mesh views for the FEA simulation

loads on the CFD mesh for each node in the CFD mesh. The nodal locations of these pressure forces are then located onto the mesh to be used for the FEA simulation. However, it is incredibly unlikely that the exact same node locations will be present for both CFD and FEA meshes, especially for an unstructured mesh. Therefore, Ansys went through a process of interpolating the nodal pressures from the CFD onto the nodes of the FEA mesh. Additionally, model faces needed to be specified to ensure that Ansys located the pressures appropriately. This involved selecting where the laminar zones were, the trailing edge, etc. Once selected, the pressures taken from each steady state CFD case could be loaded as a 
different loading case for FEA simulations. The result of the 25 meter per second case loading scenario is shown in Figure 4.9.

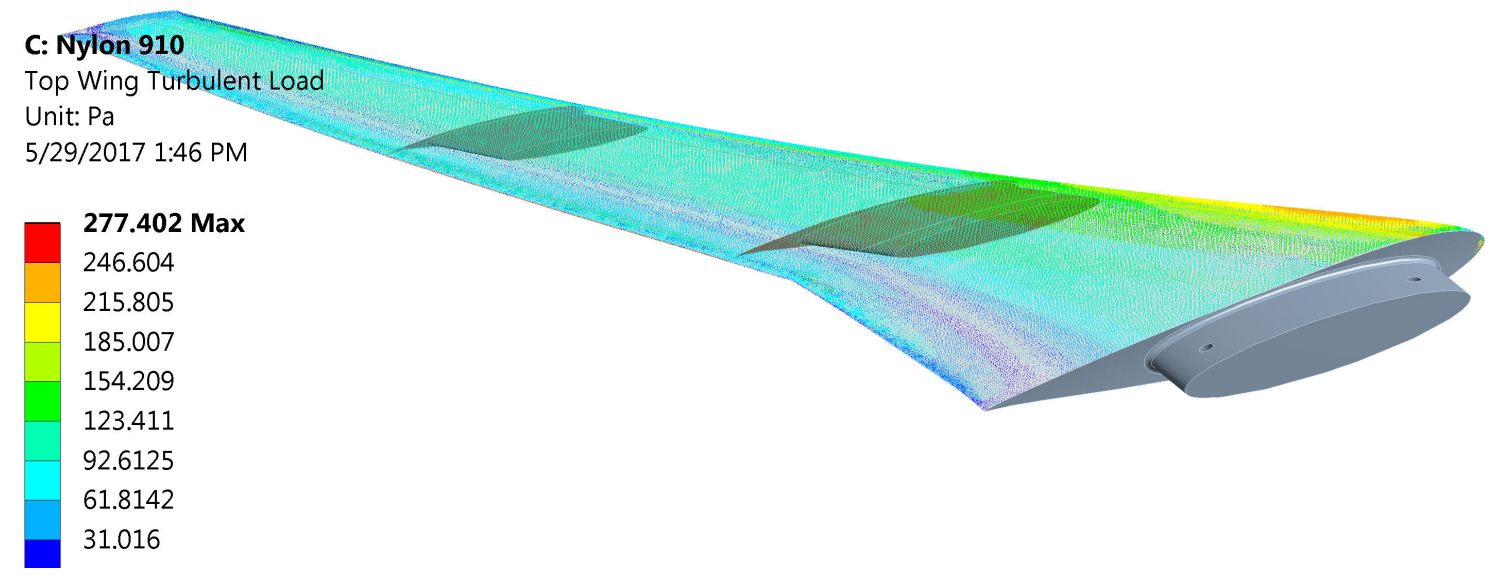

Figure 4.9: Pressure loading vectors at each node for the $25 \frac{\mathrm{m}}{\mathrm{s}}$ loading case

\subsubsection{Processing}

A direct solver was used due to the assumptions made for the FEA simulations, which made the solution relatively simple. Additionally, all other program defaults for Ansys were utilized with the exception of the number of time steps. Because both the 25 and 35 meter per second load cases involved the same geometric model, they could be solved in one simulation. To apply this, 3 different steps were used to simulate the loads individually. First, the solver applied the loads imported from the 25 meter per second CFD case. The second step involved unloading the wing back to its original state. Finally, the third step loaded the 35 meter per second case to conclude the simulation. This process could not include the loads taken from a 5 degree angle of attack case because of the geometric differences between the models.

The extrusion of the basewing that slots into the tunnel block acted as the support for the solver, and can be seen in Figure 4.10. Because of how the wing sits in the tunnel block, it could not rotate about any of the axes. Furthermore, the tunnel block prevents translation 
in the $\mathrm{x}$ and $\mathrm{z}$ axis. The translation constraint in the $\mathrm{y}$-axis is prevented by the 2 shear pins that pass through the entire model assembly. Because of these constraints, the basewing extrusion utilized a fixed support for the simulation.
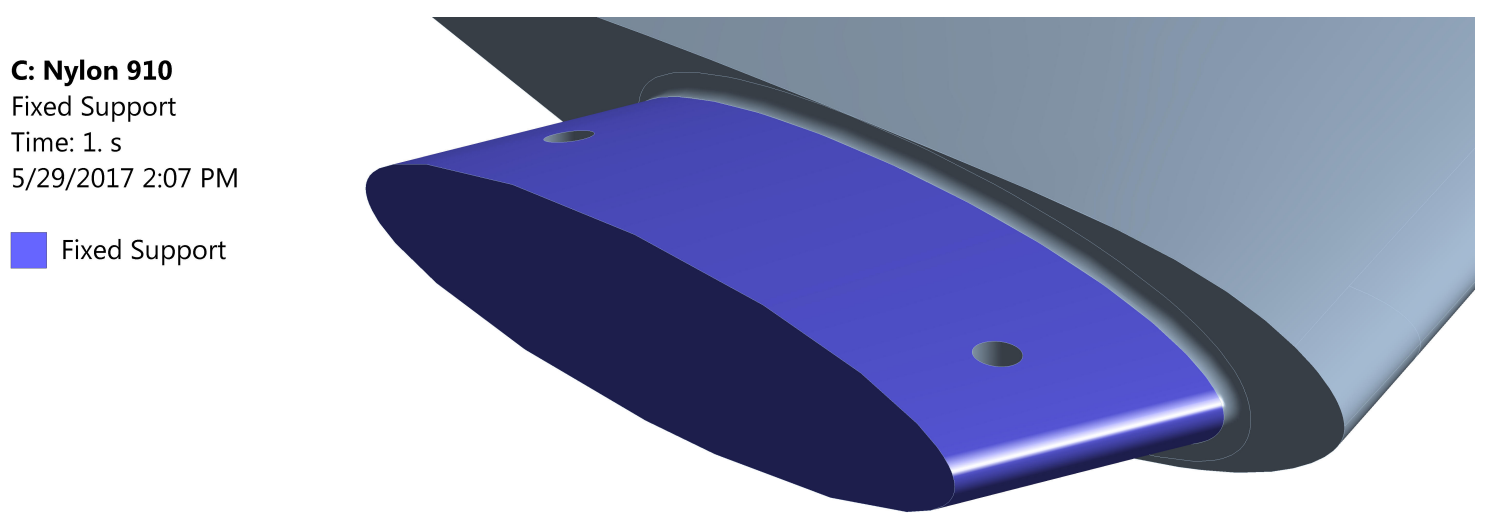

Figure 4.10: Fixed support for all loading cases

Prior to solving the solution, the analysis settings of Ansys were altered to include two time steps in the solution. The first time step, from 0 to 1 second, applied a gravitational load on the wing. Once the gravitational load had been applied, the loading scenario from CFD was then applied from 1 to 2 seconds during the second step. This would ensure that the delta deflection from gravity to wind on would correlate to the deflection measurements taken from videogrammetry.

To verify the integrity of the mesh, multiple sensitivity cases were explored to determine how much of an effect the mesh had on the FEA solution. The first sensitivity study involved a mesh refinement, identical to that done in CFD. 3 separate meshes were generated, each varying in the number of elements present, and the delta $\mathrm{z}$ deflection was recorded for each mesh. Table 4.3 shows the percent difference in delta $\mathrm{z}$ deflection for the 5 degree angle of attack simulation, as this was the most extreme loading case scenario and the most likely to have errors due to mesh size and quality. 
Table 4.3: Mesh convergence for the 5 degrees angle of attack configuration; Percent difference based off deviation from finest mesh

\begin{tabular}{|c|c|c|c|}
\hline & Coarse & Medium & Fine \\
\hline Elements (Thousands) & 286 & 348 & 472 \\
\hline$\Delta Z \%$ difference & 2.06 & 0.26 & - \\
\hline
\end{tabular}

Because the difference between the finest and medium mesh was less than $1 \%$, it was assumed that the solution was independent of the mesh size, and that further solving could continue. For reassurance purposes, the finest mesh was used for all future FEA cases.

The next validation necessary was mesh quality. The meshes produced for FEA consisted of primarily 3D tetrahedral and wedge type elements. These are often considered poor choices for FEA due to the elements causing incorrect deflection values in bending due to locking $^{[5]}$. To verify the change in deflection due to element type, a structured mesh using 3D Hexehedral elements was produced. The mesh consisted of 19,072 elements, and can be seen in Figure 4.11

Upon solving the mesh for the 5 degree angle of attack case, it was found that the delta $\mathrm{z}$ deflection of the model had only a $1.2 \%$ change from the finest tetrahedral mesh, it was determined that the element type had little effect on the outcome of the simulation. However, because the hexehedral mesh produced a smaller density mesh, and had overall better quality elements, it was used for the remainder of the simulations. This drastically improved load mapping time, solving time, and computer resources.

Finally, to asses the uncertainty in material response, a material properties sensitivity study was performed. This involved increasing the Young's Modulus and Poisson's ratio of the material by 10,20 , and 30 percent and recording the delta $\mathrm{z}$ deflection value. If the solution was independent of the mesh, then the delta $\mathrm{z}$ changes for each increase in the material properties should be linear. The results of this study are shown in Table 4.4 


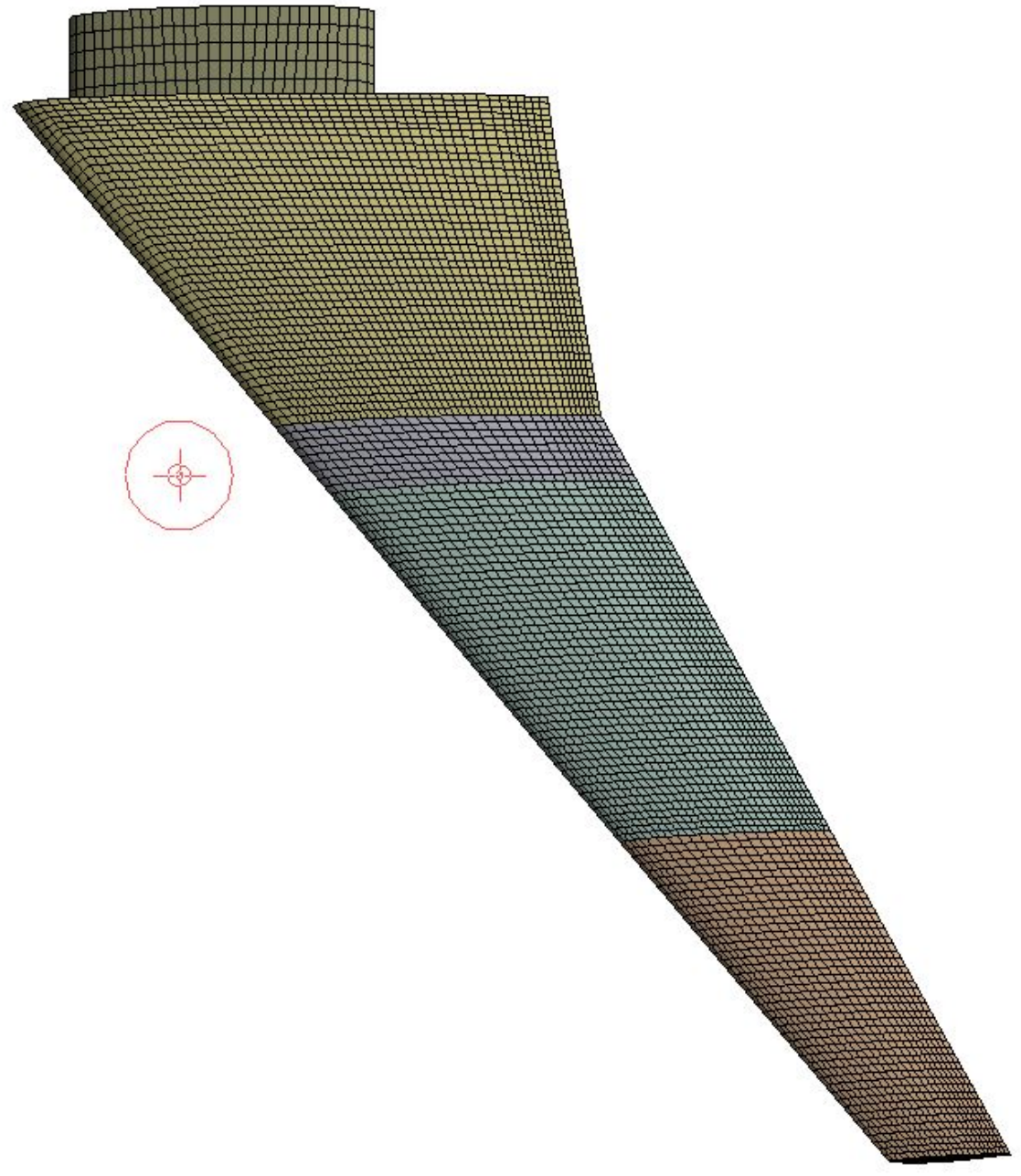

Figure 4.11: Structured mesh for FEA element type sensitivity analysis

The results in Table 4.4 indicate a linear slope of approximately 0.7075 percent change in deflection for every percent change in material properties. Because the trend is linear, with an $R^{2}$ value of 0.9978 , it is assumed that the mesh does not influence the solution outcome. 
Table 4.4: Material properties sensitivity analysis results

\begin{tabular}{|c|c|c|c|c|}
\hline & Baseline & $\mathbf{+ 1 0 \%}$ & $\mathbf{+ 2 0 \%}$ & $\mathbf{+ 3 0 \%}$ \\
\hline Young's Modulus (MPa) & 665 & 731.5 & 798 & 864.5 \\
\hline Poisson's Ratio & 0.31 & 0.34 & 0.372 & 0.403 \\
\hline$\Delta Z$ \% difference & - & 9.71 & 17.36 & 23.86 \\
\hline
\end{tabular}




\section{EXPERIMENT}

\subsection{Setup and Preliminary Testing}

Prior to obtaining metric data on the aerodynamics or structures of the model, it was necessary to ensure that the model would endure the speeds proposed in the research without causing harm to the people involved, the facility, or the model itself. A check load was performed on the model to ensure it would survive speeds up to 35 meters per second. This process was simplified to simply bringing the wind speed up to 50 meters per second, and tethering the model to the floor using safety wire. This way, if the wing failed, a tunnel shutdown could be implemented before further harm came to the facility or its inhabitants. Figure 5.1 displays the tethering process used to secure the wings. Each wing successfully survived at 50 meters per second. However, due to excessive wing tip vibrations, it was determined that speeds above 35 meters per second for 0 degrees angle of attack or 30 meters per second for 5 degrees angle of attack were unsafe.

In order to fix boundary layer transition on the wing model, a trip strip was placed on the wings at a location of approximately $7 \%$ of the MAC, aft of the leading edge, thus matching the transition point used in the CFD simulations. Using methods established by previous experiments and processes $^{[4],[11]}$, P80 grit sandpaper with an adhesive backing was used to trip the boundary layer. Figure 5.2 gives a view of the trip strip used on the wings.

In order to verify that the trip strips were transitioning the boundary layer, a flow visualization experiment with oil flow was performed. The process involved placing motor oil mixed with white pigmentation powder on the top surface of the wing, before and after the trip strip. With the wind on, the oil will streak across the top surface of the wing if the flow is attached, and it will begin to form pools of fluid where the flow separates. The flow 


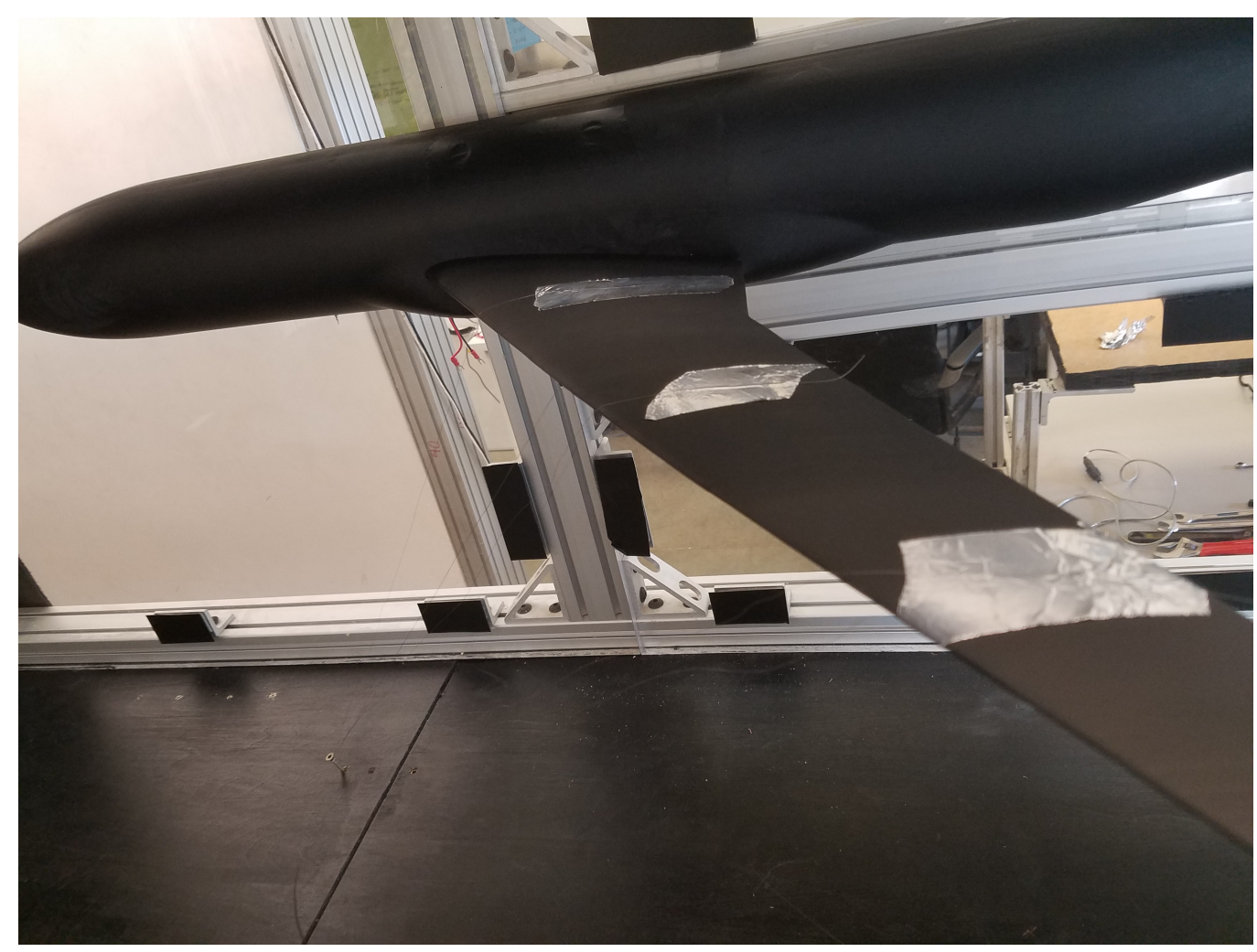

Figure 5.1: Tethering of the ABS wing for pre-test check loads

visualization experiment was performed on the wings at 25 meters per second and 0 degrees angle of attack. Due to the viscosity of the motor oil compound, the streaklines required approximately 10 minutes to fully develop over the wing, at which time it was noted that the oil was no longer moving across the wing surface. Figure 5.2 shows the results of this experiment. By looking at Figure 5.2, it can be seen that the flow is attached to the wing until it reaches the trailing edge of the wing, where it separates. This separation is also present near the junction of the wing and fuselage, which is likely due to the interaction of the boundary layers of the wing and fuselage. Based on previous experiments performed with the CRM, it was visually concluded that the trip strips were performing as intended, and that turbulent flow was achieved ${ }^{[11]}$. 


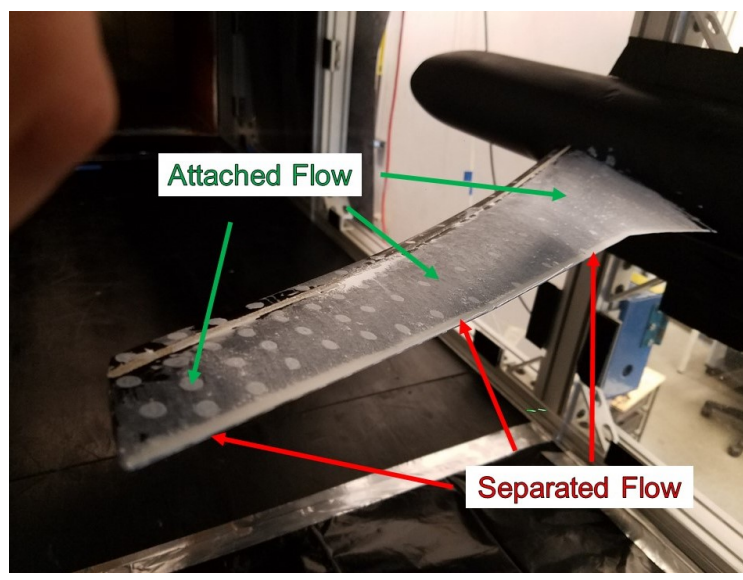

(a) Wingtip aft view

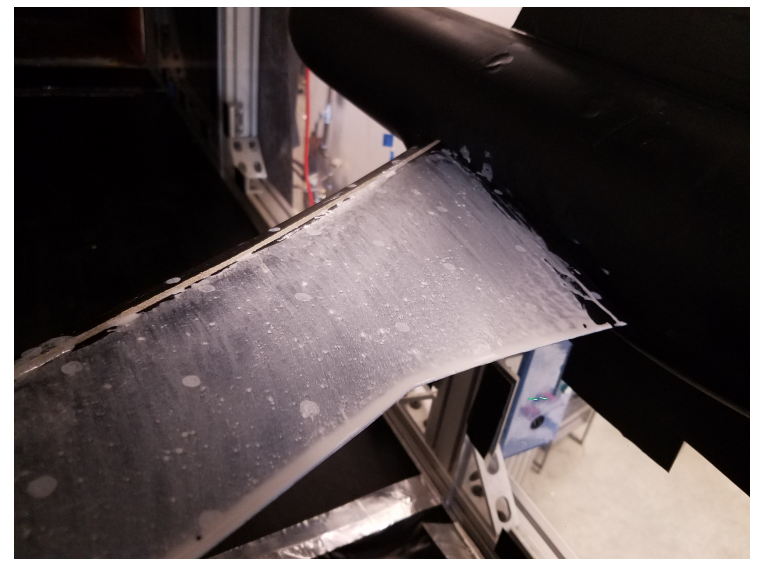

(b) Wing root aft view

Figure 5.2: Oil flow attachment and separation for Cal Poly CRM

\subsection{Wake Rake}

A 3-axis traverse was used to make precision movements of a 5-hole probe placed in the wake of the wings for each configuration case. The probe was placed such that the tip of the stagnation port was in a plane that was 50.8 millimeters ( 2 inches) aft the wingtip trailing edge for all measurements. This prompted an x-axis location of the traverse at $397 \mathrm{~mm}$ from the downstream limit switch, where the x-axis "home" point is located. Once in this location, z-axis sweeps of the probe in $1 \mathrm{~mm}$ increments were performed at 3 different $\mathrm{y}$-axis locations along the wing span to observe the momentum loss of the wing at the tipwing, midwing, and basewing. The starting and ending locations of the $\mathrm{z}$-axis movement were dependent on the size of the wake. The sweeps began and ended at instances where the difference between the probe stagnation pressure and the tunnel stagnation pressure were either equal or within $5 \%$ of frestream. When at these locations, it was assumed that the probe had gone outside of the wing wake, and additional data was no longer necessary. The starting and ending locations for the traverse for each axis and configuration are shown in Table 5.1. It should be noted that all z-axis distances given in Table 5.1 are in millimeters from tunnel centerline. Furthermore, the $\mathrm{z}$ limits listed in Table 5.1 may not represent the 
exact limits of the wing wake, which can vary by a few millimeters due to the vibration of the wing.

Table 5.1: $\mathrm{Y}$ and $\mathrm{Z}$ axis locations of the traverse for the wake rake; Distances are measured from tunnel centerline

\begin{tabular}{|c|c|c|c|c|c|c|c|}
\hline & & \multicolumn{3}{|c|}{ Nylon 618} & \multicolumn{3}{|c|}{ Nylon 910} \\
\hline & y (\% Span) & $89.75 \%$ & $53.16 \%$ & $16.57 \%$ & $89.75 \%$ & $53.16 \%$ & $16.57 \%$ \\
\hline \multirow{2}{*}{$25 \mathrm{~m} / \mathrm{s}$} & $\mathrm{z}$ minimum $(\mathrm{mm})$ & 56 & 20 & -6 & 56 & 23 & -5 \\
\hline & $\mathrm{z}$ maximum $(\mathrm{mm})$ & 69 & 40 & 16 & 69 & 41 & 20 \\
\hline \multirow{2}{*}{$35 \mathrm{~m} / \mathrm{s}$} & $\mathrm{z}$ minimum $(\mathrm{mm})$ & 57 & 22 & -6 & 56 & 24 & -5 \\
\hline & $\mathrm{z}$ maximum (mm) & 71 & 42 & 17 & 67 & 42 & 20 \\
\hline \multirow{2}{*}{5 AoA } & $\mathrm{z}$ minimum $(\mathrm{mm})$ & 68 & 16 & -25 & 68 & 18 & -24 \\
\hline & $\mathrm{z}$ maximum (mm) & 80 & 35 & 5 & 81 & 35 & 6 \\
\hline
\end{tabular}

Figure 5.3 shows the wake rake at the desired $\mathrm{x}$ axis position behind the wing. Due to the aft end of the fuselage blocking the traverse from moving closer to the wingtip along the $\mathrm{x}$-axis, The probe had to be extended forward to reach the wake position desired. A 3D printed part made of T-Glase was produced to attach to the z-axis arm of the traverse and attach to the probe. The inside of the part where the probe is mounted is lined with both felt and tape to provide a better seal to the probe as well as reduce vibrations caused by the wind. Furthermore, a leg extends from the bottom of the z-axis traverse to the floor to provide the traverse strut additional support, thus reducing probe position error due to cantilever beam deflection.

The 5-hole probe and the static port pressure differentials from the tunnel static pressure were measured using a Scanivalve ZOC 33 module and an ERAD 4000. The probe pressure ports and the tunnel dynamic pressure were measured using diaphragms with a 10 inch water column differential limit. The information from these two units was then transferred to a Labview program for organization and analysis. The ERAD measured pressure data at a rate of approximately $15 \mathrm{~Hz}$, and collected 200 data points per z-axis location. The pressure data was then saved as a .mat file for post-processing in Matlab. 


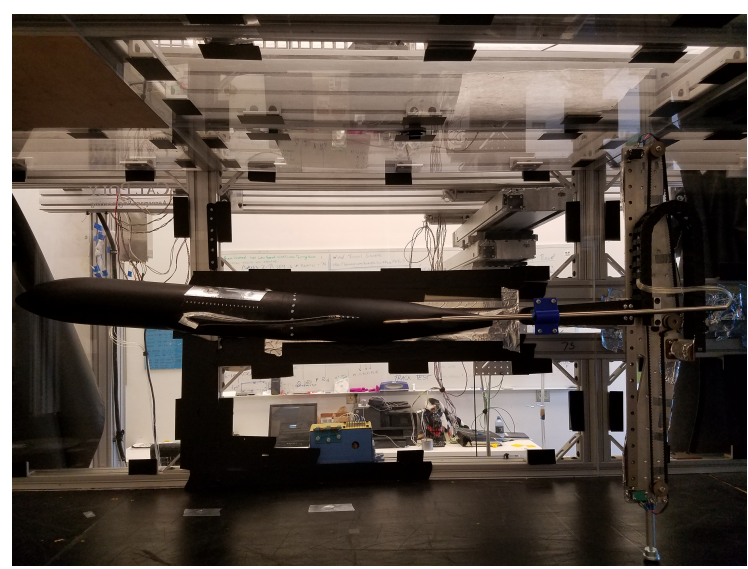

(a) Far view of wake rake setup

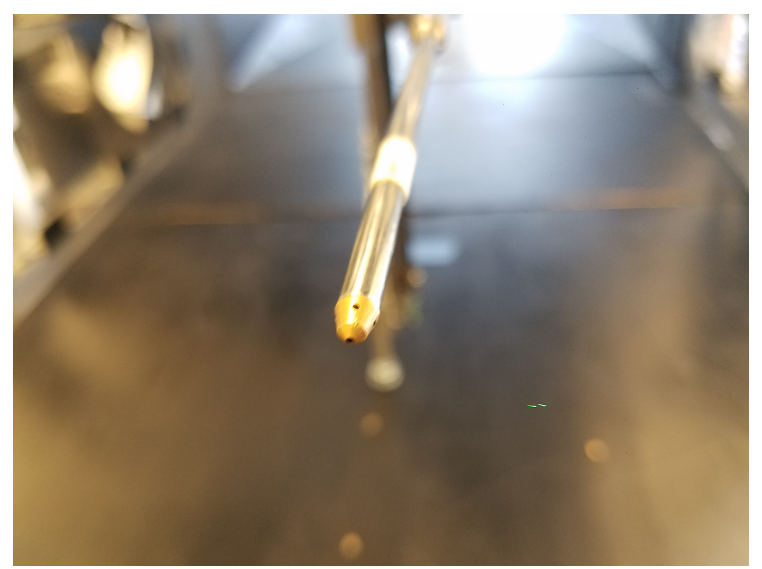

(b) Close view the probe

Figure 5.3: Various views of the 5-hole probe used in the wake study

\subsection{Videogrammetry}

In order to measure the deflection of each wing during testing, the videogrammetry method was selected such that the data could be obtained without intruding into the flow or the model. The method involved painting dots on the wings that could act as tracking points for the cameras, seen in Figure 5.4. The videos obtained by the cameras while the wind was both off and on could be uploaded to Matlab, where the vision toolbox utilized an algorithm to track dot movement in pixels during the video. This was then converted to inches by calibrating a pixel per inch for each camera view.

\subsubsection{Hardware Settings}

Three cameras were used to obtain the deflection data for each wing during each run. The $\mathrm{x}$-axis camera was placed inside the tunnel, 3 inches aft of the end of the test section. The $y$-axis camera was placed outside the door to the test section such that it was in line with the wing. The z-axis camera was placed on the roof above the wing. All three cameras were set to the highest resolution possible at 30 frames per second. 
The most useful camera for this test was the y-axis camera, as it had the best view of the wingtip and was not influenced by the wind tunnel being on or off. It also used a $135 \mathrm{~mm}$ lens, which eliminated a lot of distortion in the video due to perspective. This camera, therefore, acted as the master during data analysis as it had the most trustworthy results. Next was the $\mathrm{x}$-axis camera because of the wings tendency to deflect in the z-direction, offering the ability to track the points along the wing's span. However, because the camera had to be placed inside the tunnel to get a view of the wing, it was subject to its own deflection and vibrations during the experiment. While this can be corrected using datum points, as seen in Figure 5.4, it does introduce the potential for error in the analysis. Finally, the z-axis offers the least amount of fidelity in the results because of the wings tendency to deflect towards the lens. Another reason is because the $\mathrm{z}$-axis used a wide-angle lens to capture the entire wing surface. While being on top of the tunnel with a wide-angle lens allows this camera to view the most dots, it offered little in terms of being able to accurately analyze the movement of those points. However, this camera did act as a sanity check using information obtained from both the $\mathrm{x}$ and $\mathrm{y}$-axis cameras. The views that each camera had of the wing surface can be seen in Figure 5.4

\subsubsection{Software Settings}

Once the videos of each camera were obtained for the Nylon wings at each run schedule, the

videos were edited in Adobe Premiere. The first step was to shorten the length of each video to only what was necessary to get a wind off and wind on reading to reduce the computation time required by the computer. This resulted in each video being approximately 1 minute long. Furthermore, each video was edited such that all three cameras were in sync up to a fidelity of 1 millisecond. Due to hardware and software limitations during the experiment, the cameras could not be triggered to begin and end capturing simultaneously. Thus, verbal cues were given during the experiment to signal that data taking should begin and end. 


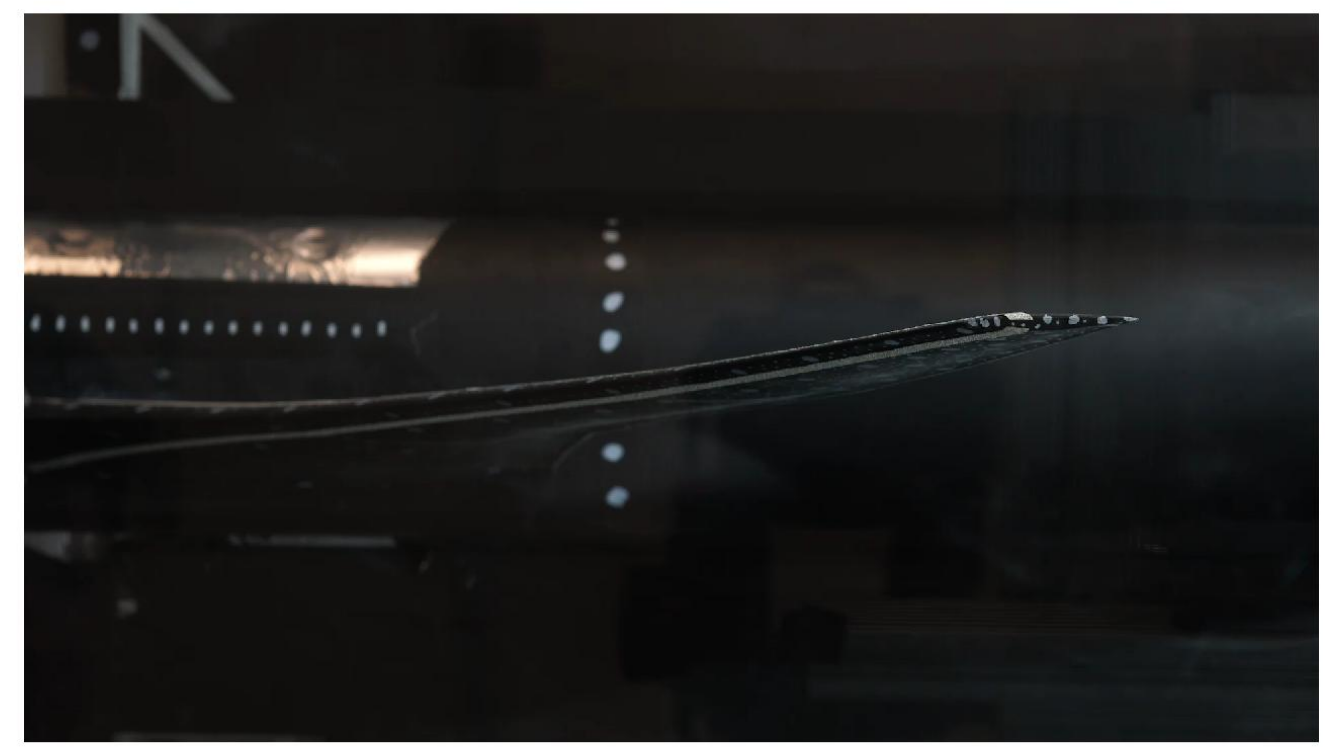

(a) View of the y-axis camera

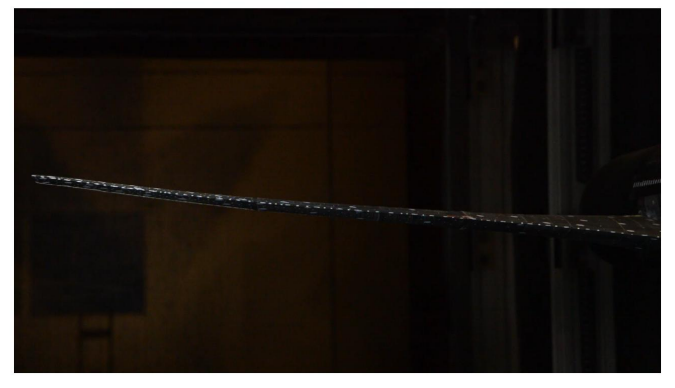

(b) View of the x-axis camera

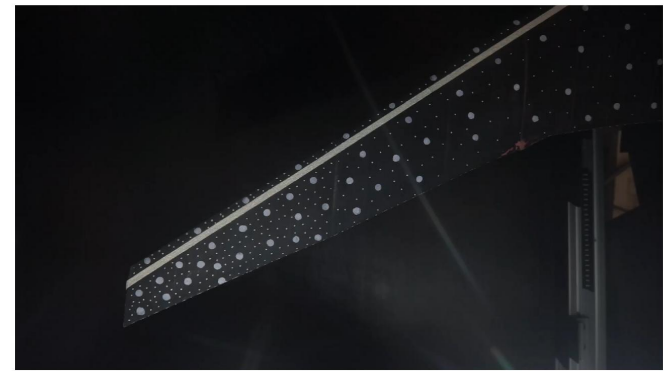

(c) View of the z-axis camera

Figure 5.4: View that each camera has of the wing configuration during testing

These verbal cues served as the cutoff points for each video to ensure that each camera's exposure during the experiment began and ended simultaneously.

Once the videos were clipped, synchronized, and exported as .mp4 files, they were uploaded to Matlab for processing. The first step involved determining which dots to track and their location on the screen. For the y-axis, the dots on the wingtip were selected along with 4 datum dots on the fuselage, as depicted in Figure 5.7. The pixel location of these dots was determined by hovering over their approximate CG location, and tabulating the $\mathrm{x}$ and y coordinates of that pixel on a 1920x1080 pixel screen. 


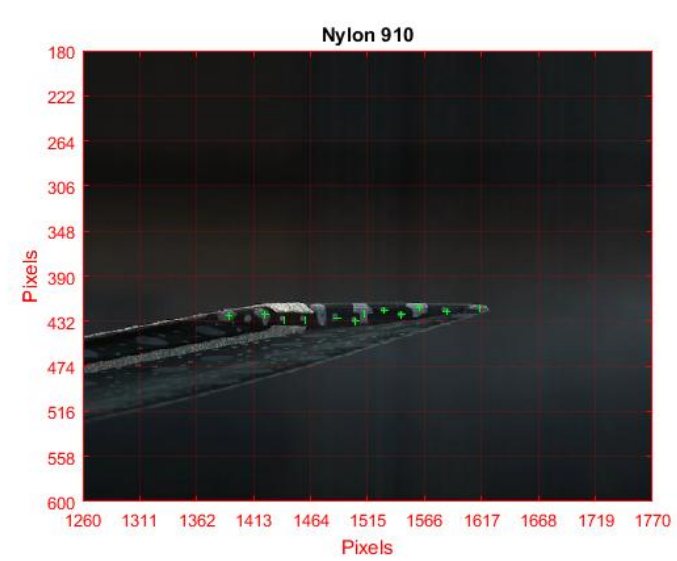

(a) Nylon 910 at 0 degrees angle of attack, wind off

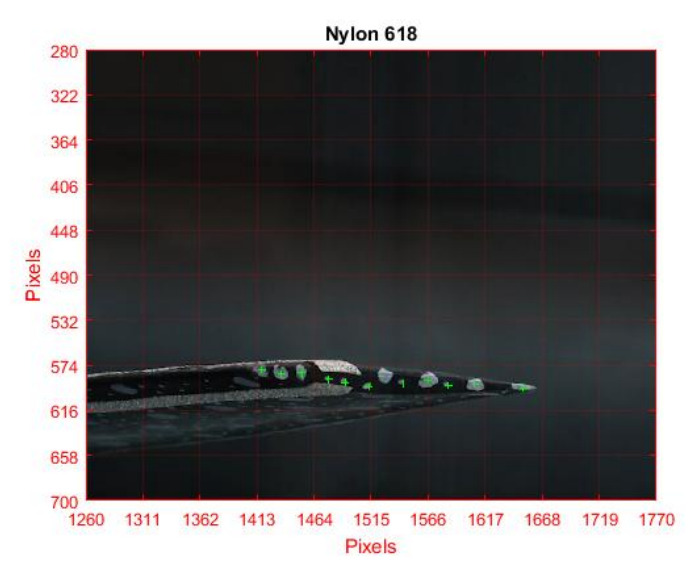

(b) Nylon 618 at 5 degrees angle of attack, wind off

Figure 5.5: Dot tracking initialization in Matlab for 2 configurations

The dots for the $\mathrm{x}$-axis camera involved the same process, but focused instead on dots located along the trailing edge of the wing, with even more emphasis placed on dots near the wingtip. The $\mathrm{x}$-axis camera also used the dots along the fuselage to act as a datum for the experiment. These datum points were much more important for this camera due to the vibration and movement the camera experienced by being placed inside the wind tunnel. The pixel movement of the datum points caused by camera movement was subtracted out of the movement from the other dots to compensate for camera vibration and movement. Because of the large potential for error in this method, the $\mathrm{x}$-axis camera only serves as a validation point for the y-axis camera values. Figure 5.6 shows the dots being tracked by the $\mathrm{x}$-axis camera.

A loop execution in Matlab took the initial pixel locations of the dots for both cameras, and then tracked their movement during the test for each frame of the video. The movement of each dot, in pixels, was stored in a variable matrix. The 2 columns of the matrix consisted of the $\mathrm{x}$ and $\mathrm{y}$ axis location of the dot, and the rows of the matrix were the frame for where each respective movement occurred. These variables were then altered to produce a "delta" pixel movement by initializing the wind off pixel location as zero pixels. This allowed for each dot being tracked in Matlab to be viewed as delta $\mathrm{x}$ and y pixels moved during the test 


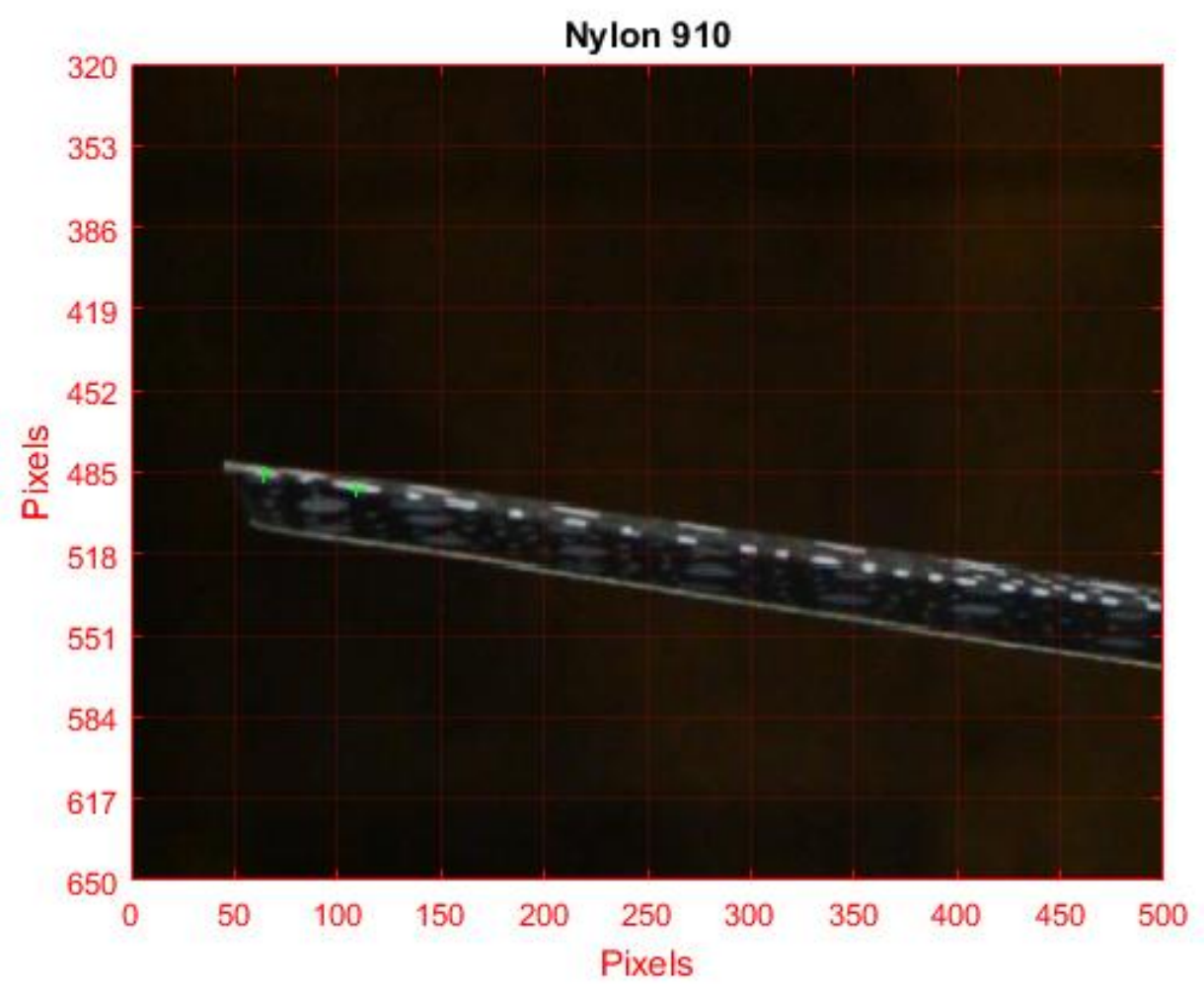

Figure 5.6: Dots being tracked by the x-axis camera

rather than absolute pixel location on the screen, making comparison to FEA much simpler and more robust once the data has been converted to pixels per centimeter. An example of this dot tracking can be seen in Figure 5.7.

In order to convert the pixels to total deflection during the experiment, a measurement between 3 dots on the Nylon 618 wing at the wingtip was taken. This measurement was then compared to the y-axis camera pixel location on a 1920x1080 pixel screen. Assuming primarily planar movement by the wingtip in the $\mathrm{z}$ and $\mathrm{x}$ direction, it was found that the $\mathrm{y}$-axis camera measured deflection at 5.2 pixels $/ \mathrm{mm}$. The same method was done for the $\mathrm{x}$-axis camera, but only for two dots near the wingtip. Because of the sweep of the wing, planar movement could not be assumed for dots that were far away from each other. It was found that at the wingtip, the $\mathrm{x}$-axis camera tracks the dots at approximately 3.6711 pixels/mm. 


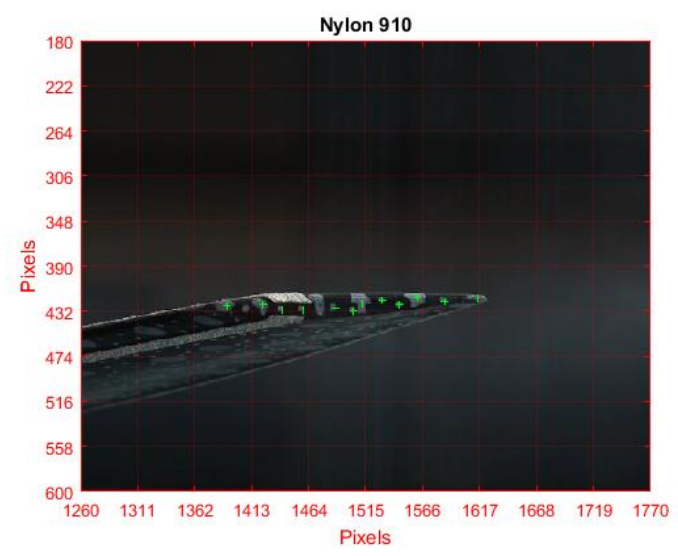

(a) Nylon 910 at 0 degrees, wind off

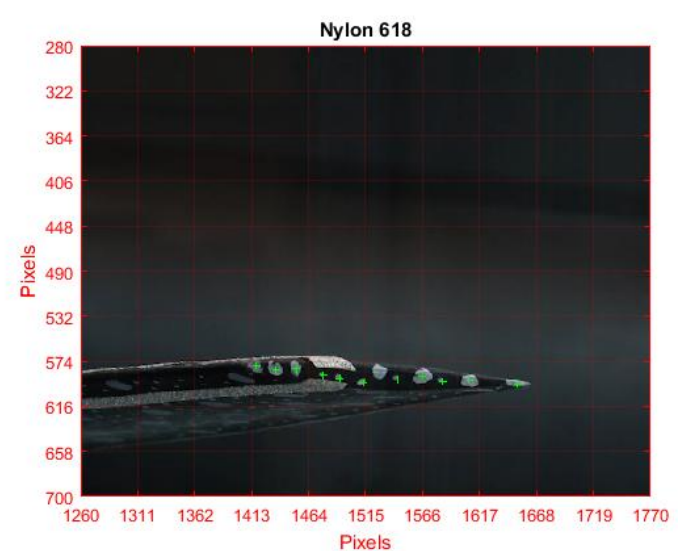

(c) Nylon 618 at 5 degrees, wind off

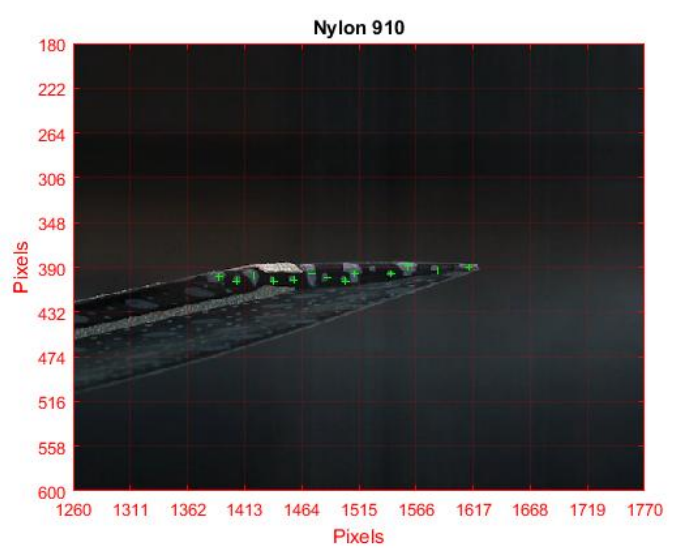

(b) Nylon 910, wind on

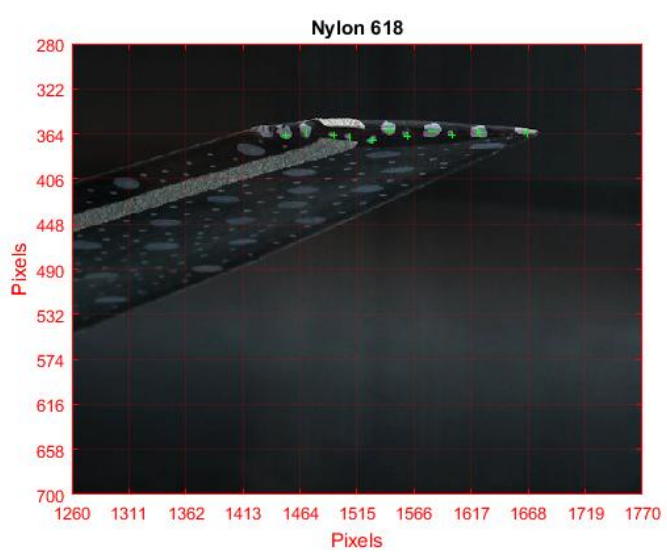

(d) Nylon 618, wind on

Figure 5.7: Screen captures of the dot tracking in Matlab during processing 


\section{DATA ANALYSIS}

\subsection{Aerodynamics}

Wake rake data taken from the Cal Poly Low Speed Wind Tunnel was first put through a calibration matrix to determine the magnitude of the dynamic pressure in the wake as well as the angle of that dynamic pressure. The calibration matrix uses data from the total port and 4 static ports in an evolutionary algorithm to resolve the pressure distributions. A Matlab function was created to upload the guesses and pressure readings from the rake, and download the resolved dynamic pressure and angles. These readings were then plotted as the ratio of the local total pressure to the wind tunnel total pressure, as well as the $\mathrm{Z}$ location in the wind tunnel in millimeters from centerline. The experimental wake distributions for both Nylon wings at all configurations can be seen in Figure 6.1. CFD data at these configurations was uploaded into Fieldview, and then plotted at identical locations as the Cal Poly wake rake for comparison.

Looking at Figure 6.1, we can see a clear shift in the $\mathrm{Z}$ locations of the wake for both wings when compared to CFD. Because the wing used in the wind tunnel can deflect, the peak pressure drop location in the wind tunnel will change with relation to this deflection, which is not possible in CFD without a 2-way Fluid Structure Interaction. This would also be the case for a wind tunnel model whose wing is made of a high strength material.

Another interesting metric when comparing the CFD data to the elastic wings is how the trends differ from 0 to 5 degrees angle of attack. Looking at Figure 6.1, we can see that the peak pressure drop in CFD at 0 degrees angle of attack is consistently less than both elastic wings. However, this trend reverses at 5 degrees angle of attack, and is accompanied by a major shift in the location of the wake for the spanwise locations. This is likely due to 

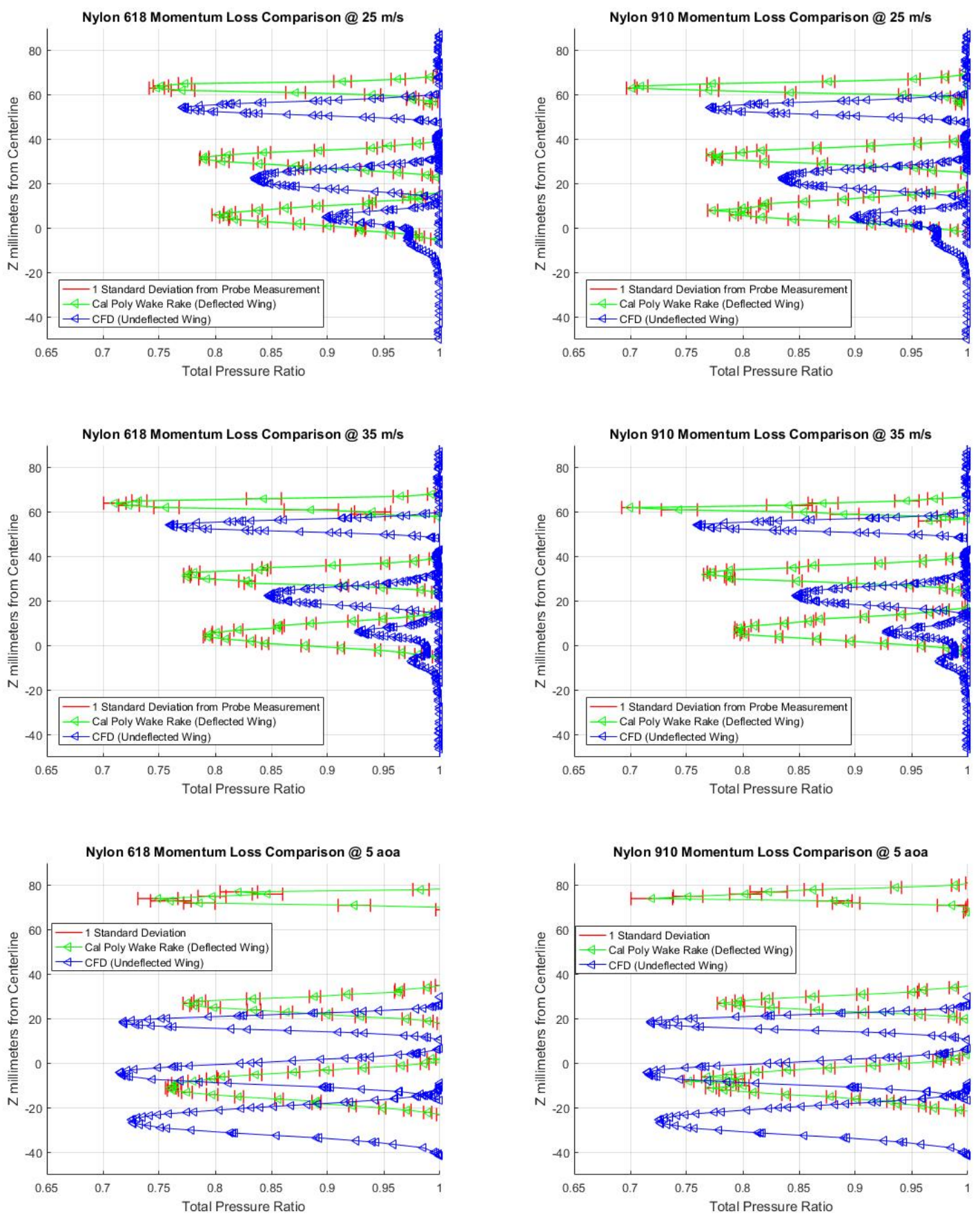

Figure 6.1: Total pressure ratios and their vertical locations for all wings and configurations from Cal Poly and CFD

the elastic wings producing less pressure drag because of deflection. The elastic wings have the ability to be moved in the flow, thus allowing the fluid additional avenues of movement away from the stagnation point, which is not present in the rigid wind tunnel model case 
shown by CFD. This change in the wake further displays how the elastic wing now behaves more like an aircraft in flight when compared to the rigid model exhibited in CFD.

Figure 6.2 gives an example of the pressure peak movement between CFD and the elastic wings for the 3 spanwise locations. Table 6.1 gives the delta values of the pressure peaks in the Cal Poly wind tunnel from CFD for each configuration tested. The addition and subraction metrics in Table 6.1 indicate the potential error of the measurement. An algorithm was produced to measure whether or not the peak location minus the standard deviation of the probe measurement was less than any adjacent location measurement plus the standard deviation at that point. If this was true for any of the peak locations, this meant that a false peak may have been measured, and needed to be recorded as potential error. Because the traverse can only move in $1 \mathrm{~mm}$ minimum increments, the error is determined to be accurate to within a millimeter.

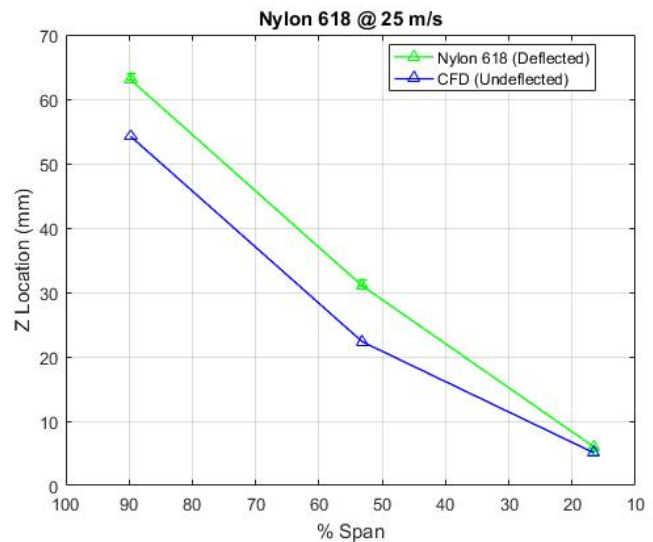

(a) Nylon 618 at $25 \mathrm{~m} / \mathrm{s}$

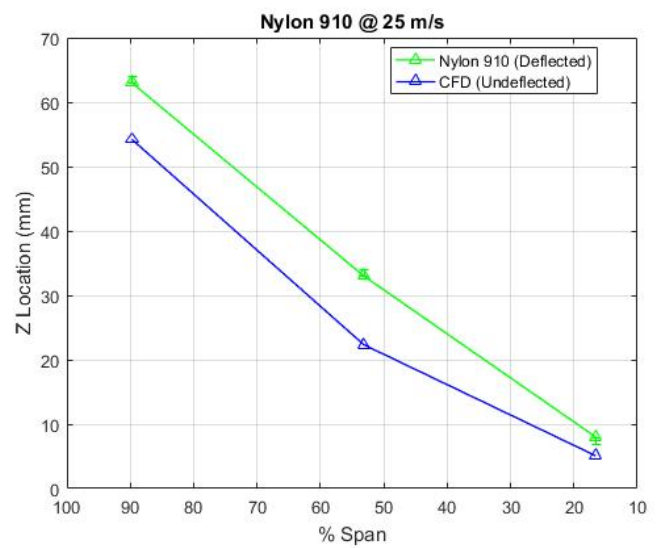

(b) Nylon 910 at $25 \mathrm{~m} / \mathrm{s}$

Figure 6.2: Pressure drop peak locations from tunnel centerline for both wings at $25 \mathrm{~m} / \mathrm{s}$

Table 6.1: Change in $\mathrm{Z}$ location of wake peaks for each configuration when compared to CFD

\begin{tabular}{|c|c|c|c|c|c|c|c|}
\cline { 3 - 8 } \multicolumn{1}{c|}{} & \multicolumn{3}{c|}{ Nylon 618 } & \multicolumn{3}{c|}{ Nylon 910 } \\
\cline { 4 - 8 } \multicolumn{1}{c|}{} & \% Span & 89.75 & 53.16 & 16.57 & 89.75 & 53.16 & 16.57 \\
\hline $25 \mathrm{~m} / \mathrm{s}$ & \multirow{3}{*}{$\Delta 2(\mathrm{~mm})$} & $8.8+1$ & $8.7+1$ & $0.9-1$ & $8.8+1$ & $10.7-2$ & $2.9 \pm 0$ \\
\cline { 4 - 8 } & $9.8-1$ & $8.7 \pm 1$ & $-1.9+1$ & $7.8 \pm 0$ & $9.7+1$ & $0.1 \pm 1$ \\
\hline & $55.2-1$ & $28.9-1$ & $15.6 \pm 1$ & $55.2 \pm 0$ & $28.9 \pm 0$ & $18.57 \pm 0$ \\
\hline
\end{tabular}


Looking at Table 6.1, it is interesting to see the similarity in the peak pressure drop location between the two wings tested. The Nylon 910 wing has less deflection than the Nylon 618 wing, which is discussed in more detail in the next section. However, we see from the table that the locations of their maximum pressure drop from the CFD simulations is relatively the same. This suggests that the flow angle is altered on both wings, with the 618 wing having more of a downwash component than the 910 wing. Furthermore, we see in Figure 6.3 that the wing wakes for both the 618 and 910 wings have very similar wake thickness distributions as well as maximum pressure drop values. This suggests that the wings have very similar drag forces at the spanwise locations examined, regardless of the amount of deflection each wing exhibits.

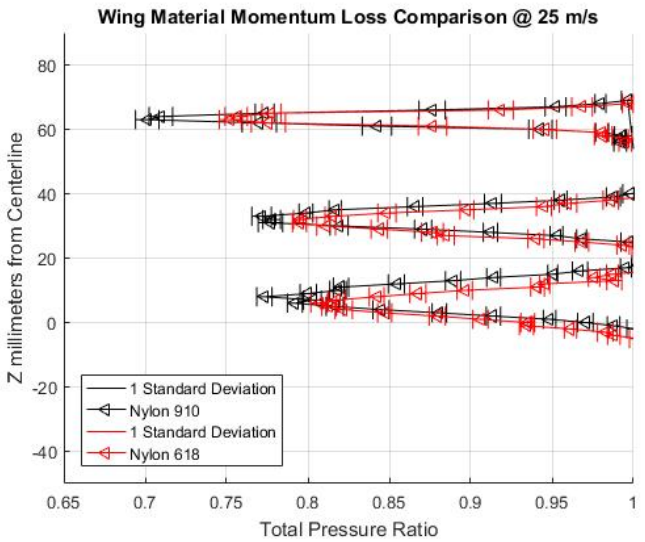

(a) Wake probe data comparison of both wings at 25 $\mathrm{m} / \mathrm{s}$

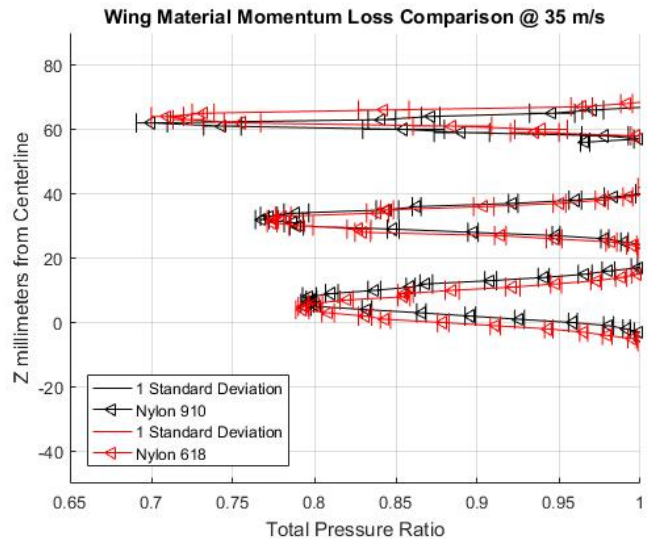

(b) Wake probe data comparison of both wings at 35 $\mathrm{m} / \mathrm{s}$

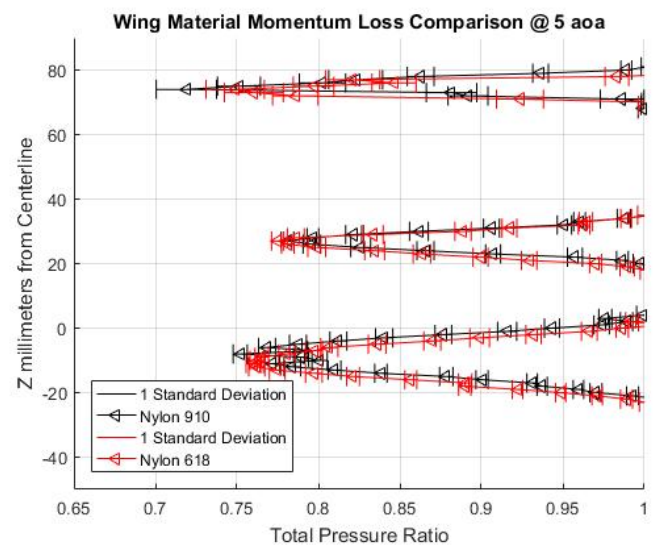

(c) Wake probe data comparison of both wings at 5 degrees angle of attack

Figure 6.3: Wing wake comparison from the Cal Poly wake rake probe 


\subsection{Structures}

Deflection data taken from the videogrammetry experiment was plotted in Matlab to better visualize the wingtip deflection. The plotting algorithm first identifies the dots on the wing that were successfully tracked for the entire experiment, as well as identifying the datum dots. If a dot became invalid during tracking, it was discarded. The algorithm then selects the valid dot that is closest to the trailing edge for plotting and analysis. The result of this data reduction is shown in Figure 6.4.

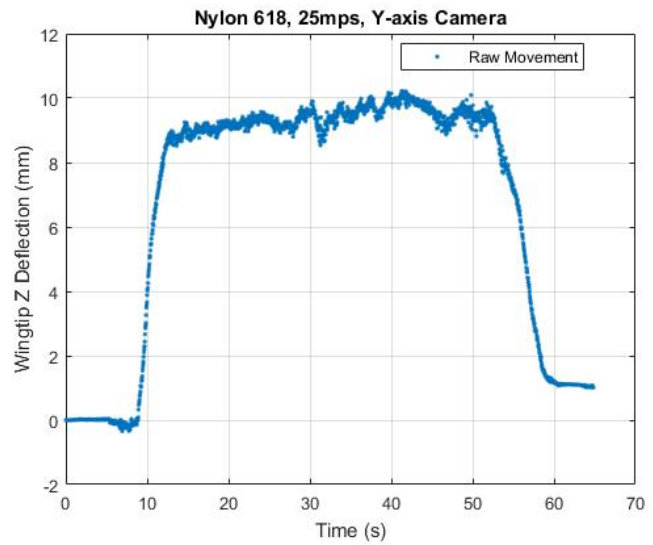

(a) $\mathrm{Z}$ axis deflection raw data

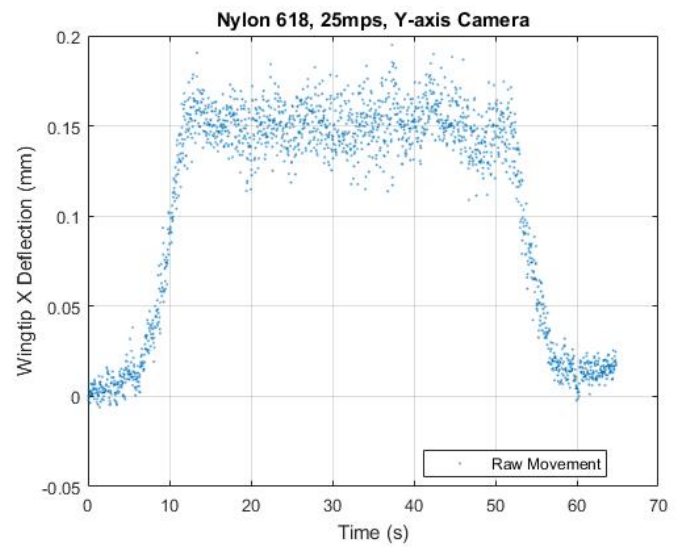

(b) $\mathrm{X}$ axis deflection raw data

Figure 6.4: Raw data from y axis camera on the Nylon 618 wing at $25 \mathrm{~m} / \mathrm{s}$

While it was useful to visualize the deflection over the duration of the experiment, it was not suitable to find an average deflection of the wing. Further data reduction was performed by placing time limits on "good" data. By looking at Figure 6.4, it was clear that the last 10 seconds could be omitted from analysis, since the tunnel was either in descent or beginning it. This was also the case for the start of the tunnel. However, because the Cal Poly wind tunnel assumes approximately 20 to 30 seconds for flow to properly settle in the test section, the first 30 seconds were also omitted from all data analysis. The observable region for wing deflection was narrowed down to the limits shown in Figure 6.5 


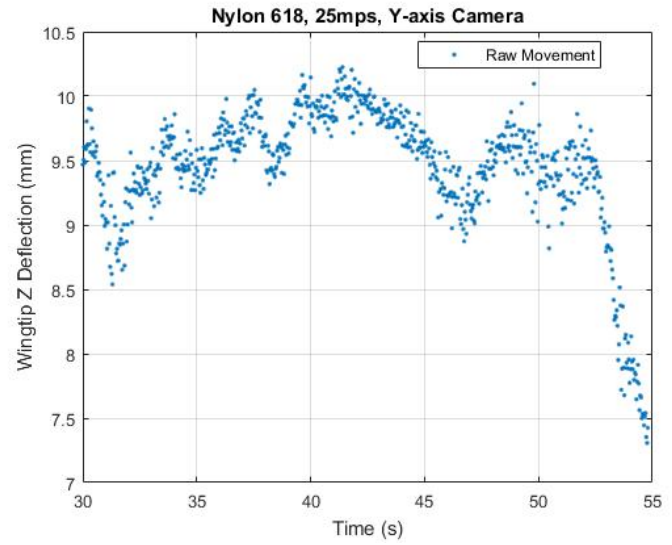

(a) $\mathrm{Z}$ axis deflection clipped data

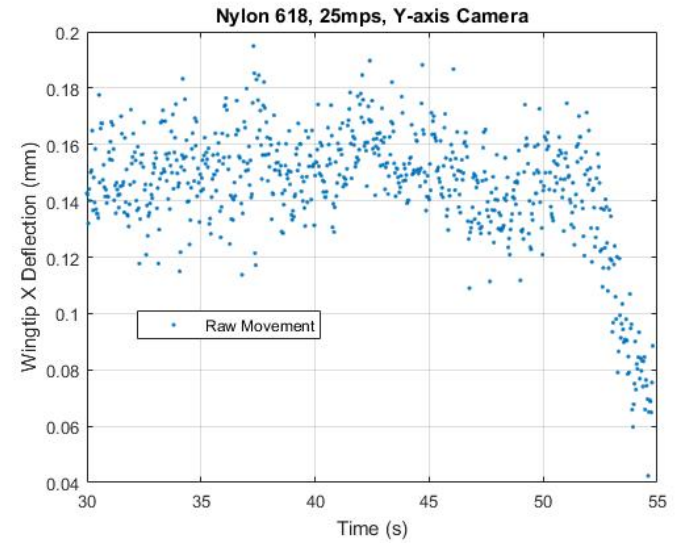

(b) $\mathrm{X}$ axis deflection clipped data

Figure 6.5: Clipped data from y axis camera on the Nylon 618 wing at $25 \mathrm{~m} / \mathrm{s}$

Within this range, it was desired to find the data that best represented the mean deflection of the wing with as little vibration as possible. This lead to the development of another data algorithm that sectioned the remaining data into 5 second intervals. Each 5 second interval was evaluated for the mean and standard deviation of the deflection measured by the cameras. The 5 second interval with the lowest standard deviation was interpreted to represent the wing deflection with minimal vibrations or noise presence. The mean of this 5 second interval was taken to be the wing deflection for that configuration, and the result of this algorithm for one configuration is shown in Figure 6.6

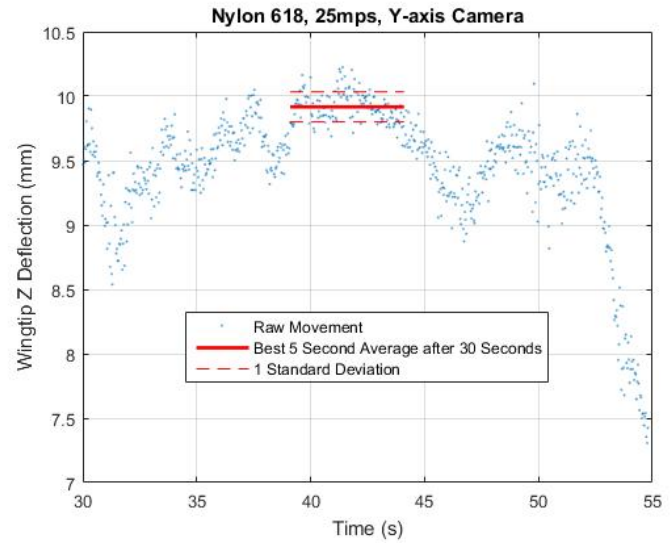

(a) $\mathrm{Z}$ axis deflection average

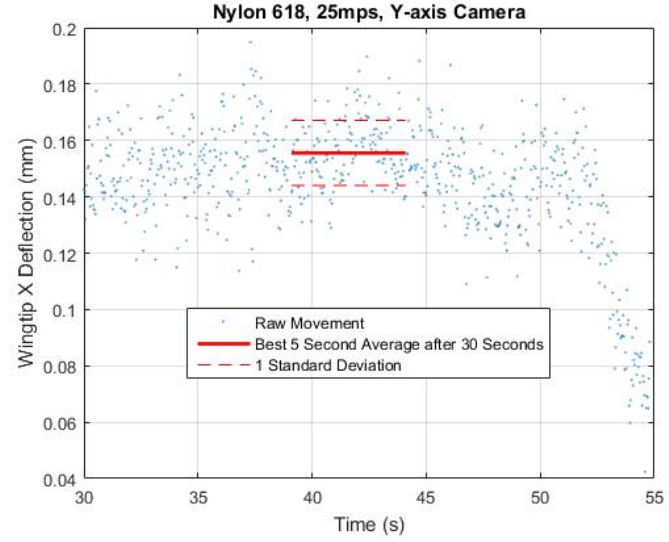

(b) $\mathrm{X}$ axis deflection average

Figure 6.6: Average data from y axis camera on the Nylon 618 wing at $25 \mathrm{~m} / \mathrm{s}$ 
With the algorithm in place, the data could be analyzed for each configuration for the $\mathrm{x}$ and $\mathrm{y}$ axis cameras. The $\mathrm{y}$-axis camera served as the primary data collector, while the $\mathrm{x}$-axis camera offered some validation to what was analyzed by the $\mathrm{y}$-axis camera in most cases. However, the $\mathrm{x}$-axis camera was not able to keep dot tracking validation for all 35 $\mathrm{m} / \mathrm{s}$ configurations, and therefore is not included. The videogrammetry deflection results are shown in Table 6.2.

Table 6.2: Deflection results from the videogrammetry experiment performed in the Cal Poly Low Speed Wind Tunnel

\begin{tabular}{|l|l|c|l|l|c|c|l|}
\cline { 3 - 8 } \multicolumn{2}{c|}{} & \multicolumn{3}{c|}{ Nylon 618 } & \multicolumn{3}{c|}{ Nylon 910 } \\
\cline { 3 - 9 } \multicolumn{2}{c|}{} & $25 \mathrm{~m} / \mathrm{s}$ & $35 \mathrm{~m} / \mathrm{s}$ & 5 AoA & $25 \mathrm{~m} / \mathrm{s}$ & $35 \mathrm{~m} / \mathrm{s}$ & 5 AoA \\
\hline \multirow{2}{*}{ Y-Camera } & Z deflection $(\mathrm{mm})$ & 9.9 & 14.0 & 45.3 & 5.9 & 7.29 & 37.1 \\
\cline { 2 - 8 } & X deflection $(\mathrm{mm})$ & 0.2 & 0.8 & 3.1 & 0.3 & 0.7 & 2.3 \\
\hline \multirow{2}{*}{ X-Camera } & Z deflection $(\mathrm{mm})$ & 10.3 & - & 48.8 & 5.3 & - & 34.3 \\
\cline { 2 - 8 } & Y deflection $(\mathrm{mm})$ & -1.3 & - & -8.0 & -0.9 & - & -6.7 \\
\hline
\end{tabular}

Looking at the results in Table 6.2, we see the trends expected for each wing. Deflection increases with higher speed, and even more so with higher angle of attack, because of the increase in lift associated with the flow regime and configuration. Furthermore, the Nylon 618 has more deflection in in the $\mathrm{Z}$ axis due to the material's mechanical properties being more flexible than Nylon 910.

Using the values in Table 6.2, a comparison was done to the results analyzed in FEA. While the assumptions made for the simulations hindered the accuracy of the outcome, it was still within a range that makes it useful for determining an area one would expect the wing to deflect to using a factor of safety. Because the videogrammetry data is primarily concerned with the wingtip trailing edge $\mathrm{Z}$-axis deflection, a probe was placed in this area in the simulation such that the point's movement could be tracked and logged. Figure 6.7 shows the deformed and undeformed model, as well as the point being tracked.

Using the probe location in Figure 6.7, z-axis deflection was tracked from the end of the application of a gravity load until the end of the application of the aerodynamic load. 


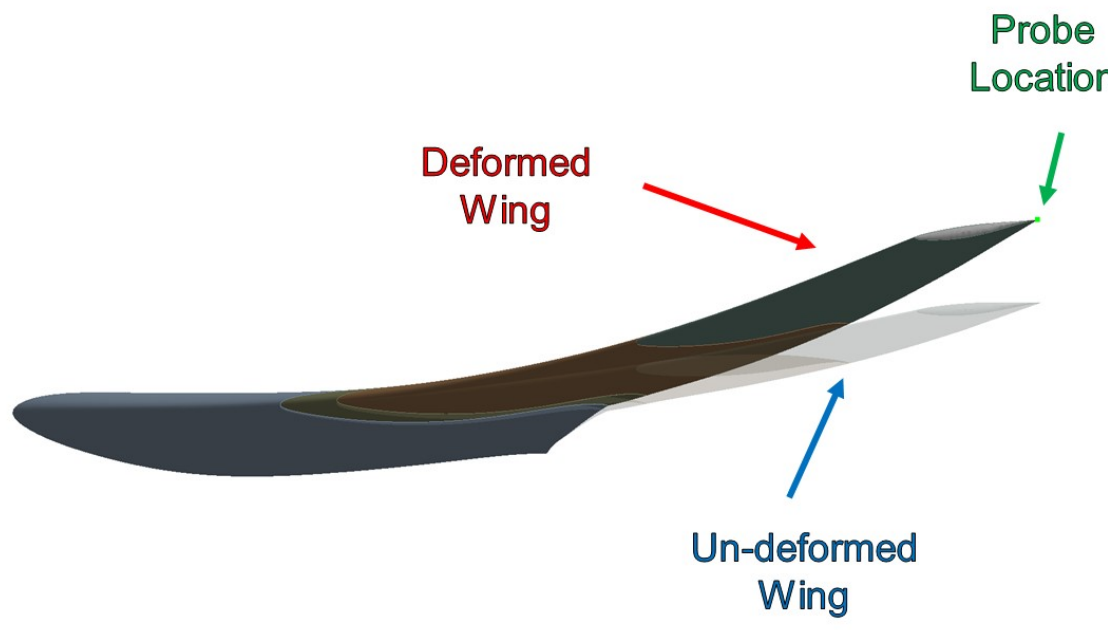

Figure 6.7: Undeformed and deformed wing result from FEA, with indication of probe placement

Once the simulation was completed, the change in $\mathrm{z}$ deflection was calculated for each wing material and each configuration. The results were then compared to the results from videogrammetry, and are displayed in Table 6.3

Table 6.3: Results of the FEA simulations compared to the videogrammetry experiment

\begin{tabular}{|c|c|c|c|c|c|c|c|}
\cline { 3 - 8 } \multicolumn{2}{c|}{} & \multicolumn{3}{c|}{ Nylon 618} & \multicolumn{3}{c|}{ Nylon 910} \\
\cline { 3 - 8 } \multicolumn{2}{c|}{} & $25 \mathrm{~m} / \mathrm{s}$ & $35 \mathrm{~m} / \mathrm{s}$ & 5 AoA & $25 \mathrm{~m} / \mathrm{s}$ & $35 \mathrm{~m} / \mathrm{s}$ & 5 AoA \\
\hline \multirow{3}{*}{$\Delta Z(\mathrm{~mm})$} & FEA & 9.0 & 20.6 & 41.3 & 4.8 & 10.9 & 21.8 \\
\cline { 2 - 8 } & Videogrammetry & 9.9 & 14.0 & 45.3 & 5.9 & 7.3 & 37.1 \\
\cline { 2 - 8 } & \% Error & 9.1 & 47.0 & 8.8 & 18.6 & 49.3 & 41.2 \\
\hline
\end{tabular}

Table 6.3 indicates that all deflection measurements are within a factor of safety of 3 . However, many of the results from FEA are excessive, and are likely due to the assumptions made during the simulation. There are various reasons for the error present in Table 6.3.

1. 1-Way Simulation - The solution accuracy would undoubtedly improve with the implementation of a 2-way fluid structure interaction. While requiring additional settings in Ansys and computer resources, it is a more accurate representation of the testing phenomena quantified in the experiment. 
2. Material Property Assumptions - Not only does FDM material behave as an anisotropic material rather than an isotropic, it needs to be tested by the printer it was made from, as there are large amounts of manufacturing differences available from $3 \mathrm{D}$ printers. A more in depth quantification of the material properties of the printed parts and additional modeling in FEA of those anisotropic properties would reduce the error significantly. However, this was not performed for this study due to a lack of available resources as well as being outside the scope of the study.

3. Internal Structure - The parts made via FDM are usually accompanied by an internal structure rather than a filled volume part, in this case the structure was honeycomb. The structure is likely altering the movement of the parts under pressure load, and is something that is difficult to model in CAD for analysis in FEA.

4. Manufacturing Error - The FEA uses the exact dimensions imported from CAD, while the $3 \mathrm{D}$ printer can introduce some manufacturing error to the part in terms of warping, splitting, and cavitation. While numerous practice parts were made in attempts to resolve these manufacturing errors, these inconsistencies were still present in all the parts produced, and can cause various structural changes that are not present in the simulation. 


\section{CONCLUSION}

The data presented gives indication that producing structurally and aerodynamically representative wind tunnel models is feasible for research oriented experiments, specifically in low Reynolds and small scale applications. The ability to produce the models in relatively short turnaround time is invaluable when considering the cost and time required to produce high strength metal wind tunnel models and jigs. Furthermore, the advantages of additive manufacturing allow the user to tailor the interior of the wind tunnel model to not only meet structural deflection requirements, but also constraints presented by internal wiring, tubing, and mechanisms that are typically present in wind tunnel models. However, it is certainly noted that the repeat ability of these results, and for FDM models at this scale, is subjective to the manufacturing process.

In regards to comparison between wind tunnel and CFD data for elastic wings, it is clear that a non-deforming CFD shape lacks the ability to encapsulate the aerodynamic changes due to wing deflection. While further investigation is necessary to determine if a 2 way Fluid-structure-interaction can better represent the data seen in the wind tunnel experiment, it is clear from the experimental data that the CFD information for a 1 way simulation cannot produce the accurate data of a deflected wing at low Reynolds.

The results garnered from FEA compared to videogrammetry represent some discrepancies. While the assumptions made in the FEA simulations were necessary, these assumptions can be mitigated through multiple techniques and a higher fidelity simulation. However, this can be counter productive in that it can drastically increase the time and effort necessary to get a truly accurate representation of the deflection due to aerodynamic loads. From a research standpoint, the amount of time necessary to solve the FEA simulations for this 
project were much more advantageous than accuracy within 5\%, and will likely be the case for further research related projects.

\subsection{Future Work Considerations}

\subsubsection{Experimental}

In order to produce more flight accurate wind tunnel models, additional testing is necessary to validate the data and claims made in this project. First and foremost being that the experiment requires a rigid metal wind tunnel model for comparison. This would not only act as an independent variable in the experiment, but would also serve as an excellent CFD validation tool. There are also many other experiments involving the elastic wings that can build on the data presented within.

1. Test Additional Reynolds Numbers - While this is due to the constraints present in the Cal Poly wind tunnel, this information is invaluable to see the effects deflection has with Reynolds number. This could then be validated with additional CFD cases, or better, with flight test data of a representative aircraft.

2. Force Data - Data from a force balance could serve as a validation tool to the deflected measurements and drag data for the wings.

3. Surface Pressure Measurements - Pressure taps along the surface of the wing would allow the acquisition of lift distribution change along the wing as it deflects. This could ultimately validate the deflections seen during the experiment.

4. Manufacturing Process - Ideally, the wings produced would be made as a single part, rather than 3 to increase the fidelity of the FEA simulations. A smaller scale 
model or a larger printer could improve the accuracy of both the experiment and the simulations.

5. Structural Testing - Detailed mechanical properties from 3D printed parts would aid in the ability to predict their deformation in FEA. While information about the materials for this project was obtained from the manufacturer data, it does not address the additional variables present in 3D printing, such as infill, layer width, and other settings during the manufacturing process. Producing tensile test specimens that investigate how the mechanical properties change with these settings would improve the fidelity of the results.

6. Dynamic Testing - A high speed camera could be utilized to track the dots along the wing to catch the vibration frequency of the wings. This could be very valuable in determining why the static wing deflection behaves as it does.

\subsubsection{Numerical}

Further investigations into the numerical simulations would determine not only the ability to predict the change in the model shape more accurately prior to construction, but could also serve as additional validation to the information obtained during the experiment. However, due to the complexity of accurately simulating 3D printed parts in FEA, assumptions will likely still need to be made in order to solve the simulations appropriately.

1. 2 way Fluid-Structure-Interaction - While still using the assumptions made in FEA, this type of simulation would be able to indicate to the user whether or not the simulation's percent difference to the experimental data could be mitigated. While these simulations are normally done to produce the jig models for wind tunnel experiments, the complexity increases with 3D printed parts as well as elastic parts, due to the elastic part's ability to oscillate more than rigid parts. 
2. Anisotropic FEA Model - Because parts manufactured by a 3D printer represent a homogenous anisotropic material, the FEA simulations should reflect this. While not ideal for this research project due to the time and scope increase it would cause, it could still be done for future experiments and would likely produce more accurate results. However, additional advancements in the regime of $3 \mathrm{D}$ printed parts and FEA may be necessary before it is even possible.

3. 3D Printing Alteration - An interesting investigation would be to see the feasibility of altering the solid model to produce the deflection present in FEA using the results from FEA in the slicing algorithm that the 3D printer uses. This could produce an entirely new algorithm that produces interior 3D printed part structure tailored to wind tunnel models that need to meet deflection and internal cavity requirements.

4. Vibration Mode in FEA - Similar to testing for the vibrations on the wing in the experiment, it could also be tested in FEA for correlation and to garner further information about the model prior to testing. 


\section{BIBLIOGRAPHY}

[1] ASTM. "Standard test method for tensile properties of plastics". In: ASTM International. Designation: D 08 (2003). uRL: http://scholar.google.com/scholar?hl=en\% 7B\%5C\&\%7DbtnG=Search\%7B\%5C\&\%7Dq=intitle:Standard+Test+Method+for+ Tensile+Properties+of+Plastics $\% 7 \mathrm{~B} \% 5 \mathrm{C \# \%}$ 7D0.

[2] ASTM International. “ASTM D 790 - 02 - Flexural Properties of Unreinforced and Reinforced Plastics and Electrical Insulating Materials”. In: 14 (2002), pp. 146-154. DOI: 10.1520/D0790-10..

[3] N Bier, S Keye, and D Rohlmann. "Design of a Wind Tunnel Model for Maximum Lift Predictions Based on Flight Test Data”. In: (2013), pp. 1-10. Dor: doi:10.2514/ 6.2013-2930.

[4] Albert L. Braslow and Eugene C. Knox. "Simplified Method for Determination of Critical Height of Distributed Roughness Particles for Boundary-layer Transition at Mach Numbers from 0 to 5”. In: NACA Technical Note 4363 (1958).

[5] Jochen Carl, Dana Muller-Hoeppe, and Morgan Meadows. "Comparison of Tetrahedral and Brick Elements for Linear Elastic Analysis". In: (2006).

[6] Ubaldo Cella and Marco Evangelos Biancolini. "Aeroelastic Analysis of Aircraft Wind-Tunnel Model Coupling Structural and Fluid Dynamic Codes”. In: Journal of Aircraft 49 (2012), pp. 407-414. ISSN: 0021-8669. DOI: 10.2514/1.C031293. URL: http://arc.aiaa.org/doi/abs/10.2514/1.C031293.

[7] Raymond N Chuk and Vincent $\mathbf{J}$ Thomson. "A comparison of rapid prototyping techniques used for wind tunnel model fabrication”. In: Rapid Prototyping Journal 4.4 (2016), pp. 185-196. 
[8] S Daneshmand, A Ahmadi Nadooshan, and C Aghanajafi. "Investigation of Layer Thickness and Surface Roughness on Aerodynamic Coefficients of Wind Tunnel RP Models". In: Engineering and Technology 26 (2007), pp. 7-12.

[9] JR Hooker, AW Burner, and R Valla. "Static aeroelastic analysis of transonic wind tunnel models using finite element methods". In: AIAA Paper (1997). URL: http: //arc.aiaa.org/doi/pdf/10.2514/6.1997-2243.

[10] Stefan Keye. "Fluid-Structure Coupled Analysis of a Transport Aircraft and FlightTest Validation". In: Journal of Aircraft 48 (2011), pp. 381-390. ISSN: 0021-8669. DOI: 10.2514/1.C000235.

[11] Kurt Long. Transition Issues for ITD-51A Tests. Tech. rep. 2014.

[12] MatWeb. Nylon 618 Mechanical Properties. http://www. matweb.com/search/ DataSheet.aspx?MatGUID=726845c457b94b7cafe31d2e65739e1d\&ckck=1.

[13] Nhan T Nguyen, Eric Ting, and Sonia Lebofsky. "Aeroelastic Analysis of Wind Tunnel Test Data of a Flexible Wing with a Variable Camber Continuous Trailing Edge Flap (VCCTEF)". In: 56th AIAA/ASCE/AHS/ASC Structures, Structural Dynamics, and Materials Conference (2015), pp. 1-28. DOI: doi:10.2514/6.2015-1405. URL: http://dx.doi.org/10.2514/6.2015-1405.

[14] NinjaTek. Nylon 910 ALLOY-TDS. Tech. rep. 2016, p. 1.

[15] Andrei V. Popov et al. "Real Time Morphing Wing Optimization Validation Using Wind-Tunnel Tests". In: Journal of Aircraft 47 (2010), pp. 1346-1355. Issn: 00218669. DOI: $10.2514 / 1.47431$.

[16] Melissa B. Rivers and Ashley Dittberner. "Experimental Investigations of the NASA Common Research Model”. In: Journal of Aircraft 51 (2014), pp. 1183-1193. IsSN: 0021-8669. DOI: 10.2514/1.C032626. URL: http://arc.aiaa.org/doi/abs/10.2514/1. C032626. 
[17] A.Y.N. Sofla et al. "Hierarchical modeling and optimization of camber morphing airfoil”. In: Aerospace Science and Technology 42 (2015), pp. 31-38. ISSN: 12709638. DOI: 10.1016/j.matdes.2009.09.011. URL: http://dx.doi.org/10.1016/j.matdes.2009. 09.011 .

[18] Eric Ting. "Static Aeroelastic Scaling and Analysis of a Sub-Scale Flexible Wing Wind Tunnel Model”. In: (2010), pp. 1-41. DoI: 10.2514/6.2014-0838.

[19] B. M. Tymrak, M. Kreiger, and J. M. Pearce. "Mechanical properties of components fabricated with open-source 3-D printers under realistic environmental conditions". In: Materials and Design 58 (2014), pp. 242-246. ISSN: 18734197. DOI: 10.1016/j. matdes.2014.02.038. uRL: http://dx.doi.org/10.1016/j.matdes.2014.02.038. 


\section{APPENDICES}

\section{A CAD DRAWINGS}

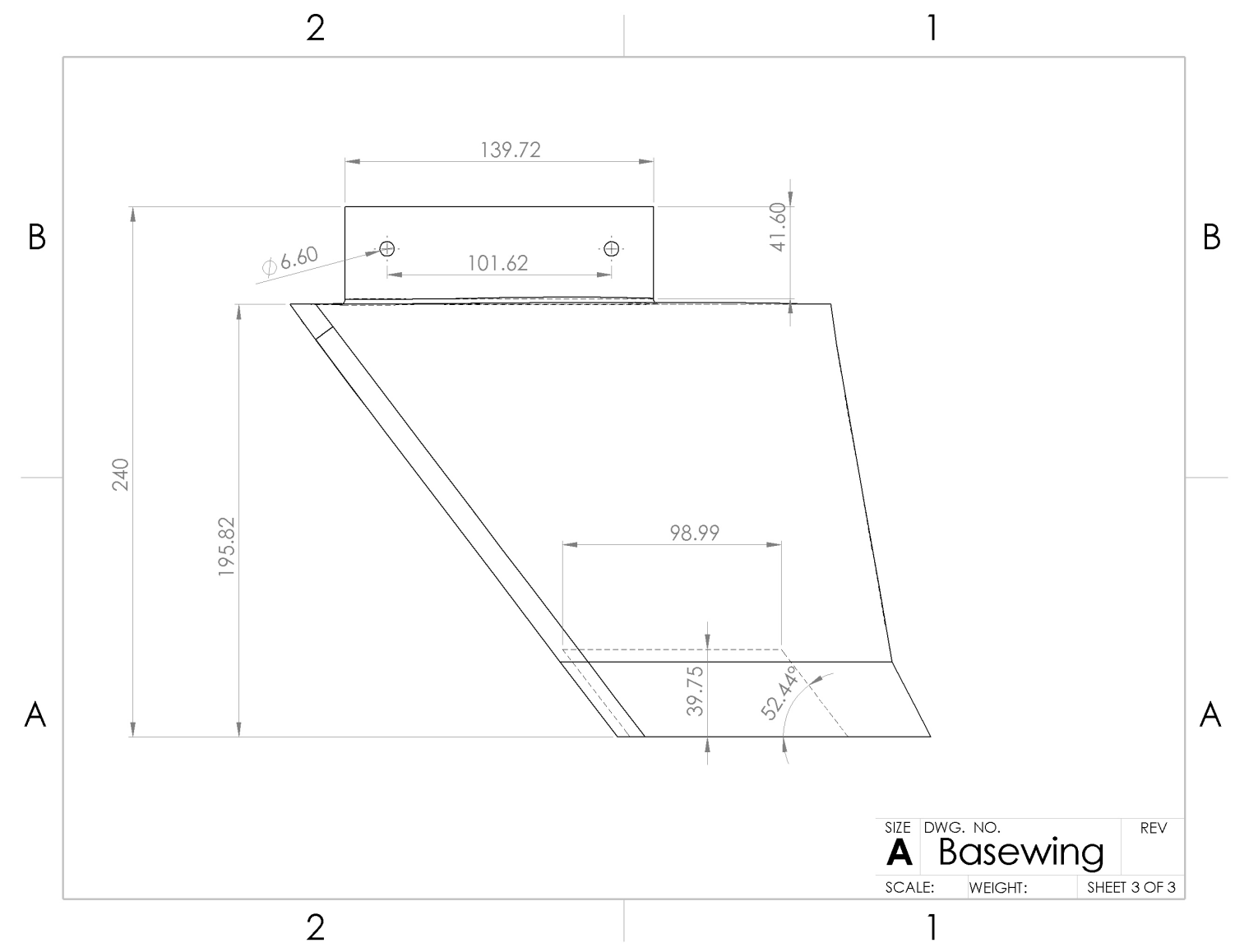

Figure A.1: Basewing dimensions 


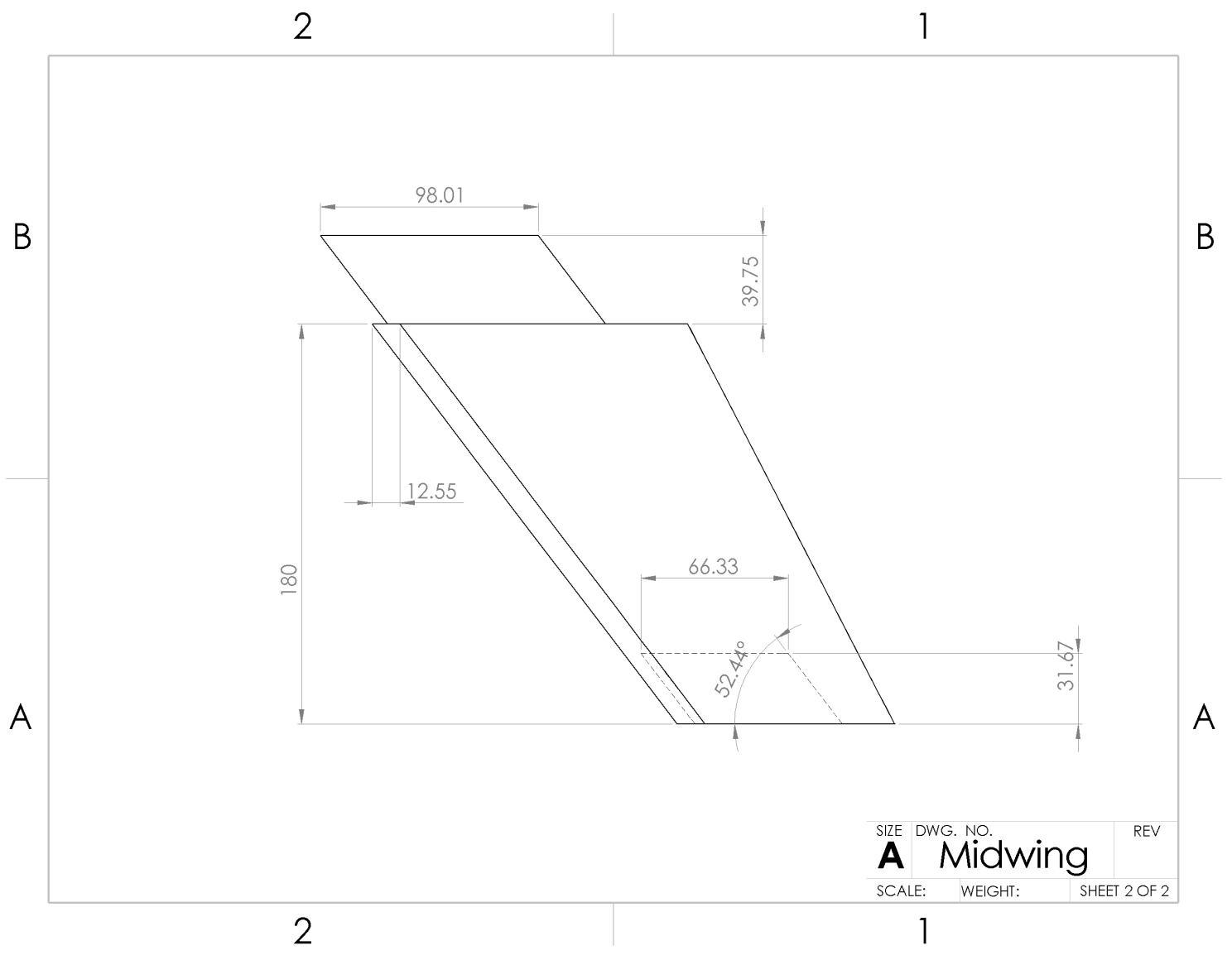

Figure A.2: Midwing dimensions 


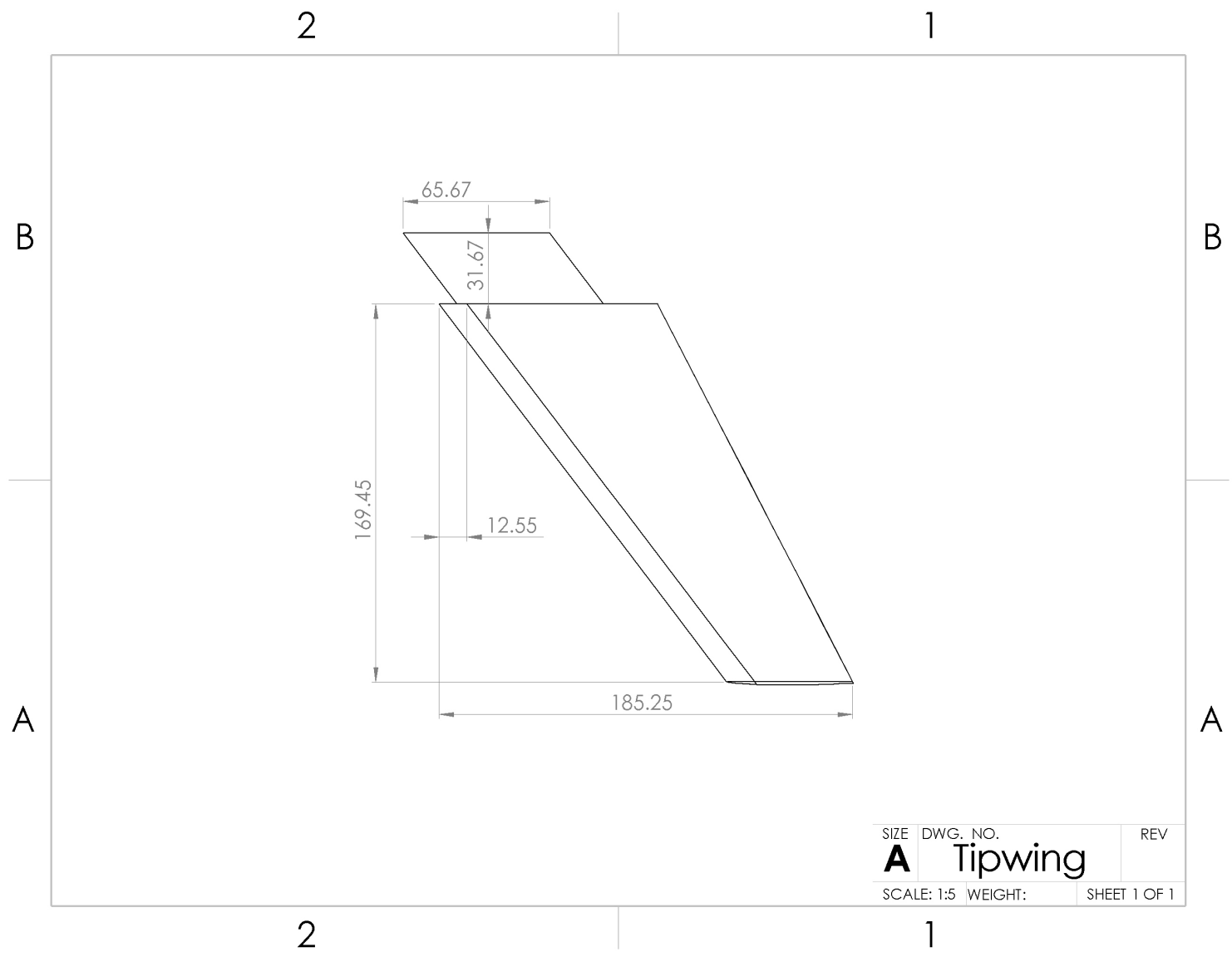

Figure A.3: Tipwing dimensions 


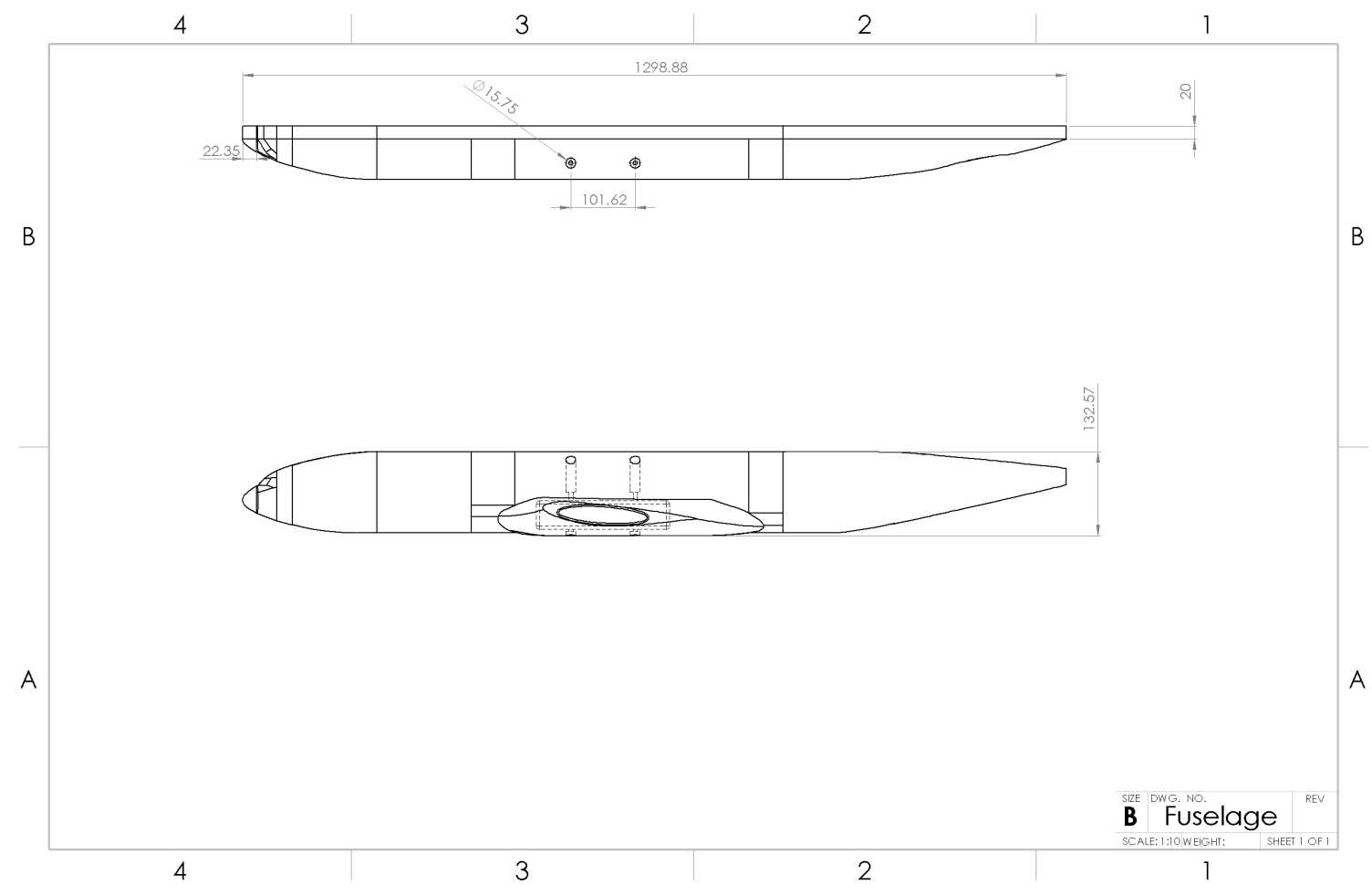

Figure A.4: Fuselage dimensions 


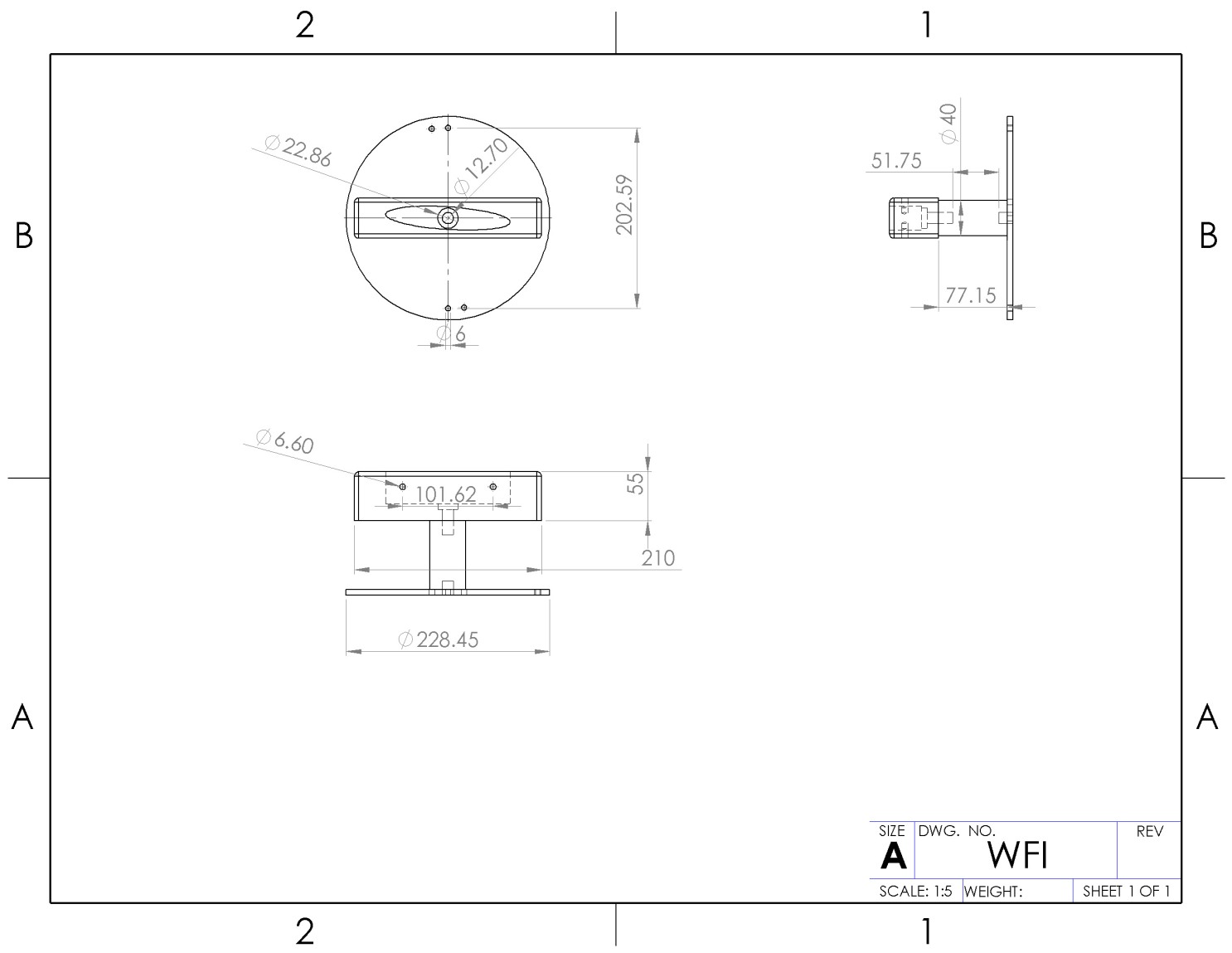

Figure A.5: Tunnel mounting assembly dimensions 


\section{B MESHING PARAMETERS}

Table B.1: Global meshing parameters for both angles of attack

\begin{tabular}{|c|c|}
\hline Setting & Selection \\
\hline Advanced Sizing Function & On: Curvature \\
\hline Relevance Center & Fine \\
\hline Smoothing & High \\
\hline Transition & Slow \\
\hline Span Angle Center & Fine \\
\hline Curvature Normal Angle & 5 degrees \\
\hline Min Size & $7.5 \mathrm{e}-004 \mathrm{~m}$ \\
\hline Max Face Size & $7 \mathrm{e}-002 \mathrm{~m}$ \\
\hline Max Size & $7 \mathrm{e}-002 \mathrm{~m}$ \\
\hline Triangle Surface Mesher & Advancing Front \\
\hline
\end{tabular}

Table B.2: Global meshing parameters for the FEA simulation

\begin{tabular}{|c|c|}
\hline Setting & Selection \\
\hline Physics Preference & Mechanical \\
\hline Relevance & 100 \\
\hline Advanced Sizing Function & On: Proximity and Curvature \\
\hline Relevance Center & Fine \\
\hline Smoothing & High \\
\hline Transition & Slow \\
\hline Span Angle Center & Fine \\
\hline Curvature Normal Angle & 1 degree \\
\hline Number Cells Across Gape & 10 \\
\hline Max Face Size & $1 \mathrm{e}-002 \mathrm{~m}$ \\
\hline Max Size & 5e-002 m \\
\hline Triangle Surface Mesher & Advancing Front \\
\hline
\end{tabular}

